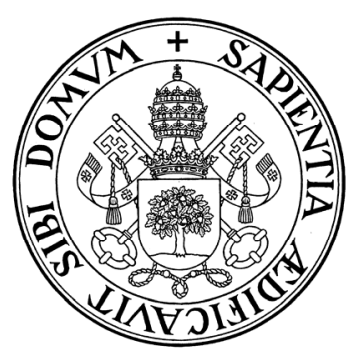

UNIVERSIDAD DE VALLADOLID

\title{
MÉTODOS NUMÉRICOS TIPO RUNGE-KUTTA-NYSTRÖM PARA LA INTEGRACIÓN EFICIENTE DE PROBLEMAS OSCILATORIOS
}

Amelia García Garrosa

Tesis doctoral

\author{
DEPARTAMENTO DE \\ MATEMÁTICA APLICADA A LA INGENIERÍA
}




\title{
UNIVERSIDAD DE VALLADOLID
}

\section{DEPARTAMENTO DE MATEMATICA APLICADA A LA INGENIERIA}

\author{
Tesis doctoral \\ Autor: Dña. Amelia García Garrosa \\ Director: Dr. D. Pablo Martín Ordóñez \\ Dra. Da. Ana Belén González Martínez \\ Título: Métodos numéricos tipo Runge-Kutta-Nyström para \\ la integración eficiente de problemas oscilatorios. \\ Tribunal \\ Presidente: Dr. D. José Manuel Ferrándiz Leal \\ Universidad de Alicante \\ Vocales: $\quad$ Dr. D. Juan Getino Fernández \\ Universidad de Valladolid \\ Dr. D. Antonio Vigueras Campuzano \\ Universidad Politécnica de Cartagena \\ Dr. D. Manuel Palacios Latasa \\ Universidad de Zaragoza \\ Secretario: Dr. D. José Miguel Farto Álvarez \\ Universidad de Valladolid
}

Fecha de lectura: 30 de noviembre de 2001.

Calificación: SOBRESALIENTE CUM LAUDE. 


\section{Contenido}

Agradecimientos $\quad$ iii

Introducción $\quad$ v

1 Métodos de un paso para la integración de ecuaciones $\begin{array}{lr}\text { de segundo orden } & 1\end{array}$

1.1 Introducción . . . . . . . . . . . . . . . . . . . . 1

1.2 Métodos Runge-Kutta-Nyström . . . . . . . . . . . . 2

1.3 Métodos RKGM . . . . . . . . . . . . . . . . 4

2 Nuevos métodos tipo Runge-Kutta-Nyström. 11

2.1 Introducción . . . . . . . . . . . . . . . 11

2.2 Nuevos métodos especiales para

problemas oscilatorios . . . . . . . . . . . 13

2.2.1 Discusión de métodos de orden oscilatorio cinco 20

2.2.2 Discusión de métodos de orden oscilatorio seis . 24

2.2.3 Experimentos numéricos . . . . . . . . . . 25

2.3 Métodos RKN $h^{2}$. Propiedades . . . . . . . . . . . 31

2.3.1 Formulación general . . . . . . . . . . 31

2.3.2 Error de truncación local . . . . . . . . . . 33

2.3.3 Condiciones de orden para un método

$\mathrm{RKN} h^{2} p: q \ldots \ldots . \ldots . \ldots 39$

2.4 Método RKNh $h^{2} 4: 5$ óptimo . . . . . . . . . . . . . . . 40

3 Métodos de paso variable 49

3.1 Introducción . . . . . . . . . . . . . . . . . . . . 49

3.2 Construcción de un par encajado

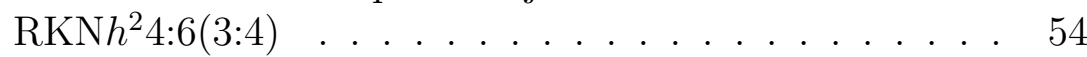

3.3 Experimentos numéricos . . . . . . . . . . . . 55 
4 Métodos $\mathrm{RKN} h^{2}$ de orden alto $\quad 69$

4.1 Introducción . . . . . . . . . . . . . . . . . 69

4.2 Teoría de árboles para los métodos $\mathrm{RKN} h^{2}$. . . . . . . 70

4.2.1 Árboles especiales de Nyström . . . . . . . . . . . 70

4.2.2 Derivadas de la función $f$. . . . . . . . . . . 72

4.2.3 Derivadas de los valores $k_{i} \ldots \ldots . . . . . .74$

4.2.4 Condiciones de orden. Error de truncación local 77

4.2.5 Árboles para un oscilador no perturbado. Condiciones de orden . . . . . . . . . . . . . . . 82

4.2.6 Condiciones de orden para métodos $\mathrm{RKN} h^{2} p: q \ldots \ldots \ldots . \ldots . \ldots . \ldots 85$

4.2.7 Condiciones simplificadoras . . . . . . . . . 87

4.3 Un método RKN $h^{2}$ de paso variable y orden 8 . . . . 96

4.3.1 Construcción del método de orden 8 . . . . . 96

4.3.2 Construcción del método de orden 6 . . . . . 103

4.3.3 Experimentos numéricos. . . . . . . . . . 108 


\section{Agradecimientos}

El presente trabajo ha sido realizado bajo la dirección de los profesores Pablo Martín Ordóñez y Ana Belén González Martínez, a quienes debo agradecer la propuesta del tema, su labor de dirección y su enorme paciencia y estímulo en todo momento.

También quiero citar a los profesores José Miguel Farto Álvarez y David Javier López Medina, tanto por sus comentarios y correcciones a lo largo del trabajo, como por la confianza que siempre depositaron en mí.

Quiero hacer constar que esta memoria ha sido realizada con la ayuda financiera de la Junta de Castilla y León bajo el proyecto VA11/99 y del Ministerio de Ciencia y Tecnología bajo el proyecto AYA2000-1787.

Finalmente, quiero manifestar mi más profundo agradecimiento a todas aquellas personas que con su constante aliento han hecho posible que esta memoria vea la luz. 


\section{Introducción}

En la segunda mitad del siglo pasado se han realizado numerosos trabajos sobre métodos numéricos para la integración de ecuaciones diferenciales ordinarias. En la actualidad, numerosos paquetes de software contienen rutinas de propósito general para la resolución de sistemas de ecuaciones diferenciales de primer orden. Dado que una ecuación diferencial de cualquier orden puede ser escrita como un sistema de ecuaciones de primer orden, dichas rutinas pueden utilizarse para resolver ecuaciones de cualquier orden. Esto podría hacer pensar que no es necesario el desarrollo de algoritmos específicos para tipos particulares de ecuaciones diferenciales, pero esta clase de estudios no es un trabajo inútil ya que al exigir menos generalidad podemos obtener métodos más eficientes.

Uno de los tipos que aparece a menudo en las ciencias aplicadas es la ecuación de segundo orden. Esto es debido, fundamentalmente, al hecho de que las fuerzas son proporcionales a las aceleraciones, es decir, a las derivadas segundas. Dentro de los algoritmos especialmente diseñados para ecuaciones de segundo orden están los de tipo multipaso como los métodos de Falkner [23], también llamados fórmulas de extrapolación de Adams para ecuaciones diferenciales de segundo orden [14] (véase también Martín et al. [85]), los métodos de Störmer-Cowell (véase Dormand [19] o Henrici [57]) o la reformulación de estos conocida como métodos de Gauss-Jackson (véase Dormand [19], González y Martín [46], Herrick [58], Jackson [64] o Merson [89]). Otro tipo de algoritmos, y posiblemente los más utilizados, para la integración numérica de ecuaciones de segundo orden son los métodos Runge-Kutta-Nyström (véase Dormand [19] o Hairer et al. [54]).

En esta memoria estamos interesados en un tipo más particular de problemas: los problemas oscilatorios. Esta clase de problemas aparece a menudo en el campo de la dinámica orbital. Como es bien 
sabido, la dinámica orbital estudia el movimiento de dos o más cuerpos que interactúan de acuerdo con la ley de Newton y que, posiblemente, están sujetos a la acción de otras fuerzas perturbadoras. Esto da lugar a una considerable variedad de comportamientos que pueden ser consultados en libros especializados como los de Szebehely [106] o Marchal [76]. De todos modos, en la práctica, los casos más interesantes son pequeñas perturbaciones de un problema puro de dos cuerpos. Las dificultades en este tipo de problemas vienen fundamentalmente de que en muchas ocasiones es necesario obtener soluciones muy precisas válidas durante un largo intervalo de tiempo y esto hace que los métodos de propósito general citados anteriormente, tanto para ecuaciones de primer orden como para ecuaciones de segundo orden, no sean adecuados.

Si las ecuaciones de Newton son integradas directamente con los métodos habituales, incluso en el caso de una partícula que describe un movimiento uniforme en una órbita circular, la solución numérica puede describir una espiral hacia el interior debido a la inestabilidad orbital (Lambert y Watson [70]), con lo que los resultados obtenidos se mantienen válidos por un periodo muy corto de tiempo. Para evitar este tipo de problemas se suelen utilizar dos tipos de técnicas. Una de ellas es, antes de realizar la integración numérica, transformar las ecuaciones del movimiento para eliminar el mal comportamiento antes mencionado (Ferrándiz [29], Ferrándiz y Martín [30], Janin [66], Stiefel y Scheifele [103], etc.). La otra técnica consiste en diseñar métodos numéricos especiales para reducir los errores y eliminar la inestabilidad orbital (véase Ferrándiz y Martín [31]). Normalmente ambas técnicas se utilizan a la vez para, en primer lugar, transformar las ecuaciones en otras con soluciones estables, y en segundo lugar, integrar estas ecuaciones con un método numérico estable.

Dentro de estos métodos numéricos especiales podemos citar los métodos multirrevolución (véase Graf [51], Melendo y Palacios [87, 88] y Petzold [95]) y el método de Kirchgraber [67], diseñados para el cálculo a largo plazo de problemas orbitales. Otro grupo de métodos que podemos mencionar son los llamados P-estables. Podemos destacar entre los numerosos trabajos sobre este tipo de códigos los de Cash [10, 11], Chawla [13], Coleman [15], Coleman e Ixaru [16], Costabile y Costabile [17], Fatunla et al. [26], Franco y Palacios [38] e Ixaru y Paternoster [63]. 
Los integradores simplécticos han alcanzado gran popularidad en los últimos años. Estos métodos están construidos para la integración numérica de sistemas hamiltonianos y tienen la propiedad de conservar exactamente la 2-forma simpléctica de Poincaré-Cartan. Entre los últimos trabajos sobre estos métodos podemos destacar los de Chan y Murua [12], Hardy et al. [56], Li [71] y Sun [105]. Una buena referencia para estos integradores es el libro de Sanz-Serna y Calvo [97].

Van der Houwen y Sommeijer desarrollaron métodos multipaso $[59,60]$ minimizando el error de truncación para oscilaciones cuya frecuencia estuviese en un intervalo determinado y construyeron métodos Runge-Kutta [61, 62] con alto orden de dispersión para problemas oscilatorios.

Una técnica bastante habitual a la hora de desarrollar códigos numéricos especiales es cambiar el conjunto de funciones de referencia. Los códigos de propósito general utilizan los polinomios como referencia, mientras que las soluciones de un problema oscilatorio se aproximan más a otro tipo de funciones. En 1961 Gautschi [41] desarrolló una teoría tomando como funciones básicas las funciones trigonométricas. Brock y Murray [4] hicieron un estudio alternativo partiendo de las funciones exponenciales y Sheffield [101] desarrolló una teoría con distintos tipos de funciones básicas. En 1969 Stiefel y Bettis [102] modificaron el método de Cowell para integrar exactamente el problema de Kepler no perturbado eliminando así la inestabilidad de este método. Bettis [1,2] amplió su trabajo con Stiefel a los métodos de Störmer, Adams-Bashforth y Adams-Moulton de forma que los métodos modificados integrasen productos de polinomios ordinarios y polinomios de Fourier sin error de truncación. De Meyer et al. [18] desarrollaron una interpolación mixta a partir de la cual construyeron métodos numéricos de tipo multipaso (Van Daele et al. [107], Vanthournout et al. [111, 112]). Otros métodos que podemos citar que consiguen integrar exactamente funciones exponenciales son los de Ozawa [93], Paternoster [94], Van Daele et al. [108] y Vanden Berghe et al. [109, 110].

Mediante técnicas analíticas de las anteriormente citadas, las ecuaciones de un problema oscilatorio pueden ser reducidas a un sistema de osciladores perturbados. Por ejemplo, para el problema del satélite artificial, mediante las variables de Kustanheimo-Stiefel (véase Stiefel y Scheifele [103]) o mediante las variables de Burdet-Ferrándiz (véase 
Ferrándiz [28, 29] y Ferrándiz et al. [34]). Para problemas de este tipo Scheifele [98] obtuvo un refinamiento del método de Taylor basándose en un nuevo conjunto de funciones, las funciones $G$ (véase Martín et al. [84]). El error de truncación en el método de Scheifele contiene como factor el parámetro de perturbación y, por tanto, consigue integrar exactamente el problema no perturbado. A pesar de las buenas propiedades del método de Scheifele, su uso práctico está limitado a problemas con perturbaciones muy simples dada la complejidad de los cálculos previos que requiere (véase Fairén et al. [22]). Este problema fue solucionado por Martín y Ferrándiz [81, 82] transformando el método de Scheifele en un esquema multipaso (métodos SMF). Para problemas que no contengan explícitamente la derivada de la solución Martín y Farto [78] han obtenido una mejora de estos métodos, y López [72] han dado una reformulación de los métodos construidos por Jain et al. [65] (véase también Franco et al. [37]). A la vez se han obtenido extensiones de los métodos SMF a sistemas lineales de primer orden perturbados (Martín [77], y Martín y Ferrándiz [83]) y a problemas lineales perturbados de cualquier orden (López y Martín [73] y López et al. [75]). Un trabajo importante sobre este tipo de métodos es el de Vigo-Aguiar y Ferrándiz [114]. También, a partir de los métodos de Scheifele, se han obtenido métodos del tipo RungeKutta-Nyström (véase González [44] y González et al. [47, 49, 50]).

El buen comportamiento de gran parte de los algoritmos anteriormente citados depende del conocimiento previo de la frecuencia principal del problema oscilatorio o de una buena aproximación a ésta. Ferrándiz y Novo [33], basándose en el método de Lindstedt (véase Poincaré [96] o Verhulst [113]), introdujeron una técnica para la determinación precisa de la frecuencia que aplicaron a los métodos de Bettis consiguiendo que el error en la integración se mantuviese acotado en integraciones a largo plazo. Posteriormente esta técnica ha sido utilizada con éxito para la integración del problema del satélite artificial con diferentes esquemas numéricos (véase Ferrándiz et al. [32, 35] y Novo y Rojo [91, 92]). Recientemente Martín y Farto [79] han propuesto otra técnica para la determinación de la frecuencia basada en la primera aproximación de Kryloff-Bogoliuboff [68]. Utilizando esta técnica Martín y Velasco [86], y García et al. [40] han desarrollado procedimientos numéricos para obtener buenas aproximaciones a la frecuencia del problema. 
Un estudio comparativo de algunos de los métodos numéricos especiales anteriormente citados puede verse en Martín y Ferrándiz [80].

En esta memoria desarrollaremos nuevos esquemas numéricos mediante una pequeña modificación en los códigos clásicos de RungeKutta-Nyström. Introduciremos dicha modificación para mejorar el comportamiento de los métodos cuando integran problemas oscilatorios. La idea consiste en exigir a los nuevos métodos un orden más elevado cuando integran el problema del oscilador no perturbado sin llegar a exigir la integración exacta de éste, como lo hacen otros métodos especiales. La ventaja de los nuevos esquemas será la simplicidad de sus coeficientes, lo que les hacen muy recomendables para la integración con paso variable.

El primer capítulo de esta memoria es de carácter introductorio. En él presentamos los métodos clásicos de Runge-Kutta-Nyström y los métodos desarrollados por González et al. [47]. Realizamos este recordatorio ya que los métodos que se construyen en los capítulos siguientes puede considerarse que están en un punto intermedio entre ambos tipos de métodos.

El segundo capítulo está dedicado al desarrollo de unos nuevos métodos, que denominaremos por abreviar $\mathrm{RKN} h^{2}$, introduciendo el concepto de orden oscilatorio. Se ilustra la filosofía de dichos esquemas con la construccion y discusión de métodos de orden cuatro y órdenes oscilatorios cinco y seis, haciendo hincapié en las ventajas que presentan estos algoritmos frente a los esquemas Runge-KuttaNyström clásicos cuando se trata de integrar el problema del oscilador armónico, que con el mismo número de etapas sólo es capaz de alcanzar orden cuatro. Se obtienen expresiones para el error de truncación local y a partir de ellas se construye el método de orden oscilatorio cinco óptimo. A lo largo de todo el capítulo se presentan diversos experimentos numéricos que ponen de manifiesto el excelente comportamiento de los algoritmos desarrollados a la hora de integrar numéricamente problemas oscilatorios.

En el tercer capítulo obtenemos métodos de paso variable y orden bajo. Con los ejemplos presentados se pone de manifiesto la ventaja que presentan los nuevos códigos cuando se implementan en paso variable frente a los esquemas debidos a Gonzalez et al. [47], especialmente diseñados para integrar problemas oscilatorios. Estos poseen coeficientes que dependen del tamaño de paso de forma com- 
plicada y requieren un coste de tiempo de CPU elevado en una implementación en paso variable. Debido a la sencillez de los coeficientes de los códigos que se construyen en esta memoria el coste computacional en tiempo de CPU es mucho menor. Además, estos métodos presentan un comportamiento mucho mejor que los métodos Runge-KuttaNyström clásicos a pesar de que éstos tienen coeficientes constantes.

Parte de los resultados presentados en los capítulos dos y tres están ya publicados en García et al. [39].

En el último capítulo se construyen métodos de orden alto. Para ello se hace necesario introducir previamente la teoría de árboles especiales de Nyström con raíz debida a Hairer y Wanner [55] (véase también Butcher [6] y Hairer et al. $[54,55])$ y adaptarla a los métodos que nos ocupan en la presente memoria. De nuevo los experimentos numéricos realizados nos muestran unos resultados de los nuevos algoritmos altamente satisfactorios. 


\section{Capítulo 1}

\section{Métodos de un paso para la integración de ecuaciones de segundo orden}

\section{$1.1 \quad$ Introducción}

La mayor parte de los métodos numéricos existentes han sido diseñados para la integración de sistemas de ecuaciones diferenciales de primer orden. Sin embargo, en la práctica, muchos problemas dan lugar a ecuaciones de segundo orden. Los sistemas dinámicos están basados en fuerzas que provocan aceleraciones, que no son más que la derivada segunda de la posición. Una posibilidad, para la integración de ecuaciones de segundo orden, es la reducción de éstas a un sistema de ecuaciones de primer orden. Pero, debido a la gran cantidad de problemas que se modelan mediante ecuaciones de segundo orden, parece conveniente el desarrollo de métodos específicos para estas ecuaciones.

En este capítulo nos centraremos en métodos numéricos de un paso. En primer lugar presentaremos los métodos clásicos de Runge-KuttaNyström (RKN) y en una segunda parte los métodos desarrollados por González et al. [47] (RKGM) para la integración de osciladores perturbados. Presentamos estos dos tipos de algoritmos porque, de alguna forma y como se verá a lo largo de esta memoria, los nuevos métodos que vamos a introducir podría considerarse que se sitúan en un punto intermedio entre los métodos RKN y los RKGM. 


\subsection{Métodos Runge-Kutta-Nyström}

Consideremos el problema de valores iniciales

$$
y^{\prime \prime}=f\left(x, y, y^{\prime}\right), \quad y\left(x_{0}\right)=y_{0}, \quad y^{\prime}\left(x_{0}\right)=y_{0}^{\prime} .
$$

Si reescribimos la ecuación diferencial de segundo orden como un sistema de ecuaciones diferenciales de primer orden obtenemos

$$
\frac{d}{d x}\left(\begin{array}{c}
y \\
y^{\prime}
\end{array}\right)=\left(\begin{array}{c}
y^{\prime} \\
f\left(x, y, y^{\prime}\right)
\end{array}\right) .
$$

Aplicando una fórmula Runge-Kutta para sistemas de primer orden obtenemos la siguiente aproximación numérica:

$$
\begin{aligned}
& y_{1}=y_{0}+h \sum_{i=1}^{s} b_{i} \bar{k}_{i}, \\
& y_{1}^{\prime}=y_{0}^{\prime}+h \sum_{i=1}^{s} b_{i} k_{i}, \\
& \bar{k}_{i}=y_{0}^{\prime}+h \sum_{j=1}^{i-1} \bar{a}_{i j} k_{j}, \\
& k_{i}=f\left(x_{0}+c_{i} h, y_{0}+h \sum_{j=1}^{i-1} \bar{a}_{i j} \bar{k}_{j}, y_{0}^{\prime}+h \sum_{j=1}^{i-1} \bar{a}_{i j} k_{j}\right), \\
& \qquad i=1, \ldots, s .
\end{aligned}
$$

Insertando (1.4) en (1.2), (1.3) y (1.5), obtenemos la expresión del siguiente método

$$
\begin{aligned}
& y_{1}=y_{0}+h y_{0}^{\prime}+h^{2} \sum_{i=1}^{s} \bar{b}_{i} k_{i}, \\
& y_{1}^{\prime}=y_{0}^{\prime}+h \sum_{i=1}^{s} b_{i} k_{i}, \\
& k_{i}=f\left(x_{0}+c_{i} h, y_{0}+c_{i} h y_{0}^{\prime}+h^{2} \sum_{j=1}^{i-1} a_{i j} k_{j}, y_{0}^{\prime}+h \sum_{j=1}^{i-1} \bar{a}_{i j} k_{j}\right), \\
& i=1, \ldots, s,
\end{aligned}
$$

con

$$
a_{i j}=\sum_{k} \bar{a}_{i k} \bar{a}_{k j} \quad \mathrm{y} \quad \bar{b}_{i}=\sum_{j} b_{j} \bar{a}_{j i}
$$


Estos métodos de tipo Runge-Kutta diseñados para ecuaciones diferenciales de segundo orden se denominan habitualmente métodos RungeKutta-Nyström ya que fueron introducidos por Nyström en 1925, aunque Nyström construyó métodos que no satisfacían necesariamente las condiciones (1.6) (véase Dormand [19]). Un gran número de este tipo de métodos han sido desarrollados por Dormand et al. [20, 21], Fehlberg [27], Fine [36], Hairer [52, 53] y Sharp y Fine [99, 100]. Para este tipo de métodos se suelen usar las abreviaturas RKNG o RKN, aunque habitualmente se reserva esta última abreviatura para los métodos específicamente diseñados para problemas en los que la función no contiene explícitamente a la derivada de la solución (véase Dormand [19] y Hairer et al. [54]):

$$
y^{\prime \prime}=f(x, y), \quad y\left(x_{0}\right)=y_{0}, y^{\prime}\left(x_{0}\right)=y_{0}^{\prime} .
$$

Para este tipo de problemas las fórmulas del método serían de la siguiente forma

$$
\begin{aligned}
& y_{1}=y_{0}+h y_{0}^{\prime}+h^{2} \sum_{i=1}^{s} \bar{b}_{i} k_{i}, \\
& y_{1}^{\prime}=y_{0}^{\prime}+h \sum_{i=1}^{s} b_{i} k_{i}, \\
& k_{i}=f\left(x_{0}+c_{i} h, y_{0}+c_{i} h y_{0}^{\prime}+h^{2} \sum_{j=1}^{i-1} a_{i j} k_{j}\right), i=1, \ldots, s .
\end{aligned}
$$

Nótese que los coeficientes $\bar{a}_{i j}$ ya no aparecen en las fórmulas del algoritmo. En este caso, puede alcanzarse una gran simplificación tanto en la implementación del método como en el coste computacional requerido. El interés de estas fórmulas radica en el amplio espectro de problemas que pueden reducirse a ecuaciones de este tipo tras un adecuado cambio de variables.

A menudo los coeficientes de un método Runge-Kutta-Nyström se disponen en tablas que ayudan a visualizar el método como queda reflejado en la Tabla 1.1.

Para métodos de tipo RKN específicamente diseñados para la integración numérica de ecuaciones diferenciales de segundo orden se suele definir el orden del método de la siguiente forma. 


\begin{tabular}{cc|ccccc} 
& $c_{1}$ & & & $a_{i j}$ & & \\
$c_{i}$ & $c_{2}$ & $a_{21}$ & & & & \\
& $\vdots$ & $\vdots$ & $\ddots$ & & & \\
& $c_{s}$ & $a_{s 1}$ & $a_{s 2}$ & $\ldots$ & $a_{s s-1}$ & \\
\hline & $\bar{b}_{i}$ & $\bar{b}_{1}$ & $\bar{b}_{2}$ & $\ldots$ & $\bar{b}_{s-1}$ & $\bar{b}_{s}$ \\
\hline & $b_{i}$ & $b_{1}$ & $b_{2}$ & $\ldots$ & $b_{s-1}$ & $b_{s}$
\end{tabular}

Tabla 1.1: Método Runge-Kutta-Nyström.

Definición 1.2.1 Se dice que un método RKN tiene orden $p$ si para problemas suficientemente regulares se verifica

$$
\begin{gathered}
y\left(x_{0}+h\right)-y_{1}=O\left(h^{p+1}\right), \\
y^{\prime}\left(x_{0}+h\right)-y_{1}^{\prime}=O\left(h^{p+1}\right) .
\end{gathered}
$$

Llegados a este punto, una tarea importante pero compleja, es la derivación de condiciones de orden para métodos RKN. Con el fin de simplificar los cálculos se recurre a ciertas funciones y a una teoría de grafos principalmente resultado de los avances realizados por Butcher y posteriormente por Hairer y Wanner, que en este momento no trataremos, pero que adaptaremos más adelante a una nueva familia de esquemas de un paso. No obstante podemos remitirnos a Butcher [5, 6], Hairer et al. [54] y Hairer y Wanner [55], donde se proporciona una detallada y rigurosa exposición de las técnicas antes citadas para métodos Runge-Kutta y Runge-Kutta-Nyström y a Calvo y Sanz-Serna [9] donde se cuenta el número de condiciones de orden independientes tanto para métodos RKN como RKN simplécticos.

\subsection{Métodos RKGM}

La frecuencia con que se presentan en las ciencias aplicadas problemas que se modelizan mediante osciladores perturbados:

$$
y^{\prime \prime}+\omega^{2} y=\varepsilon g(x, y), \quad y\left(x_{0}\right)=y_{0}, \quad y^{\prime}\left(x_{0}\right)=y_{0}^{\prime},
$$


hace interesante la labor de desarrollar métodos numéricos específicos para los mismos. Así, el método de funciones $G$ de Scheifele [98] sirvió como punto de partida para desarrollar por parte de Martín y Ferrándiz [82] un tipo de métodos multipaso (SMF) que presentaba notables mejoras en la integración de osciladores perturbados con respecto a los desarrollados hasta entonces. Con la misma idea González et al. [44, 47, 50] desarrollaron fórmulas de tipo RK para estos mismos problemas (métodos RKGM). Vamos describir brevemente estos métodos.

Consideremos el problema de Cauchy (1.9). La solución de este problema se puede representar mediante una serie de funciones $G$ de Scheifele como sigue:

$$
y_{0} G_{0}\left(x-x_{0}\right)+y_{0}^{\prime} G_{1}\left(x-x_{0}\right)+\varepsilon \sum_{j=0}^{\infty} g_{0}^{(j)} G_{j+2}\left(x-x_{0}\right),
$$

donde las funciones $G$ de Scheifele se definen como las soluciones de los problemas de valores iniciales siguientes:

$$
\begin{aligned}
& G_{0}^{\prime \prime}+\omega^{2} G_{0}=0, \quad G_{0}(0)=1, \quad G_{0}^{\prime}(0)=0, \\
& G_{1}^{\prime \prime}+\omega^{2} G_{1}=0, \quad G_{1}(0)=0, \quad G_{1}^{\prime}(0)=1, \\
& G_{k}^{\prime \prime}+\omega^{2} G_{k}=\frac{x^{k-2}}{(k-2) !}, \quad G_{k}(0)=0, \quad G_{k}^{\prime}(0)=0, \quad k \geq 2 .
\end{aligned}
$$

y donde

$$
g_{0}^{(j)}=\left.\frac{d^{j}}{d x^{j}} g(x, y(x))\right|_{x=x_{0}} .
$$

Si esta expresión se trunca de forma que intervengan las $p-2$ primeras derivadas de la función $g$, podemos aproximar la solución y su derivada por:

$$
\begin{aligned}
& y_{1}=y_{0} G_{0}\left(x-x_{0}\right)+y_{0}^{\prime} G_{1}\left(x-x_{0}\right)+\varepsilon \sum_{k=2}^{p} g_{0}^{(k-2)} G_{k}\left(x-x_{0}\right), \\
& y_{1}^{\prime}=y_{0}^{\prime} G_{0}\left(x-x_{0}\right)-\omega^{2} y_{0} G_{1}\left(x-x_{0}\right)+\varepsilon \sum_{k=2}^{p} g_{0}^{(k-2)} G_{k-1}\left(x-x_{0}\right),
\end{aligned}
$$

que alcanza un orden menos en la aproximación a la derivada de la solución que en la correspondiente a la posición. Sustituyendo las 
derivadas de la función que intervienen en (1.10) y (1.11) por combinaciones lineales de evaluaciones de función en diversos puntos, podemos conseguir convertir el anterior esquema en un algoritmo que presenta grandes analogías con los métodos Runge-Kutta-Nyström. Para más detalles se puede consultar González [44], González et al. [45], López [72], Martín [77] y Scheifele [98].

Para fijar ideas, consideremos el método de Scheifele con $p=4$, en el que intervienen las dos primeras derivadas de la función, y consideremos los puntos $x_{0}, x_{0}+h / 2$ y $x_{0}+h$. Como candidatos a evaluaciones de función tomamos:

$$
\begin{aligned}
& k_{1} \approx g\left(x_{0}, y\left(x_{0}\right)\right), \\
& k_{2} \approx g\left(x_{0}+\frac{h}{2}, y\left(x_{0}+\frac{h}{2}\right)\right) \\
& k_{3} \approx g\left(x_{0}+h, y\left(x_{0}+h\right)\right) .
\end{aligned}
$$

El problema que se nos presenta ahora es aproximar la solución en los puntos que hemos seleccionado.

El primer valor es un dato del problema planteado y que denotaremos por

$$
y\left(x_{0}\right)=y_{0}=: \tilde{y}_{1}
$$

De esta forma

$$
k_{1}=g\left(x_{0}, y_{0}\right)=g_{0}
$$

Para el segundo valor podemos considerar

$$
\begin{aligned}
y\left(x_{0}+\frac{h}{2}\right) & =y_{0}+\frac{h}{2} y_{0}^{\prime}+\frac{h^{2}}{8}\left(\varepsilon g_{0}-\omega^{2} y_{0}\right)+O\left(h^{3}\right) \\
& =y_{0}+\frac{h}{2} y_{0}^{\prime}+\frac{h^{2}}{8}\left(\varepsilon k_{1}-\omega^{2} \tilde{y}_{1}\right)+O\left(h^{3}\right)
\end{aligned}
$$

y definiendo

$$
\tilde{y}_{2}:=y_{0}+\frac{h}{2} y_{0}^{\prime}+\frac{h^{2}}{8}\left(\varepsilon k_{1}-\omega^{2} \tilde{y}_{1}\right),
$$

consideramos

$$
k_{2}=g\left(x_{0}+\frac{h}{2}, \tilde{y}_{2}\right)
$$


Un razonamiento semejante al anterior nos permite aproximar la solución en $x_{0}+h$ de la siguiente forma:

$$
y\left(x_{0}+h\right) \approx y_{0}+h y_{0}^{\prime}+\frac{h^{2}}{2}\left(\varepsilon k_{2}-\omega^{2} \tilde{y}_{2}\right),
$$

definir

$$
\tilde{y}_{3}:=y_{0}+h y_{0}^{\prime}+\frac{h^{2}}{2}\left(\varepsilon k_{2}-\omega^{2} \tilde{y}_{2}\right),
$$

y tomar

$$
k_{3}=g\left(x_{0}+h, \tilde{y}_{3}\right) .
$$

Por tanto, las evaluaciones de función consideradas son

$$
\begin{aligned}
& k_{1}=g\left(x_{0}, y_{0}\right), \\
& \tilde{y}_{1}=y_{0}, \\
& k_{2}=g\left(x_{0}+\frac{1}{2} h, y_{0}+\frac{1}{2} h y_{0}^{\prime}+\frac{h^{2}}{8}\left(\varepsilon k_{1}-\omega^{2} \tilde{y}_{1}\right)\right), \\
& \tilde{y}_{2}=y_{0}+\frac{1}{2} h y_{0}^{\prime}+\frac{h^{2}}{8}\left(\varepsilon k_{1}-\omega^{2} \tilde{y}_{1}\right), \\
& k_{3}=g\left(x_{0}+h, y_{0}+h y_{0}^{\prime}+\frac{h^{2}}{2}\left(\varepsilon k_{2}-\omega^{2} \tilde{y}_{2}\right)\right) .
\end{aligned}
$$

Nuestro propósito ahora será aproximar las derivadas de la función $g$ de la siguiente forma:

$$
\begin{aligned}
g_{0}^{(0)}=g_{0} & \approx b_{01} k_{1}+b_{02} k_{2}+b_{03} k_{3}, \\
g_{0}^{(1)} & \approx b_{11} k_{1}+b_{12} k_{2}+b_{13} k_{3}, \\
& g_{0}^{(2)} \approx b_{21} k_{1}+b_{22} k_{2}+b_{23} k_{3} .
\end{aligned}
$$

Es claro que la mejor aproximación a $g_{0}$ se obtiene sin más que tomar $b_{01}=1$ y $b_{02}=b_{03}=0$, pues de esta forma se obtiene el valor exacto. Para determinar los valores $b_{j i}, j=1,2$, basta hacer un desarrollo de Taylor en torno a $h=0$ de los segundos miembros en (1.13) y (1.14) e identificar términos semejantes con $g_{0}^{(1)}$ y $g_{0}^{(2)}$ respectivamente. Ilustrémoslo con el cálculo de los coeficientes $b_{1 i}, \quad i=1,2,3$. 
Desarrollando en serie de Taylor el segundo miembro de (1.13) resulta

$$
\begin{aligned}
& b_{11} k_{1}+b_{12} k_{2}+b_{13} k_{3}=\sum_{i=1}^{3} b_{1 i} g_{0}+\frac{h}{2} \sum_{i=2}^{3} b_{1 i}\left(g_{x}+g_{y} y_{0}^{\prime}\right) \\
& +\frac{h^{2}}{2} \sum_{i=2}^{3} b_{1 i}\left(g_{x x}+2 g_{x y} y_{0}^{\prime}+g_{y y} y_{0}^{\prime 2}+g_{y} g-\omega^{2} y_{0} g_{y}\right)+ \\
& O\left(h^{3}\right)
\end{aligned}
$$

donde por $g_{x}$ y $g_{y}$ denotamos las derivadas parciales de la función $g$ evaluadas en $x=x_{0}$.

Por otro lado, se verifica

$$
g_{0}^{(1)}=g_{x}+g_{y} y^{\prime}
$$

e identificando términos semejantes entre (1.15) y (1.16) obtenemos el siguiente sistema de tres ecuaciones:

$$
\left.\begin{array}{rcc}
b_{11}+b_{12}+b_{13} & = & 0 \\
b_{12}+2 b_{13} & = & 2 / h \\
b_{12}+4 b_{13} & = & 0
\end{array}\right\}
$$

Con solución $b_{11}=-3 / h, b_{12}=4 / h, b_{13}=-1 / h$. Razonando de la misma forma para obtener $b_{2 i}, \quad i=1,2,3$, resulta $b_{21}=4 / h^{2}, b_{22}=$ $-8 / h^{2}, b_{23}=4 / h^{2}$. Unos simples cálculos muestran que con estos valores, conseguimos aproximar las dos primeras derivadas de la función hasta orden 2 y que no es posible aproximar con dicho orden la derivada de orden tres de la función.

Sustituyendo en (1.10) y (1.11) las aproximaciones obtenidas, llegamos al siguiente método numérico:

$$
\begin{aligned}
y_{1}= & y_{0} G_{0}+y_{0}^{\prime} G_{1}+\varepsilon\left(k_{1} G_{2}+\left(-3 k_{1}+4 k_{2}-k_{3}\right) \frac{G_{3}}{h}+\right. \\
& \left.\left(4 k_{1}-8 k_{2}+4 k_{3}\right) \frac{G_{4}}{h^{2}}\right), \\
y_{1}^{\prime}= & y_{0}^{\prime} G_{0}-\omega^{2} y_{0} G_{1}+\varepsilon\left(k_{1} G_{1}+\left(-3 k_{1}+4 k_{2}-k_{3}\right) \frac{G_{2}}{h}+\right. \\
& \left.\left(4 k_{1}-8 k_{2}+4 k_{3}\right) \frac{G_{3}}{h^{2}}\right),
\end{aligned}
$$


que podemos reescribir como:

$$
\begin{aligned}
y_{1}= & y_{0} G_{0}+y_{0}^{\prime} G_{1}+\varepsilon\left(\left(G_{2}-3 \frac{G_{3}}{h}+4 \frac{G_{4}}{h^{2}}\right) k_{1}+\left(4 \frac{G_{3}}{h}-8 \frac{G_{4}}{h^{2}}\right) k_{2}\right. \\
& \left.+\left(-\frac{G_{3}}{h}+4 \frac{G_{4}}{h^{2}}\right) k_{3}\right), \\
y_{1}^{\prime}= & y_{0}^{\prime} G_{0}-\omega^{2} y_{0} G_{1}+\varepsilon\left(\left(G_{1}-3 \frac{G_{2}}{h}+4 \frac{G_{3}}{h^{2}}\right) k_{1}+\left(4 \frac{G_{2}}{h}-8 \frac{G_{3}}{h^{2}}\right) k_{2}\right. \\
& \left.+\left(-\frac{G_{2}}{h}+4 \frac{G_{3}}{h^{2}}\right) k_{3}\right) .
\end{aligned}
$$

De esta forma podemos visualizar el método obtenido como una versión de tipo Runge-Kutta del método de Scheifele, donde tras truncar la serie de funciones $G$, hemos sustituido las derivadas de la función $g$ por combinaciones de evaluaciones de función en los puntos $x_{0}, x_{0}+h / 2$ y $x_{0}+h$ con coeficientes

$$
b_{1}=G_{2}-3 \frac{G_{3}}{h}+4 \frac{G_{4}}{h^{2}}, b_{2}=4 \frac{G_{3}}{h}-8 \frac{G_{4}}{h^{2}}, b_{3}=-\frac{G_{3}}{h}+4 \frac{G_{4}}{h^{2}},
$$

en el caso de la aproximación a la posición y

$$
\bar{b}_{1}=G_{1}-3 \frac{G_{2}}{h}+4 \frac{G_{3}}{h^{2}}, \bar{b}_{2}=4 \frac{G_{2}}{h}-8 \frac{G_{3}}{h^{2}}, \bar{b}_{3}=-\frac{G_{2}}{h}+4 \frac{G_{3}}{h^{2}},
$$

en el caso de la aproximación a la velocidad.

Los métodos construidos de esta forma fueron denominados por sus autores RKGM (Runge-Kutta-G-functions-Methods). La definición formal de tales métodos es la siguiente

Definición 1.3.1 Sean $s \in \mathbb{Z}^{+}, p \in \mathbb{N}, a_{i j}, \quad \bar{a}_{i j} \quad i=1, \ldots, s, j=$ $1, \ldots, i-1, b_{j i}, j=1, \ldots, p-2, b_{01}=1, b_{0 i}=0, i \neq 1, c_{1}=0$ y $c_{i}$, $i=2 \ldots s$ coeficientes reales. Llamaremos método RKGM explícito de $s$ etapas y $p+1$ funciones $G$ para el problema

$$
y^{\prime \prime}+\omega^{2} y=\varepsilon g\left(x, y, y^{\prime}\right), \quad y\left(x_{0}\right)=y_{0}, y^{\prime}\left(x_{0}\right)=y_{0},
$$

al siguiente esquema

$$
\tilde{y}_{0}=y_{0}, \quad \tilde{y}_{i}=y_{0}+c_{i} h y_{0}^{\prime}+h^{2} \sum_{j=1}^{i-1} \bar{a}_{i j}\left(\varepsilon k_{j}-\omega^{2} \tilde{y}_{j}\right),
$$




$$
\begin{aligned}
& \tilde{y}_{0}^{\prime}=y_{0}^{\prime}, \quad \tilde{y}_{i}^{\prime}=y_{0}^{\prime}+h \sum_{j=1}^{i-1} a_{i j}\left(\varepsilon k_{j}-\omega^{2} \tilde{y}_{j}\right), \\
& k_{i}=g\left(x_{0}+c_{i} h, \tilde{y}_{i}, \tilde{y}_{i}^{\prime}\right), \quad i=1, \ldots, s, \\
& y_{1}=y_{0} G_{0}+y_{0}^{\prime} G_{1}+\varepsilon \sum_{i=1}^{s} \sum_{j=0}^{p-2} b_{j i} \frac{G_{j+2}}{h^{j}} k_{i}, \\
& y_{1}^{\prime}=y_{0}^{\prime} G_{0}-\omega^{2} y_{0} G_{1}+\varepsilon \sum_{i=1}^{s} \sum_{j=0}^{p-2} b_{j i} \frac{G_{j+1}}{h^{j}} k_{i} .
\end{aligned}
$$

Es interesante destacar que los métodos RKGM no suponen un incremento del coste computacional sobre los esquemas de tipo RKN cuando integramos con paso fijo, ya que la única diferencia con tal código se produce al comienzo de la integración numérica, momento en el que se calculan los coeficientes, que dependen en este caso del tamaño de paso $h$. Cuando se implementan en paso variable sí que aparecen diferencias ya que en cada cambio de paso hay que recalcular los coeficientes.

Otra propiedad interesante de tales métodos es que el error de truncación local aparece multiplicado por el parámetro de perturbación $\varepsilon$ (véase González et al. [47]). Por ello son muy efectivos cuando se trata de integrar osciladores débilmente perturbados ( $\varepsilon$ pequeño). 


\section{Capítulo 2}

\section{Nuevos métodos tipo Runge-Kutta-Nyström.}

\section{$2.1 \quad$ Introducción}

Nuestro interés se centra en la integración de problemas de valores iniciales cuyas ecuaciones sean osciladores perturbados de la forma:

$$
y^{\prime \prime}+\omega^{2} y=\varepsilon g(x, y), \quad y\left(x_{0}\right)=y_{0}, \quad y^{\prime}\left(x_{0}\right)=y_{0}^{\prime},
$$

siendo $\varepsilon$ un pequeño parámetro de perturbación. Como mencionamos anteriormente, muchos problemas de las ciencias aplicadas y de la técnica pueden ser formulados mediante sistemas de tales ecuaciones. Tomando $f(x, y)=\varepsilon g(x, y)-\omega^{2} y$ podemos escribir (2.1) como

$$
y^{\prime \prime}=f(x, y), \quad y\left(x_{0}\right)=y_{0}, \quad y^{\prime}\left(x_{0}\right)=y_{0}^{\prime},
$$

e integrar el problema con un método de propósito general como el esquema RKN (1.7) que tratamos en el capítulo anterior. Sin embargo resulta más conveniente la integración con alguno de los métodos especialmente diseñados para este tipo de ecuaciones. Entre estos métodos podemos mencionar los debidos a Bettis [1, 3], Franco et al. [37], González et al . [47], Jain et al. [65], López et al . [74], Martín y Ferrándiz [82], Paternoster [94] o Scheifele [98]. Todos ellos proporcionan excelentes resultados integrando el problema que nos ocupa. Mejores, en muchos casos, que los métodos de propósito general como el mencionado RKN. Sin embargo, presentan el inconveniente de que 
sus coeficientes no son tan sencillos como los de un RKN clásico. Entre los citados anteriormente, por ejemplo, los métodos de Bettis utilizan una relación de recurrencia para obtener dos de ellos y otros códigos requieren evaluar las funciones $G$ de Scheifele. Este inconveniente se hace verdaderamente serio si realizamos la integración en paso variable, pues entonces recalcular los coeficientes, que dependen del tamaño de paso de una forma complicada, lleva un importante coste computacional, que sólo es despreciable en problemas con funciones costosas de evaluar.

Así, si buscáramos una aproximación razonablemente buena a la solución de (2.2) estaríamos en la disyuntiva de usar un método clásico con un número de etapas, en general, elevado para obtener suficiente precisión, o por el contrario, utilizar los métodos especiales, que pueden resultar mucho más adecuados, pero cuyos coeficientes son más complicados y conllevan un incremento en coste computacional cuando se implementan en paso variable.

El objetivo de este trabajo es diseñar, a partir de un esquema clásico como el considerado en (1.7), nuevos métodos que integren eficientemente el problema que nos ocupa, pero con unos coeficientes mucho más sencillos. Estos coeficientes apenas incrementarán el coste computacional cuando se construya una rutina de paso variable. Para ello, partiendo de un método Runge-Kutta-Nyström introduciremos ciertas modificaciones en los coeficientes de modo que cuando la función $f$ del problema (2.2) sea una función cualquiera, el método sea capaz de alcanzar el mismo orden que el método Runge-Kutta-Nyström y cuando $f=-\omega^{2} y$ el método alcance mayor orden. De esta forma tendremos métodos que integren mejor que el código RKN original el oscilador no perturbado y, por tanto, cabe esperar tengan un buen comportamiento para el problema (2.1) cuando el parámetro de perturbación sea pequeño. A lo largo de la memoria nos será de gran utilidad la siguiente definición.

Definición 2.1.1 Diremos que un método numérico para integrar ecuaciones diferenciales de segundo orden tiene orden oscilatorio $q$ si posee dicho orden cuando integra el siguiente el problema de valores iniciales

$$
y^{\prime \prime}=-\omega^{2} y, \quad y\left(x_{0}\right)=y_{0}, \quad y^{\prime}\left(x_{0}\right)=y_{0}^{\prime}
$$


En definitiva, lo que perseguimos con los nuevos métodos es alcanzar el mismo orden que los esquemas RKN con igual número de etapas consiguiendo además, un orden oscilatorio mayor.

Nota 2.1.2 En lo sucesivo consideraremos las funciones $f$ y $g$ suficientemente regulares

\subsection{Nuevos métodos especiales para problemas oscilatorios}

Vamos a considerar un método del tipo (1.7) de 3 etapas para integrar el problema de Cauchy (2.2)

$$
\begin{aligned}
& k_{1}=f\left(x_{0}, y_{0}\right), \\
& k_{2}=f\left(x_{0}+h c_{2}, y_{0}+h c_{2} y_{0}^{\prime}+h^{2} a_{21} k_{1}\right), \\
& k_{3}=f\left(x_{0}+h c_{3}, y_{0}+h c_{3} y_{0}^{\prime}+h^{2}\left(a_{31} k_{1}+a_{32} k_{2}\right)\right), \\
& y_{1}=y_{0}+h y_{0}^{\prime}+h^{2}\left(\bar{b}_{1} k_{1}+\bar{b}_{2} k_{2}+\bar{b}_{3} k_{3}\right), \\
& y_{1}^{\prime}=y_{0}^{\prime}+h\left(b_{1} k_{1}+b_{2} k_{2}+b_{3} k_{3}\right) .
\end{aligned}
$$

Si exigimos que dicho algoritmo tenga orden cuatro tanto en la aproximación a la solución como a su derivada, de acuerdo con la Definición 1.2.1, es necesario que los errores locales cumplan las siguientes condiciones:

$$
\begin{aligned}
& y\left(x_{0}+h\right)-y_{1}=O\left(h^{5}\right), \\
& y^{\prime}\left(x_{0}+h\right)-y_{1}^{\prime}=O\left(h^{5}\right),
\end{aligned}
$$

siendo $y_{1}$ e $y_{1}^{\prime}$ las aproximaciones en $x=x_{0}+h$ a $y(x)$ e $y^{\prime}(x)$ respectivamente. Es decir, es necesario que los desarrollos de Taylor de la solución exacta y la numérica coincidan hasta el término en $h^{4}$, y del mismo modo, en la derivada de la solución.

Podemos expresar la solución exacta y su derivada según su desarrollo de Taylor

$$
\begin{gathered}
y\left(x_{0}+h\right)=y\left(x_{0}\right)+h y^{\prime}\left(x_{0}\right)+\frac{h^{2}}{2 !} y^{\prime \prime}\left(x_{0}\right)+\frac{h^{3}}{3 !} y^{\prime \prime \prime}\left(x_{0}\right)+O\left(h^{4}\right), \\
y^{\prime}\left(x_{0}+h\right)=y^{\prime}\left(x_{0}\right)+h y^{\prime \prime}\left(x_{0}\right)+\frac{h^{2}}{2 !} y^{\prime \prime \prime}\left(x_{0}\right)+\frac{h^{3}}{3 !} y^{(i v)}\left(x_{0}\right)+O\left(h^{4}\right),
\end{gathered}
$$


y reemplazando $y^{\prime \prime}$ por $f$ cada vez que aparece de acuerdo con (2.2), resulta

$$
\begin{aligned}
y\left(x_{0}+h\right) & =y_{0}+h y_{0}^{\prime}+\frac{h^{2}}{2 !} f\left(x_{0}, y_{0}\right)+\frac{h^{3}}{3 !}\left(f_{x}+f_{y} y^{\prime}\right)\left(x_{0}, y_{0}\right)+ \\
& +\frac{h^{4}}{4 !}\left(f_{x x}+2 f_{x y} y^{\prime}+f_{y y}\left(y^{\prime}\right)^{2}+f_{y} f\right)\left(x_{0}, y_{0}\right)+O\left(h^{5}\right), \\
y^{\prime}\left(x_{0}+h\right) & =y_{0}^{\prime}+h f\left(x_{0}, y_{0}\right)+\frac{h^{2}}{2 !}\left(f_{x}+f_{y} y^{\prime}\right)\left(x_{0}, y_{0}\right)+ \\
& +\frac{h^{3}}{3 !}\left(f_{x x}+2 f_{x y} y^{\prime}+f_{y y}\left(y^{\prime}\right)^{2}+f_{y} f\right)\left(x_{0}, y_{0}\right) \\
& +\frac{h^{4}}{4 !}\left(f_{x x x}+3 f_{x x y} y^{\prime}+3 f_{x y y}\left(y^{\prime}\right)^{2}+f_{y y y}\left(y^{\prime}\right)^{3}\right. \\
& \left.+3 f_{y y} f y^{\prime}+3 f_{x y} f+f_{x} f_{y}+f_{y}^{2} y^{\prime}\right)\left(x_{0}, y_{0}\right)+O\left(h^{5}\right),
\end{aligned}
$$

Desarrollando en torno a $h=0$ y restando obtenemos para los errores las siguientes expresiones:

$$
\begin{aligned}
y\left(x_{0}+h\right)-y_{1} & =h^{2}\left(\frac{1}{2}-\bar{b}_{1}-\bar{b}_{2}-\bar{b}_{3}\right) F_{1}^{(2)}\left(x_{0}, y_{0}\right) \\
& +h^{3}\left(\frac{1}{6}-\bar{b}_{2} c_{2}-\bar{b}_{3} c_{3}\right) F_{1}^{(3)}\left(x_{0}, y_{0}, y_{0}^{\prime}\right) \\
& +h^{4}\left[\left(\frac{1}{24}-\frac{1}{2} \bar{b}_{2} c_{2}^{2}-\frac{1}{2} \bar{b}_{3} c_{3}^{2}\right) F_{1}^{(4)}\left(x_{0}, y_{0}, y_{0}^{\prime}\right)\right. \\
& \left.+\left(\frac{1}{24}-\bar{b}_{2} a_{21}-\bar{b}_{3}\left(a_{31}+a_{32}\right)\right) F_{2}^{(4)}\left(x_{0}, y_{0}, y_{0}^{\prime}\right)\right] \\
& +O\left(h^{5}\right), \\
y^{\prime}\left(x_{0}+h\right)-y_{1} & =h\left(1-b_{1}-b_{2}-b_{3}\right) F_{1}^{(2)}\left(x_{0}, y_{0}, y_{0}^{\prime}\right) \\
& +h^{2}\left(\frac{1}{2}-b_{2} c_{2}-b_{3} c_{3}\right) F_{1}^{(3)}\left(x_{0}, y_{0}, y_{0}^{\prime}\right) \\
& +h^{3}\left[\left(\frac{1}{6}-\frac{1}{2} b_{2} c_{2}^{2}-\frac{1}{2} b_{3} c_{3}^{2}\right) F_{1}^{(4)}\left(x_{0}, y_{0}, y_{0}^{\prime}\right)\right. \\
& \left.+\left(\frac{1}{6}-b_{2} a_{21}-b_{3}\left(a_{31}+a_{32}\right)\right) F_{2}^{(4)}\left(x_{0}, y_{0}, y_{0}^{\prime}\right)\right] \\
& +h^{4}\left[\left(\frac{1}{24}-\frac{1}{6} b_{2} c_{2}^{3}-\frac{1}{6} b_{3} c_{3}^{3}\right) F_{1}^{(5)}\left(x_{0}, y_{0}, y_{0}^{\prime}\right)\right. \\
& +\left(\frac{1}{8}-b_{2} c_{2} a_{21}-b_{3} c_{3}\left(a_{21}+a_{31}\right)\right) F_{2}^{(5)}\left(x_{0}, y_{0}, y_{0}^{\prime}\right) \\
& \left.\left.+\left(\frac{1}{24}-b_{3} c_{2} a_{32}\right) F_{3}^{(5)}\left(x_{0}, y_{0}, y_{0}^{\prime}\right)\right)\right]+O\left(h^{5}\right) .
\end{aligned}
$$


Para simplificar la notación hemos introducido las diferenciales elementales para ecuaciones de segundo orden, que se obtienen de forma similar a las de un proceso Runge-Kutta para ecuaciones de primer orden derivando $y^{\prime \prime}=f(x, y(x))$ sucesivamente con respecto a $x$ (véase Butcher [5] y Dormand [19])

$$
\begin{aligned}
F_{1}^{(2)} & =f \\
F_{1}^{(3)} & =f_{x}+f_{y} y^{\prime} \\
F_{1}^{(4)} & =f_{x x}+2 f_{x y} y^{\prime}+f_{y y}\left(y^{\prime}\right)^{2} \\
F_{2}^{(4)} & =f_{y} f \\
F_{1}^{(5)} & =f_{x x x}+3 f_{x x y} y^{\prime}+3 f_{x y y}\left(y^{\prime}\right)^{2}+f_{y y y}\left(y^{\prime}\right)^{3} \\
F_{2}^{(5)} & =f_{y y} f y^{\prime}+f_{x y} f \\
F_{3}^{(5)} & =f_{x} f_{y}+f_{y}^{2} y^{\prime} \\
F_{1}^{(6)} & =f_{x x x x}+4 f_{x x x y} y^{\prime}+6 f_{x x y y}\left(y^{\prime}\right)^{2}+4 f_{x y y y}\left(y^{\prime}\right)^{3}+f_{y y y y}\left(y^{\prime}\right)^{4}, \\
F_{2}^{(6)} & =f_{x x y} f+2 f_{x y y} f y^{\prime}+f_{y y y} f\left(y^{\prime}\right)^{2} \\
F_{3}^{(6)} & =f_{y y} f^{2} \\
F_{4}^{(6)} & =f_{y y} f_{y}\left(y^{\prime}\right)^{2}+f_{y} f_{x y} y^{\prime}+f_{x y} f_{x}+f_{y y} f_{x} y^{\prime}, \\
F_{5}^{(6)} & =f_{y y}\left(y^{\prime}\right)^{2}+2 f_{y} f_{x y} y^{\prime}+f_{x x} f_{y}, \\
F_{6}^{(6)} & =f_{y}^{2} f \\
& \cdots
\end{aligned}
$$

Para alcanzar orden cuatro tenemos que anular los coeficientes hasta $h^{4}$ inclusive. Esto nos lleva a las siguientes ecuaciones de orden

$$
\begin{aligned}
\bar{b}_{1}+\bar{b}_{2}+\bar{b}_{3} & =1 / 2, \\
\bar{b}_{2} c_{2}+\bar{b}_{3} c_{3} & =1 / 6, \\
\bar{b}_{2} c_{2}^{2}+\bar{b}_{3} c_{3}^{2} & =1 / 12, \\
\bar{b}_{2} a_{21}+\bar{b}_{3}\left(a_{31}+a_{32}\right) & =1 / 24, \\
b_{1}+b_{2}+b_{3} & =1, \\
b_{2} c_{2}+b_{3} c_{3} & =1 / 2, \\
b_{2} c_{2}^{2}+b_{3} c_{3}^{2} & =1 / 3, \\
b_{2} a_{21}+b_{3}\left(a_{31}+a_{32}\right) & =1 / 6, \\
b_{2} c_{2}^{3}+b_{3} c_{3}^{3} & =1 / 4,
\end{aligned}
$$




$$
\begin{aligned}
b_{2} c_{2} a_{21}+b_{3} c_{3}\left(a_{31}+a_{32}\right) & =1 / 8, \\
b_{3} c_{2} a_{32} & =1 / 24 .
\end{aligned}
$$

Podemos utilizar las clásicas condiciones de suma de fila

$$
\begin{aligned}
a_{21} & =\frac{1}{2} c_{2}^{2}, \\
a_{31}+a_{32} & =\frac{1}{2} c_{3}^{2},
\end{aligned}
$$

y otras condiciones simplificadoras que aparecen frecuentemente en la bibliografía (véase Dormand [19], Dormand et al. [20, 21], Hairer $[52,53]$ y Hairer et al. [54])

$$
\begin{array}{r}
\bar{b}_{1}=b_{1}, \\
\bar{b}_{2}=b_{2}\left(1-c_{2}\right), \\
\bar{b}_{3}=b_{3}\left(1-c_{3}\right) .
\end{array}
$$

De esta forma se reduce el número de ecuaciones de orden que hay que resolver. Esto es bastante habitual cuando el orden del método es alto y el número de ecuaciones es tan grande que se hacen necesarias estas condiciones simplificadoras para que el sistema resulte más manejable. Resolviendo el sistema anterior se obtendría una familia uniparamétrica de métodos. Podríamos tratar de utilizar el parámetro libre para satisfacer las ecuaciones adicionales que surgen al imponer orden 5. Sin embargo, el número de coeficientes es entonces inferior al número de ecuaciones y el sistema resultante es incompatible.

Vamos a considerar ahora el problema del oscilador no perturbado tomando como función $f(x, y)=-\omega^{2} y$ en (2.2). Para este caso particular las diferenciales elementales se simplifican mucho, quedando los términos en los que aparece sólo $f_{y}=-\omega^{2}$ o cualquiera de sus potencias. Así

$$
\begin{aligned}
y^{\prime \prime} & =-\omega^{2} y, \\
y^{\prime \prime \prime} & =-\omega^{2} y^{\prime}, \\
y^{(i v)} & =\omega^{4} y, \\
y^{(v)} & =\omega^{4} y^{\prime}, \\
y^{(v i)} & =-\omega^{6} y, \\
y^{(v i i)} & =-\omega^{6} y^{\prime}, \\
& \ldots
\end{aligned}
$$


Tomando en las correspondientes series de Taylor los términos en $h^{5}$ y $h^{6}$ y sustituyendo (2.22) obtenemos para los métodos (2.4)

$$
\begin{array}{r}
\omega^{4} y_{0}^{\prime}\left(\bar{b}_{3} a_{32} c_{2}-1 / 120\right) h^{5}, \\
-\omega^{6} y_{0}\left(\bar{b}_{3} a_{32} a_{21}-1 / 720\right) h^{6}, \\
-\omega^{6} y_{0}\left(b_{3} a_{32} a_{21}-1 / 120\right) h^{5}, \\
\frac{\omega^{6} y_{0}^{\prime}}{720} h^{6} .
\end{array}
$$

Las expresiones (2.23) y (2.24) corresponden al error en la aproximación a solución y (2.25) y (2.26) al error en la aproximación a la derivada. El término (2.26) no se anula en general, por lo que sería imposible alcanzar orden oscilatorio 6 . Por otra parte, para obtener orden oscilatorio 5 con el método (2.4), sería necesario resolver el sistema de ecuaciones

$$
\begin{aligned}
\bar{b}_{3} a_{32} c_{2} & =1 / 120, \\
b_{3} a_{32} a_{21} & =1 / 120 .
\end{aligned}
$$

Si sustituimos los coeficientes del método en función del parámetro libre, obtendríamos de nuevo un sistema sin solución. Por tanto, con el esquema RKN que estamos considerando no se puede alcanzar orden oscilatorio 5 .

Consideremos ahora un nuevo método que se obtiene a partir del esquema RKN (2.4), conservando las mismas etapas y añadiendo unos nuevos coeficientes $\bar{b}_{i}^{*}$ y $b_{i}^{*}$ multiplicados por $h^{2}$ :

$$
\begin{aligned}
& k_{1}=f\left(x_{0}, y_{0}\right), \\
& k_{2}=f\left(x_{0}+h c_{2}, y_{0}+h c_{2} y_{0}^{\prime}+h^{2} a_{21} k_{1}\right), \\
& k_{3}=f\left(x_{0}+h c_{3}, y_{0}+h c_{3} y_{0}^{\prime}+h^{2}\left(a_{31} k_{1}+a_{32} k_{2}\right)\right), \\
& y_{1}=y_{0}+h y_{0}^{\prime}+h^{2}\left(\left(\bar{b}_{1}+h^{2} \bar{b}_{1}^{*}\right) k_{1}+\left(\bar{b}_{2}+h^{2} \bar{b}_{2}^{*}\right) k_{2}+\left(\bar{b}_{3}+h^{2} \bar{b}_{3}^{*}\right) k_{3}\right), \\
& y_{1}^{\prime}=y_{0}^{\prime}+h\left(\left(b_{1}+h^{2} b_{1}^{*}\right) k_{1}+\left(b_{2}+h^{2} b_{2}^{*}\right) k_{2}+\left(b_{3}+h^{2} b_{3}^{*}\right) k_{3}\right) .
\end{aligned}
$$

Desarrollando de nuevo en serie de Taylor tenemos

$$
\begin{aligned}
y\left(x_{0}+h\right)-y_{1} & =h^{2}\left(\frac{1}{2}-\bar{b}_{1}-\bar{b}_{2}-\bar{b}_{3}\right) F_{1}^{(2)}\left(x_{0}, y_{0}, y_{0}^{\prime}\right) \\
& +h^{3}\left(\frac{1}{6}-\bar{b}_{2} c_{2}-\bar{b}_{3} c_{3}\right) F_{1}^{(3)}\left(x_{0}, y_{0}, y_{0}^{\prime}\right)
\end{aligned}
$$




$$
\begin{aligned}
& +h^{4}\left[\left(\frac{1}{24}-\frac{1}{2} \bar{b}_{2} c_{2}^{2}-\frac{1}{2} \bar{b}_{3} c_{3}^{2}\right) F_{1}^{(4)}\left(x_{0}, y_{0}, y_{0}^{\prime}\right)\right. \\
& +\left(\frac{1}{24}-\bar{b}_{2} a_{21}-\bar{b}_{3}\left(a_{31}+a_{32}\right)\right) F_{2}^{(4)}\left(x_{0}, y_{0}, y_{0}^{\prime}\right) \\
& \left.+\left(\bar{b}_{1}^{*}+\bar{b}_{2}^{*}+\bar{b}_{3}^{*}\right) F_{1}^{(2)}\left(x_{0}, y_{0}, y_{0}^{\prime}\right)\right]+O\left(h^{5}\right), \\
y^{\prime}\left(x_{0}+h\right)-y_{1} & =h\left(1-b_{1}-b_{2}-b_{3}\right) F_{1}^{(2)}\left(x_{0}, y_{0}, y_{0}^{\prime}\right) \\
& +h^{2}\left(\left(\frac{1}{2}-b_{2} c_{2}-b_{3} c_{3}\right) F_{1}^{(3)}\left(x_{0}, y_{0}, y_{0}^{\prime}\right)\right. \\
& +h^{3}\left[\left(\frac{1}{6}-\frac{1}{2} b_{2} c_{2}^{2}-\frac{1}{2} b_{3} c_{3}^{2}\right) F_{1}^{(4)}\left(x_{0}, y_{0}, y_{0}^{\prime}\right)\right. \\
& +\left(\frac{1}{6}-b_{2} a_{21}-b_{3}\left(a_{31}+a_{32}\right)\right) F_{2}^{(4)}\left(x_{0}, y_{0}, y_{0}^{\prime}\right) \\
& \left.+\left(b_{1}^{*}+b_{2}^{*}+b_{3}^{*}\right) F_{1}^{(2)}\left(x_{0}, y_{0}, y_{0}^{\prime}\right)\right] \\
& +h^{4}\left[\left(\frac{1}{24}-\frac{1}{6} b_{2} c_{2}^{3}-\frac{1}{6} b_{3} c_{3}^{3}\right) F_{1}^{(5)}\left(x_{0}, y_{0}, y_{0}^{\prime}\right)\right. \\
& +\left(\frac{1}{8}-b_{2} c_{2} a_{21}-b_{3} c_{3}\left(a_{21}+a_{31}\right)\right) F_{2}^{(5)}\left(x_{0}, y_{0}, y_{0}^{\prime}\right) \\
& \left.+\left(\frac{1}{24}-b_{3} c_{2} a_{32}\right) F_{3}^{(5)}\left(x_{0}, y_{0}, y_{0}^{\prime}\right)\right) \\
& \left.+\left(b_{2}^{*} c_{2}+b_{3}^{*} c_{3}\right) F_{1}^{(3)}\left(x_{0}, y_{0}, y_{0}^{\prime}\right)\right]+O\left(h^{5}\right),
\end{aligned}
$$

e imponiendo orden cuatro tendríamos las ecuaciones (2.6)-(2.16) junto con otras tres, que surgen como resultado de la nueva estructura del método

$$
\begin{aligned}
\bar{b}_{1}^{*}+\bar{b}_{2}^{*}+\bar{b}_{3}^{*} & =0, \\
b_{1}^{*}+b_{2}^{*}+b_{3}^{*} & =0, \\
b_{2}^{*} c_{2}+b_{3}^{*} c_{3} & =0 .
\end{aligned}
$$

Resolviendo el sistema obtendremos métodos de orden cuatro para el problema (2.2).

Llegados a este punto resaltamos dos importantes ventajas que presenta este método frente al clásico:

1. Debido a que los nuevos métodos cuentan con más parámetros que el esquema clásico esto nos permitirá, con el mismo número de etapas, alcanzar orden oscilatorio 5. 
2. Por otro lado, los nuevos coeficientes $\bar{b}_{i}^{*}$ y $b_{i}^{*}$ van multiplicados por $h^{2}$ entonces, aparecen las mismas relaciones que en los correspondientes $\bar{b}_{i}$ y $b_{i}$ pero multiplicando a $F_{j}^{(k+2)}$ en lugar de a $F_{j}^{(k)}$. Para el problema que nos interesa, teniendo en cuenta (2.22) es sencillo ver que $y^{(k+2)}+\omega^{2} y^{(k)}=0$ y por ello, los coeficientes que proceden del método clásico que acompañan a $F_{i}^{(k+2)}$ y los introducidos en el nuevo método que acompañan a $F_{i}^{(k)}$ quedan agrupados. Gracias a esto podremos anular el término de error (2.26), que antes era constante y ahora depende de los coeficientes, alcanzando así orden oscilatorio 6.

Veamos con más claridad lo que acabamos de exponer. Para los nuevos esquemas (2.27) y sustituyendo de nuevo (2.22) en las series de Taylor de los errores, los términos en $h^{5}$ y $h^{6}$ son

$$
\begin{array}{r}
\omega^{4} y_{0}^{\prime}\left(\bar{b}_{3} a_{32} c_{2}-\bar{b}_{2}^{*} c_{2} / \omega^{2}-\bar{b}_{3}^{*} c_{3} / \omega^{2}-1 / 120\right) h^{5}, \\
-\omega^{6} y_{0}\left(\bar{b}_{3} a_{32} a_{21}-\bar{b}_{2}^{*} a_{21} / \omega^{2}-\bar{b}_{3}^{*}\left(a_{31}+a_{32}\right) / \omega^{2}-1 / 720\right) h^{6}, \\
-\omega^{6} y_{0}\left(b_{3} a_{32} a_{21}-b_{2}^{*} a_{21} / \omega^{2}-b_{3}^{*}\left(a_{31}+a_{32}\right) / \omega^{2}-1 / 120\right) h^{5}, \\
\omega^{6} y_{0}^{\prime}\left(b_{3}^{*} a_{32} c_{2} / \omega^{2}+1 / 720\right) h^{6} .
\end{array}
$$

Entonces, para conseguir orden oscilatorio 5 será necesario satisfacer, además de (2.6)-(2.16) y (2.28)-(2.30), las siguientes ecuaciones de orden

$$
\begin{aligned}
\bar{b}_{2}^{*} c_{2}+\bar{b}_{3}^{*} c_{3} & =\omega^{2}\left(\bar{b}_{3} a_{32} c_{2}-1 / 120\right), \\
b_{2}^{*} a_{21}+b_{3}^{*}\left(a_{31}+a_{32}\right) & =\omega^{2}\left(b_{3} a_{32} a_{21}-1 / 120\right) .
\end{aligned}
$$

Añadiendo las condiciones (2.17) y (2.18), y (2.19) - (2.21, obtenemos un sistema, que como veremos en la sección siguiente, nos proporcionará una familia biparamétrica de soluciones. Los parámetros libres se pueden elegir para tratar de minimizar el error, consiguiéndose así un método óptimo de orden 5. Resolviendo además de las ecuaciones anteriores, las que siguen

$$
\begin{aligned}
\bar{b}_{2}^{*} a_{21}+\bar{b}_{3}^{*}\left(a_{31}+a_{32}\right) & =\omega^{2}\left(\bar{b}_{3} a_{32} a_{21}-1 / 720\right), \\
b_{3}^{*} c_{2} a_{32} & =-\omega^{2} / 720,
\end{aligned}
$$

obtenemos, como se verá más adelante, un único método de orden oscilatorio 6 . 


\subsubsection{Discusión de métodos de orden oscilatorio cinco}

Como ya hemos visto en la sección anterior, para obtener un método con orden oscilatorio 5, es necesario que los coeficientes de (2.27) satisfagan las ecuaciones (2.6)-(2.16), (2.28)-(2.30), y (2.31) y (2.32). Si añadimos las clásicas condiciones de suma de fila (2.17) y (2.18), y de simplificación de Butcher (2.19) y (2.20) las ecuaciones que se han de verificar son las siguientes:

$$
\begin{aligned}
b_{1}+b_{2}+b_{3} & =1, \\
b_{2} c_{2}+b_{3} c_{3} & =1 / 2, \\
b_{2} c_{2}^{2}+b_{3} c_{3}^{2} & =1 / 3 \\
b_{2} c_{2}^{3}+b_{3} c_{3}^{3} & =1 / 4, \\
b_{3} c_{2} a_{32} & =1 / 24, \\
\bar{b}_{1}^{*}+\bar{b}_{2}^{*}+\bar{b}_{3}^{*} & =0 \\
\bar{b}_{2}^{*} c_{2}+\bar{b}_{3}^{*} c_{3} & =\omega^{2}\left(\bar{b}_{3} a_{32} c_{2}-1 / 120\right), \\
b_{1}^{*}+b_{2}^{*}+b_{3}^{*} & =0 \\
b_{2}^{*} c_{2}+b_{3}^{*} c_{3} & =0, \\
b_{2}^{*} c_{2}^{2}+b_{3}^{*} c_{3}^{2} & =\omega^{2}\left(b_{3} a_{32} c_{2}^{2}-1 / 60\right) .
\end{aligned}
$$

Las cuatro primeras ecuaciones forman un sistema lineal en las variables $b_{i}$, con $i=1,2,3$. Este sistema tiene como matriz asociada

$$
A=\left(\begin{array}{ccc}
1 & 1 & 1 \\
0 & c_{2} & c_{3} \\
0 & c_{2}^{2} & c_{3}^{2} \\
0 & c_{2}^{3} & c_{3}^{3}
\end{array}\right)
$$

y como matriz ampliada

$$
A^{*}=\left(\begin{array}{cccc}
1 & 1 & 1 & 1 \\
0 & c_{2} & c_{3} & 1 / 2 \\
0 & c_{2}^{2} & c_{3}^{2} & 1 / 3 \\
0 & c_{2}^{3} & c_{3}^{3} & 1 / 4
\end{array}\right) .
$$

Según el teorema de Rouché, estos sistemas tendrán solución si coinciden los rangos de la matriz asociada y la matriz ampliada. Vamos a analizar las distintas posibilidades: 
1. Para que el rango de $A$ sea 3 bastará con que el determinante de Vandermonde

$$
c_{2} c_{3}\left|\begin{array}{cc}
1 & 1 \\
c_{2} & c_{3}
\end{array}\right|=c_{2} c_{3}\left(c_{3}-c_{2}\right)
$$

sea distinto de 0 , es decir, que $c_{2} \neq 0$ (esta condición es necesaria además para que se verifique $(2.39)), c_{3} \neq 0$ y $c_{3} \neq c_{2}$. Para que el rango de $A^{*}$ sea también 3 su determinante ha de ser nulo. Esto se cumple si los $c_{i}$ satisfacen:

$$
4\left(c_{2}+c_{3}\right)=3+6 c_{2} c_{3},
$$

es decir,

$$
c_{2}=\frac{4 c_{3}-3}{2\left(3 c_{3}-2\right)}
$$

De esta relación se deduce que $c_{3} \neq 2 / 3$.

Resolviendo el sistema y sustituyendo (2.46), obtenemos los coeficientes $b_{i}$ en función del parámetro $c_{3}$

$$
\begin{aligned}
b_{1} & =\frac{6 c_{3}^{2}-6 c_{3}+1}{6 c_{3}\left(4 c_{3}-3\right)}, \\
b_{2} & =\frac{2\left(9 c_{3}^{2}-12 c_{3}+4\right)\left(3 c_{3}-2\right)}{3\left(6 c_{3}^{2}-8 c_{3}+3\right)\left(4 c_{3}-3\right)}, \\
b_{3} & =\frac{1}{6 c_{3}\left(6 c_{3}^{2}-8 c_{3}+3\right)},
\end{aligned}
$$

Observando estas expresiones se llega a que $c_{3} \neq 3 / 4$. Según (2.39) es necesario que $b_{3} \neq 0$ y a la vista de (2.49), esta condición se satisface para los valores de $c_{3}$ que estamos considerando.

El siguiente paso será obtener $\operatorname{los} \bar{b}_{i}^{*} \mathrm{y} \operatorname{los} b_{i}^{*}$, con $i=1,2,3$. Las ecuaciones (2.40) y (2.41) forman un sistema lineal en las indeterminadas $\bar{b}_{i}^{*}$ y del mismo modo (2.42), (2.43) y (2.44) en las $b_{i}^{*}$. El primer sistema tiene rango 2 y esto da lugar a una familia de soluciones que depende de uno de los coeficientes $\bar{b}_{i}^{*}$ :

$$
\begin{aligned}
\bar{b}_{1}^{*} & =\frac{\left(c_{3}-c_{2}\right) \bar{b}_{3}^{*}-\omega^{2}\left(\bar{b}_{3} a_{32} c_{2}-1 / 120\right)}{c_{2}}, \\
\bar{b}_{2}^{*} & =\frac{\omega^{2}\left(\bar{b}_{3} a_{32} c_{2}-1 / 120\right)-c_{3} \bar{b}_{3}^{*}}{c_{2}}, \\
\bar{b}_{3}^{*} & =\bar{b}_{3}^{*} .
\end{aligned}
$$


El segundo sistema tiene rango 3 y resolviendo obtenemos:

$$
\begin{aligned}
& b_{1}^{*}=\frac{-2 \omega^{2}\left(b_{3} a_{32} a_{21}-1 / 120\right)}{c_{2} c_{3}}, \\
& b_{2}^{*}=\frac{2 \omega^{2}\left(b_{3} a_{32} a_{21}-1 / 120\right)}{c_{2}\left(c_{2}-c_{3}\right)}, \\
& b_{3}^{*}=\frac{2 \omega^{2}\left(b_{3} a_{32} a_{21}-1 / 120\right)}{c_{3}\left(c_{3}-c_{2}\right)} .
\end{aligned}
$$

Los $a_{i j}$ se obtienen a partir de las condiciones de suma de fila (2.17) y (2.18) y la ecuación (2.39) y los $\bar{b}_{i}$ gracias a (2.19) y (2.20). Así tenemos

$$
\begin{aligned}
a_{21} & =\frac{c_{2}^{2}}{2}, \\
a_{31} & =\frac{c_{3}^{2}}{2}-a_{32}, \\
a_{32} & =\frac{1}{24 c_{2} b_{3}} \\
\bar{b}_{1} & =b_{1}, \\
\bar{b}_{2} & =\frac{2-3 c_{3}}{6 c_{2}\left(c_{2}-c_{3}\right)}\left(1-c_{2}\right), \\
\bar{b}_{3} & =\frac{2-3 c_{2}}{6 c_{3}\left(c_{3}-c_{2}\right)}\left(1-c_{3}\right) .
\end{aligned}
$$

Expresando todos los coeficientes en función de $c_{3}$ y $\bar{b}_{3}^{*}$, obtenemos una familia biparamétrica de métodos del tipo (2.27) a partir de los siguientes valores:

$$
\begin{aligned}
& \bar{b}_{1}=\frac{1}{6} \frac{6 \alpha^{2}-6 \alpha+1}{\alpha(4 \alpha-3)}, \quad \bar{b}_{2}=\frac{1}{3} \frac{\left(9 \alpha^{2}-12 \alpha+4\right)(2 \alpha-1)}{\left(6 \alpha^{2}-8 \alpha+3\right)(4 \alpha-3)}, \\
& \bar{b}_{3}=\frac{-1}{6} \frac{\alpha-1}{\alpha\left(6 \alpha^{2}-8 \alpha+3\right)}, \\
& b_{1}=\frac{1}{6} \frac{6 \alpha^{2}-6 \alpha+1}{\alpha(4 \alpha-3)}, \quad b_{2}=\frac{2}{3} \frac{\left(9 \alpha^{2}-12 \alpha+4\right)(3 \alpha-2)}{(4 \alpha-3)\left(6 \alpha^{2}-8 \alpha+3\right)} \\
& b_{3}=\frac{1}{6} \frac{1}{\alpha\left(6 \alpha^{2}-8 \alpha+3\right)}
\end{aligned}
$$




$$
\begin{aligned}
\bar{b}_{1}^{*} & =\frac{1}{60} \frac{360 \alpha^{2} \beta-480 \alpha \beta+\omega^{2}\left(15 \alpha^{2}-22 \alpha+8\right)+180 \beta}{4 \alpha-3}, \\
\bar{b}_{2}^{*} & =\frac{-1}{60} \frac{\left(\omega^{2}(5 \alpha-4)+120 \alpha \beta\right)(3 \alpha-2)}{4 \alpha-3}, \quad \bar{b}_{3}^{*}=\beta \\
b_{1}^{*} & =\frac{1}{120} \frac{\omega^{2}(8 \alpha-7)}{\alpha(4 \alpha-3)}, \quad b_{2}^{*}=\frac{-1}{60} \frac{\omega^{2}(8 \alpha-7)(3 \alpha-2)}{\left(6 \alpha^{2}-8 \alpha+3\right)(4 \alpha-3)}, \\
b_{3}^{*} & =\frac{1}{120} \frac{\omega^{2}(8 \alpha-7)}{\left(6 \alpha^{2}-8 \alpha+3\right)}, \\
a_{21} & =\frac{1}{8}\left(\frac{4 \alpha-3}{3 \alpha-2}\right)^{2}, \quad a_{31}=-\frac{\alpha\left(9 \alpha^{3}-20 \alpha^{2}+14 \alpha-3\right)}{4 \alpha-3} \\
a_{32} & =\frac{1}{2} \frac{\alpha(3 \alpha-2)\left(6 \alpha^{2}-8 \alpha+3\right)}{4 \alpha-3}, \\
c_{2} & =\frac{1}{2} \frac{4 \alpha-3}{3 \alpha-2}, \quad c_{3}=\alpha .
\end{aligned}
$$

siendo $\alpha \neq 3 / 4,2 / 3,0$ y $\beta$ los parámetros. Estos métodos alcanzan orden 5 para el problema no perturbado.

De entre estos métodos mostramos en la Tabla 2.1 los coeficientes del método obtenido con $\alpha=1$ y $\beta=0$.

\begin{tabular}{cc|ccc} 
& 0 & & & $a_{i j}$ \\
$c_{i}$ & $1 / 2$ & $1 / 8$ & & \\
1 & 0 & $1 / 2$ & \\
\hline $\bar{b}_{i}$ & $1 / 6$ & $1 / 3$ & 0 \\
$\bar{b}_{i}^{*}$ & $\omega^{2} / 60$ & $-\omega^{2} / 60$ & 0 \\
\hline$b_{i}$ & $1 / 6$ & $4 / 6$ & $1 / 6$ \\
$b_{i}^{*}$ & $\omega^{2} / 120$ & $-\omega^{2} / 60$ & $\omega^{2} / 120$
\end{tabular}

Tabla 2.1: Un método de orden oscilatorio 5 .

En este caso los coeficientes $b_{i}, \bar{b}_{i}, a_{i j}$ y $c_{i}$ coinciden con los de un método RKN de orden 4 ampliamente conocido (véase Hairer et al. [54]) y además los coeficientes $b_{i}^{*}$ y $\bar{b}_{i}^{*}$ son muy sencillos. 
2. Para que la matriz $A$ tenga rango 2 es preciso que los determinantes de las submatrices $3 \times 3$ obtenidas a partir de ella sean cero. Para ello bastará con que $c_{3}=0$, o bien, $c_{3}=c_{2} \neq 0$. Descartamos la posibilidad de que $c_{2}=0$ porque en ese caso no se satisface la ecuación (2.39). Para que el sistema tenga solución el rango de la matriz $A^{*}$ también tendrá que ser 2 y, por tanto, los determinantes de $A^{*}$ y de las correspondientes matrices $3 \mathrm{x} 3$ deben ser 0 . La primera condición se satisface, como ya hemos visto, si $c_{2}$ y $c_{3}$ cumplen (2.45). Sin embargo, de forma sencilla se puede comprobar que no es posible anular simultáneamente todos los determinantes $3 \times 3$, por lo que podemos concluir que el sistema no tiene solución y los métodos que obtenemos se reducen a los discutidos en el punto 1 .

\subsubsection{Discusión de métodos de orden oscilatorio seis}

De manera análoga a los métodos discutidos con anterioridad, para que el método (2.27) alcance orden oscilatorio 6 es preciso obtener los coeficientes a partir de las ecuaciones (2.6)-(2.16), (2.28)-(2.30), (2.31) y (2.32), y (2.33) y (2.34). Añadiendo las condiciones (2.17) y (2.18), y (2.19) y (2.20), las ecuaciones que se han de verificar son las siguientes:

$$
\begin{aligned}
b_{1}+b_{2}+b_{3} & =1, \\
b_{2} c_{2}+b_{3} c_{3} & =1 / 2, \\
b_{2} c_{2}^{2}+b_{3} c_{3}^{2} & =1 / 3, \\
b_{2} c_{2}^{3}+b_{3} c_{3}^{3} & =1 / 4, \\
b_{3} c_{2} a_{32} & =1 / 24, \\
\bar{b}_{1}^{*}+\bar{b}_{2}^{*}+\bar{b}_{3}^{*} & =0, \\
\bar{b}_{2}^{*} c_{2}+\bar{b}_{3}^{*} c_{3} & =\omega^{2}\left(\bar{b}_{3} a_{32} c_{2}-1 / 120\right), \\
\bar{b}_{2}^{*} c_{2}^{2}+\bar{b}_{3}^{*} c_{3}^{2} & =\omega^{2}\left(\bar{b}_{3} a_{32} c_{2}^{2}-1 / 360\right), \\
b_{1}^{*}+b_{2}^{*}+b_{3}^{*} & =0, \\
b_{2}^{*} c_{2}+b_{3}^{*} c_{3} & =0, \\
b_{2}^{*} c_{2}^{2}+b_{3}^{*} c_{3}^{2} & =\omega^{2}\left(b_{3} a_{32} c_{2}^{2}-1 / 60\right), \\
b_{3}^{*} c_{2} a_{32} & =-\omega^{2} / 720 .
\end{aligned}
$$




\begin{tabular}{cc|ccc} 
& 0 & & & $a_{i j}$ \\
$c_{i}$ & $2 / 9$ & $2 / 81$ & & \\
& $19 / 24$ & $-1235 / 18432$ & $779 / 2048$ & \\
\hline & $\bar{b}_{i}$ & $1 / 76$ & $63 / 164$ & $80 / 779$ \\
$\bar{b}_{i}^{*}$ & $-83 \omega^{2} / 12160$ & $233 \omega^{2} / 26240$ & $-8 \omega^{2} / 3895$ \\
\hline & $1 / 76$ & $81 / 164$ & $384 / 779$ \\
$b_{i}$ & $-4 \omega^{2} / 95$ & $12 \omega^{2} / 205$ & $-64 \omega^{2} / 3895$ \\
$b_{i}^{*}$ & & &
\end{tabular}

Tabla 2.2: Método de orden oscilatorio 6 .

Podemos observar que este sistema de ecuaciones coincide con el obtenido para los métodos de orden oscilatorio 5 añadiéndole las ecuaciones (2.63) y (2.67). Por lo tanto, basta sustituir la solución general biparamétrica anterior en las ecuaciones (2.63) y (2.67), obteniéndose

$$
\begin{gathered}
\frac{2160 \alpha^{3} \beta-(120+2880 \beta) \alpha^{2}+(192+1080 \beta) \alpha-77}{1440(3 \alpha-2)}=0, \\
\frac{\alpha}{60}-\frac{23}{1440}=0,
\end{gathered}
$$

lo que nos lleva a una única solución en la que $\alpha=19 / 4$ y $\beta=$ $-8 \omega^{2} / 3895$. Sustituyendo en el resto de expresiones se obtiene un único método (2.27) cuyos coeficientes son los que se muestran en la Tabla 2.2:

Nota 2.2.1 Como se puede apreciar en las Tablas 2.1 y 2.2 en los coeficientes $b_{i}^{*}$ y $\bar{b}_{i}^{*}$ aparece siempre el factor $\omega^{2}$.

\subsubsection{Experimentos numéricos}

Resulta conveniente en este momento comprobar cómo se comportan los métodos obtenidos con respecto a otros códigos. Para ello consideraremos tres problemas diferentes consistentes en la integración de 


\begin{tabular}{cc|ccc} 
& 0 & & & $a_{i j}$ \\
$c_{i}$ & $1 / 2$ & $1 / 8$ & & \\
1 & 0 & $1 / 2$ & \\
\hline & $\bar{b}_{i}$ & $1 / 6$ & $1 / 3$ & 0 \\
\hline & $b_{i}$ & $1 / 6$ & $4 / 6$ & $1 / 6$
\end{tabular}

Tabla 2.3: Método RKN de orden 4

osciladores o sistemas de osciladores perturbados de la forma (2.1). Compararemos los resultados de los esquemas que hemos construido con los resultados obtenidos con un método RKN muy frecuente en la bibliografía (Hairer et al. [54]), con el método debido a González et al. (véase González [44] y González et al. [47]), que considerábamos en la sección 1.3 y que ha demostrado en experimentos anteriores un buen comportamiento para integrar el tipo de problemas que estamos considerando, y con un código simpléctico desarrollado por Calvo y SanzSerna [7, 97]. Todos estos métodos son explícitos, tienen orden cuatro y excepto el método simpléctico, son códigos de tres etapas. El integrador simpléctico es un esquema RKN de cinco etapas y propiedad FSAL (First Same As Last): sólo necesita cuatro evaluaciones de la función por paso, salvo en el primero.

Los experimentos se han realizado integrando 10 revoluciones para distintos valores para $h$. Los cálculos han sido realizados con precisión aritmética de 16 dígitos.

En las figuras denotaremos por $\mathrm{RKN}^{2} 4: 5$ el método con orden oscilatorio 5 de la Tabla (2.1), por $\mathrm{RKNh}^{2} 4: 6$ el algoritmo de orden oscilatorio 6 de la Tabla (2.2), por RKN4 el método clásico cuyos coeficientes corresponden a la Tabla (2.3), por RKGM4 el método de orden 4 debido a González et al. [44, 47] y por Calvo4 el método simpléctico. Se ha representado el logaritmo decimal del error máximo a lo largo de la integración frente al logaritmo decimal del número de evaluaciones de función empleadas. 
Figura 2.1: Oscilador de Duffing. $\varepsilon=10^{-1}$.

El primer problema que consideramos es el oscilador de Duffing:

$$
y^{\prime \prime}=-y+\varepsilon y^{3},
$$

con condiciones iniciales

$$
y(0)=1, y^{\prime}(0)=0 .
$$

Este problema interviene en la descripción de diversos sistemas mecánicos, tales como el caso del péndulo o de una masa conectada a un muelle que oscila, y está también presente en ciertos sistemas eléctricos.

En las Figuras 2.1 y 2.2 observamos los resultados obtenidos al integrar este problema con los distintos métodos y dos valores del parámetro de perturbación, $\varepsilon=10^{-1}$ y $\varepsilon=10^{-3}$ respectivamente. El error se ha calculado con respecto a una solución de referencia obtenida mediante técnicas de perturbaciones desarrolladas por Farto et al. $[24,25]$, que es extremadamente precisa a largo plazo. En la integración los tamaños de paso se han tomado entre $1 / 2$ y $1 / 2^{7}$ para todos los métodos. 
Figura 2.2: Oscilador de Duffing. $\varepsilon=10^{-3}$.

En ambos experimentos se observa que los resultados de los nuevos métodos son mejores que los del método clásico. En la Figura 2.1 se puede apreciar que el método RKN $h^{2} 4: 6$ es ligeramente mejor que el esquema RKGM4. Para valores de $h$ pequeños apenas se distinguen las gráficas correspondientes a los métodos RKN $h^{2} 4: 5$ y RKGM4 porque presentan un comportamiento muy similar. El método simpléctico proporciona al integrar este problema el mejor comportamiento. Sin embargo, la diferencia entre los métodos crece cuando disminuye el valor de $\varepsilon$. Lógicamente, para problemas suficientemente regulares, cuando $\varepsilon$ es pequeño la solución está mucho más próxima a la solución del problema no perturbado y, por tanto, los nuevos métodos deben proporcionar mejores resultados. El método que mejor se comporta es RKN $h^{2} 4: 6$, seguido muy de cerca por el método RKGM4. El método Calvo4 presenta ahora un comportamiento mucho peor que en el caso anterior, siendo superado por el método RKN $h^{2} 4: 5$ que ni siquiera es el método de orden oscilatorio 5 óptimo. 
El segundo problema que consideraremos es el sistema de osciladores

$$
\begin{aligned}
& y_{1}^{\prime \prime}=-y_{1}+10^{-4}\left(y_{1}^{3}-y_{2}^{2}\right), \\
& y_{2}^{\prime \prime}=-y_{2}+10^{-4}\left(y_{2}^{5}-2 y_{1} y_{2}\right)
\end{aligned}
$$

con condiciones iniciales

$$
y_{1}(0)=y_{2}(0)=0, y_{1}^{\prime}(0)=y_{2}^{\prime}(0)=1 \text {. }
$$

La Figura 2.3 muestra los resultados obtenidos al integrar este problema.

Figura 2.3: Sistema de osciladores.

Los errores han sido calculados con respecto a la integral primera $H\left(y_{1}, y_{1}^{\prime}, y_{2}, y_{2}^{\prime}\right)=\frac{1}{2}\left(y_{1}^{2}+y_{1}^{\prime 2}+y_{2}^{2}+y_{2}^{\prime 2}\right)-\frac{10^{-4}}{4} y_{1}^{4}+10^{-4} y_{1} y_{2}^{2}-\frac{10^{-4}}{6} y_{2}^{6}$.

Los tamaños de paso considerados oscilan entre $1 / 2$ y $1 / 2^{7}$ para el método RKN4, $1 / 2$ y $1 / 2^{8}$ para el código RKN $h^{2} 4: 5$, y $1 / 2$ y $1 / 2^{6}$ 
para los restantes. En este ejemplo se puede ver el excelente comportamiento del método RKN $h^{2} 4: 6$. Para los valores de $h$ más pequeños proporciona errores menores que el código RKGM4, aunque éste se muestre más eficiente para los valores de $h$ más grandes. El método RKN $h^{2} 4: 5$ para este problema se comporta peor, aunque en cualquier caso, proporciona soluciones más aproximadas que el método clásico y para $h$ pequeño es más eficiente que el método simpléctico.

Como último ejemplo consideraremos el problema de la predicción de la órbita de un satélite artificial. Hemos tomado las ecuaciones de movimiento formuladas en variables focales (véase Ferrándiz [29] y Ferrándiz et al. [34]). En dichas variables el problema se reduce a un sistema de cuatro osciladores perturbados con frecuencia unidad

$$
\begin{aligned}
& y_{i}^{\prime \prime}=-y_{i}+Q_{i}, \quad i=1,2,3, \\
& u^{\prime \prime}=-u+\frac{\mu}{c^{2}}+Q
\end{aligned}
$$

donde $\mu$ es la masa reducida y $Q_{i}$ y $Q$ son los correspondientes términos de perturbación. En los experimentos que se desarrollarán a lo largo de la memoria se ha tomado como perturbación sólamente la debida al achatamiento terrestre, es decir, consideraremos solamente el armónico zonal $J_{2}$. Además se ha considerado una órbita ecuatorial. De esta forma las ecuaciones anteriores se concretan en

$$
\begin{aligned}
& y_{i}^{\prime \prime}=-y_{i}, \quad i=1,2,3, \\
& u^{\prime \prime}=-u+\frac{\mu}{c^{2}}+12 \frac{J_{2}}{c^{2}} u^{2},
\end{aligned}
$$

donde $c$ es el momento angular. Hemos usado como solución de referencia la obtenida por Farto et al. [24]. La integración se ha realizado para valores de $h$ variando entre $1 / 2$ y $1 / 2^{7}$. En la Figura 2.4 se muestran los resultados de integrar una órbita circular $(e=0)$.

Los nuevos métodos vuelven a mostrar su buen comportamiento en la integración de este tipo de problemas. Fijado un error se necesitan menos evaluaciones de función con los métodos que presentamos que con los códigos clásico y simpléctico. Comparados con el algoritmo RKGM4, el método RKN $h^{2}$ 4:6 también requiere menos evaluaciones de función por paso.

Para terminar, mostramos el mismo experimento (Figura 2.5) con una órbita altamente excéntrica $(e=0.99)$. Los resultados son similares a los del caso anterior. Como se puede apreciar el método 
Figura 2.4: Problema del satélite. $e=0$.

$\mathrm{RKN} h^{2} 4: 5$, para los valores de $h$ menores, tiene un comportamiento muy similar al código RKGM4.

\subsection{Métodos RKN $h^{2}$. Propiedades}

\subsubsection{Formulación general}

En esta sección intentaremos formalizar las ideas que nos han conducido a la construcción de unos nuevos métodos que parecen presentar un excelente comportamiento en la integración de osciladores perturbados. En todos los métodos discutidos se observa que los nuevos coeficientes $\bar{b}_{i}^{*}$ y $b_{i}^{*}$ son siempre constantes multiplicadas por $\omega^{2}$. Esto nos ha llevado a reformular los coeficientes para facilitar el manejo y a partir de este momento denotaremos por $\omega^{2} \bar{b}_{i}^{*}$ y $\omega^{2} b_{i}^{*}$ a los antiguos coeficientes $\bar{b}_{i}^{*}$ y $b_{i}^{*}$ de los métodos que estamos desarrollando.

Definición 2.3.1 Sean $s \in \mathbb{N}, a_{i j} i=2, \ldots, s, j=1, \ldots, i-1$, 
Figura 2.5: Problema del satélite. $e=0.99$.

$b_{i}, b_{i}^{*}, \bar{b}_{i}, \bar{b}_{i}^{*}, i=1, \ldots, s, c_{1}=0$ y $c_{i} i=2, \ldots, s$ coeficientes reales. Llamaremos método $\mathrm{RKN} h^{2}$ explícito de $s$ etapas para el problema (2.2) al siguiente esquema

$$
\begin{aligned}
& k_{i}=f\left(x_{0}+c_{i} h, y_{0}+h c_{i} y_{0}^{\prime}+h^{2} \sum_{j=1}^{i-1} a_{i j} k_{j}\right), \quad i=1, \ldots, s, \\
& y_{1}=y_{0}+h y_{0}^{\prime}+h^{2} \sum_{i=1}^{s}\left(\bar{b}_{i}+h^{2} \omega^{2} \bar{b}_{i}^{*}\right) k_{i}, \\
& y_{1}^{\prime}=y_{0}^{\prime}+h \sum_{i=1}^{s}\left(b_{i}+h^{2} \omega^{2} b_{i}^{*}\right) k_{i} .
\end{aligned}
$$

Nota 2.3.2 Aunque nos referiremos a los anteriores como métodos $\mathrm{RKN} h^{2}$, en realidad son una familia de métodos, ya que para cada frecuencia considerada tendremos un código particular. 
Nota 2.3.3 En esta memoria sólo estudiaremos los métodos explícitos, es decir, el caso en que $c_{1}=0$ y $a_{i j}=0$ si $i \leq j$.

Nota 2.3.4 Igual que los métodos RKN, (véase Lambert [69]) los códigos que acabamos de definir se pueden utilizar para integrar sistemas de ecuaciones.

Definición 2.3.5 Los métodos $\mathrm{RKN}^{2}$ son de orden $p$ si para funciones $f$ suficientemente regulares se cumple

$$
\begin{aligned}
y\left(x_{0}+h\right)-y_{1} & =O\left(h^{p+1}\right), \\
y^{\prime}\left(x_{0}+h\right)-y_{1}^{\prime} & =O\left(h^{p+1}\right) .
\end{aligned}
$$

\subsubsection{Error de truncación local}

Consideremos un método numérico de un paso para el problema (2.2) que podemos escribir como sigue

$$
\begin{aligned}
& y_{n+1}=y_{n}+h \phi\left(x_{n}, y_{n}, y_{n}^{\prime}, h\right), \\
& y_{n+1}^{\prime}=y_{n}^{\prime}+h \phi^{\prime}\left(x_{n}, y_{n}, y_{n}^{\prime}, h\right),
\end{aligned}
$$

donde, según la notación de Henrici [57], $\phi$ y $\phi^{\prime}$ son las funciones incremento del método. Los métodos $\mathrm{RKN} h^{2}$ se pueden escribir de esta forma considerando

$$
\begin{aligned}
\phi & =y^{\prime}+h \sum_{i=1}^{s}\left(\bar{b}_{i}+h^{2} \omega^{2} \bar{b}_{i}^{*}\right) k_{i}, \\
\phi^{\prime} & =\sum_{i=1}^{s}\left(b_{i}+h^{2} \omega^{2} b_{i}^{*}\right) k_{i},
\end{aligned}
$$

de manera similar a un método RKN (véase Dormand [19] y Dormand et al. $[20,21])$. Volviendo sobre (2.70), podemos reescribirlo como dos ecuaciones

$$
\begin{aligned}
& 0=y_{n}+h \phi\left(x_{n}, y_{n}, y_{n}^{\prime}, h\right)-y_{n+1} \\
& 0=y_{n}^{\prime}+h \phi^{\prime}\left(x_{n}, y_{n}, y_{n}^{\prime}, h\right)-y_{n+1}^{\prime}
\end{aligned}
$$


que en general no se satisfacen si se sustituye la solución exacta $y\left(x_{n}\right)$ por $y_{n}$, y su derivada $y^{\prime}\left(x_{n}\right)$ por $y_{n}^{\prime}$. La discrepancia se define como error de truncación local

$$
\begin{aligned}
& t_{n+1}=y\left(x_{n}\right)+h \phi\left(x_{n}, y\left(x_{n}\right), y^{\prime}\left(x_{n}\right), h\right)-y\left(x_{n+1}\right), \\
& t_{n+1}^{\prime}=y^{\prime}\left(x_{n}\right)+h \phi\left(x_{n}, y\left(x_{n}\right), y^{\prime}\left(x_{n}\right), h\right)-y^{\prime}\left(x_{n+1}\right),
\end{aligned}
$$

y corresponde a la cantidad por la cual la solución exacta no satisface la fórmula numérica. No obstante una cantidad más práctica desde el punto de vista computacional, es el error por paso o error local, que se calcula como la diferencia $y\left(x_{n+1}\right)-y_{n+1}$, o bien $y^{\prime}\left(x_{n+1}\right)-y_{n+1}^{\prime}$, considerando que los valores anteriores son exactos. Afortunadamente los dos errores, local y de truncación local, son muy similares en magnitud (véase Dormand [19]) y por ello unas buenas estimaciones (tanto mejor cuanto menor sea el tamaño de paso $h$ ) de los errores de truncación local serán

$$
\begin{aligned}
& y_{1}-y\left(x_{0}+h\right), \\
& y_{1}^{\prime}-y^{\prime}\left(x_{0}+h\right) .
\end{aligned}
$$

La solución exacta y su derivada se pueden expresar según su desarrollo de Taylor

$$
\begin{aligned}
& y\left(x_{0}+h\right)=y_{0}+h y_{0}^{\prime}+h^{2} \sum_{i=2}^{\infty} \frac{h^{i-2}}{i !} f^{(i-2)}\left(x_{0}, y_{0}\right) \\
& y^{\prime}\left(x_{0}+h\right)=y_{0}^{\prime}+h \sum_{i=1}^{\infty} \frac{h^{i-1}}{i !} f^{(i-1)}\left(x_{0}, y_{0}\right) .
\end{aligned}
$$

que en términos de las diferenciales elementales se pueden escribir

$$
\begin{aligned}
& y\left(x_{0}+h\right)=y_{0}+h y_{0}^{\prime}+h^{2} \sum_{i=2}^{\infty} h^{i-2} \sum_{j=1}^{n_{i}} \frac{\alpha_{j}^{(i)}}{i !} F_{j}^{(i)}\left(x_{0}, y_{0}, y_{0}^{\prime}\right), \\
& y^{\prime}\left(x_{0}+h\right)=y_{0}^{\prime}+h \sum_{i=1}^{\infty} h^{i-1} \sum_{j=1}^{n_{i+1}} \frac{\alpha_{j}^{(i+1)}}{i !} F_{j}^{(i+1)}\left(x_{0}, y_{0}, y_{0}^{\prime}\right),
\end{aligned}
$$

donde $\alpha_{j}^{(k)}$ es el coeficiente que acompaña a $F_{j}^{(k)}$ en $f^{(k)}$. Para abreviar la notación en lo sucesivo representamos $F_{j}^{(i)}\left(x_{0}, y_{0}, y_{0}^{\prime}\right)$ simplemente como $F_{j}^{(i)}$. 
Desarrollando también en serie de Taylor las aproximaciones numéricas en torno a $h=0$ se tiene

$$
\begin{aligned}
y_{1} & =y_{0}+h y_{0}^{\prime}+h^{2} \sum_{i=2}^{\infty} h^{i-2}\left\{\sum_{j=1}^{n_{i}} \bar{\beta}_{j}^{(i)} F_{j}^{(i)}\right\} \\
& +\omega^{2} h^{4} \sum_{i=2}^{\infty} h^{i-2}\left\{\sum_{j=1}^{n_{i}} \bar{\beta}_{j}^{*(i)} F_{j}^{(i)}\right\} \\
y_{1}^{\prime} & =y_{0}^{\prime}+h \sum_{i=1}^{\infty} h^{i-1}\left\{\sum_{j=1}^{n_{i+1}} \beta_{j}^{(i)} F_{j}^{(i+1)}\right\} \\
& +\omega^{2} h^{3} \sum_{i=1}^{\infty} h^{i-1}\left\{\sum_{j=1}^{n_{i+1}} \beta_{j}^{*(i)} F_{j}^{(i+1)}\right\},
\end{aligned}
$$

donde $\bar{\beta}_{j}^{(i)}$ y $\beta_{j}^{(i)}$ son funciones de los parámetros $a_{i j}, c_{i}$ y $\bar{b}_{i}$ ó $b_{i}$ respectivamente, y $\bar{\beta}_{j}^{*(i)}$ y $\beta_{j}^{*(i)}$ son idénticas pero sustituyendo $\bar{b}_{i}$ por $\bar{b}_{i}^{*}$ y $b_{i}$ por $b_{i}^{*}$. Con $n_{i}$ representamos el número de diferenciales elementales de orden $i$.

Entonces,

$$
\begin{aligned}
y_{1}-y\left(x_{0}+h\right) & =\sum_{i=2}^{\infty} h^{i}\left\{\sum_{j=1}^{n_{i}}\left(\bar{\beta}_{j}^{(i)}-\frac{\alpha_{j}^{(i)}}{i !}\right) F_{j}^{(i)}\right\} \\
& +\omega^{2} \sum_{i=4}^{\infty} h^{i}\left\{\sum_{j=1}^{n_{i-2}} \bar{\beta}_{j}^{*(i-2)} F_{j}^{(i-2)}\right\}, \\
y_{1}^{\prime}-y^{\prime}\left(x_{0}+h\right) & =\sum_{i=2}^{\infty} h^{i-1}\left\{\sum_{j=1}^{n_{i}}\left(\beta_{j}^{(i-1)}-\frac{\alpha_{j}^{(i-1)}}{(i-1) !}\right) F_{j}^{(i)}\right\} \\
& +\omega^{2} \sum_{i=4}^{\infty} h^{i-1}\left\{\sum_{j=1}^{n_{i-2}} \beta_{j}^{*(i-3)} F_{j}^{(i-2)}\right\} .
\end{aligned}
$$

En ambas expresiones los primeros sumatorios coinciden con los errores locales para los métodos RKN (véase Dormand [19]).

Definiendo los coeficientes de error

$$
\begin{aligned}
& \tau_{j}^{(i)}=\bar{\beta}_{j}^{(i)}-\frac{\alpha_{j}^{(i)}}{i !}, \quad \tau_{j}^{*(i)}=\bar{\beta}_{j}^{*(i)}, \\
& \tau_{j}^{\prime(i)}=\beta_{j}^{(i)}-\frac{\alpha_{j}^{(i)}}{i !}, \quad \tau_{j}^{*(i)}=\beta_{j}^{*(i)},
\end{aligned}
$$


y tomando $\tau_{j}^{*(0)}=\tau_{j}^{*(1)}=0$ y $\tau_{j}^{\prime *(-1)}=\tau_{j}^{*(0)}=0$, tenemos finalmente

$$
\begin{aligned}
& y_{1}-y\left(x_{0}+h\right)=\sum_{i=2}^{\infty} h^{i}\left\{\sum_{j=1}^{n_{i}} \tau_{j}^{(i)} F_{j}^{(i)}+\omega^{2} \sum_{j=1}^{n_{i-2}} \tau_{j}^{*(i-2)} F_{j}^{(i-2)}\right\}, \\
& y_{1}^{\prime}-y^{\prime}\left(x_{0}+h\right)=\sum_{i=1}^{\infty} h^{i}\left\{\sum_{j=1}^{n_{i+1}} \tau_{j}^{(i)} F_{j}^{(i+1)}+\omega^{2} \sum_{j=1}^{n_{i-1}} \tau_{j}^{*^{\prime}(i-2)} F_{j}^{(i-1)}\right\} .
\end{aligned}
$$

En la Tabla 2.4 tenemos los coeficientes de error $\tau_{j}^{(i)}$ para los primeros valores de $i$. Para obtener los correspondientes a la derivada basta sustituir $\tau_{j}^{(i)}$ por $\tau_{j}^{(i-1)}, \bar{b}_{i}$ por $b_{i}$ y multiplicar cada fracción a la derecha del signo menos por $i$. Los coeficientes $\tau_{j}^{*(i)}$ y $\tau_{j}^{*(i)}$, siguiendo la definición, se obtienen de los correspondientes $\tau_{j}^{(i)}$ y $\tau_{j}^{\left({ }^{(i)}\right.}$ sustituyendo $\bar{b}_{i}$ por $\bar{b}_{i}^{*}$ y $b_{i}$ por $b_{i}^{*}$ y eliminando las fracciones a la derecha del signo menos.

Vamos a particularizar ahora las anteriores expresiones del error de truncación local para los dos problemas que centran nuestra atención a lo largo de esta memoria.

En primer lugar consideremos el caso del oscilador no perturbado. En este caso la función a considerar es $f=-\omega^{2} y$ y para cada $i$ sólo resulta una diferencial elemental no nula que denotaremos por $F_{o s c}^{(i)}$. Como ya veíamos en $(2.22)$

$$
F_{\text {osc }}^{(i)}=\left\{\begin{array}{lll}
(-1)^{n} \omega^{2 n} y & \text { si } & i=2 n \\
(-1)^{n} \omega^{2 n} y^{\prime} & \text { si } & i=2 n+1
\end{array}\right.
$$

De forma sencilla se observa que

$$
F_{o s c}^{(i)}=-\omega^{2} F_{o s c}^{(i-2)}
$$

y de este modo para los métodos $\mathrm{RKN} h^{2}$ la expresión del error local se simplifica notablemente

$$
\begin{aligned}
& y_{1}-y\left(x_{0}+h\right)=\sum_{i=2}^{\infty} h^{i}\left(\tau^{(i)}-\omega^{2} \frac{\tau^{*(i-2)}}{\omega^{2}}\right) F_{o s c}^{(i)}, \\
& y_{1}^{\prime}-y^{\prime}\left(x_{0}+h\right)=\sum_{i=1}^{\infty} h^{i}\left(\tau^{(i)^{\prime}}-\omega^{2} \frac{\tau^{*(i-2)}}{\omega^{2}}\right) F_{o s c}^{(i+1)},
\end{aligned}
$$




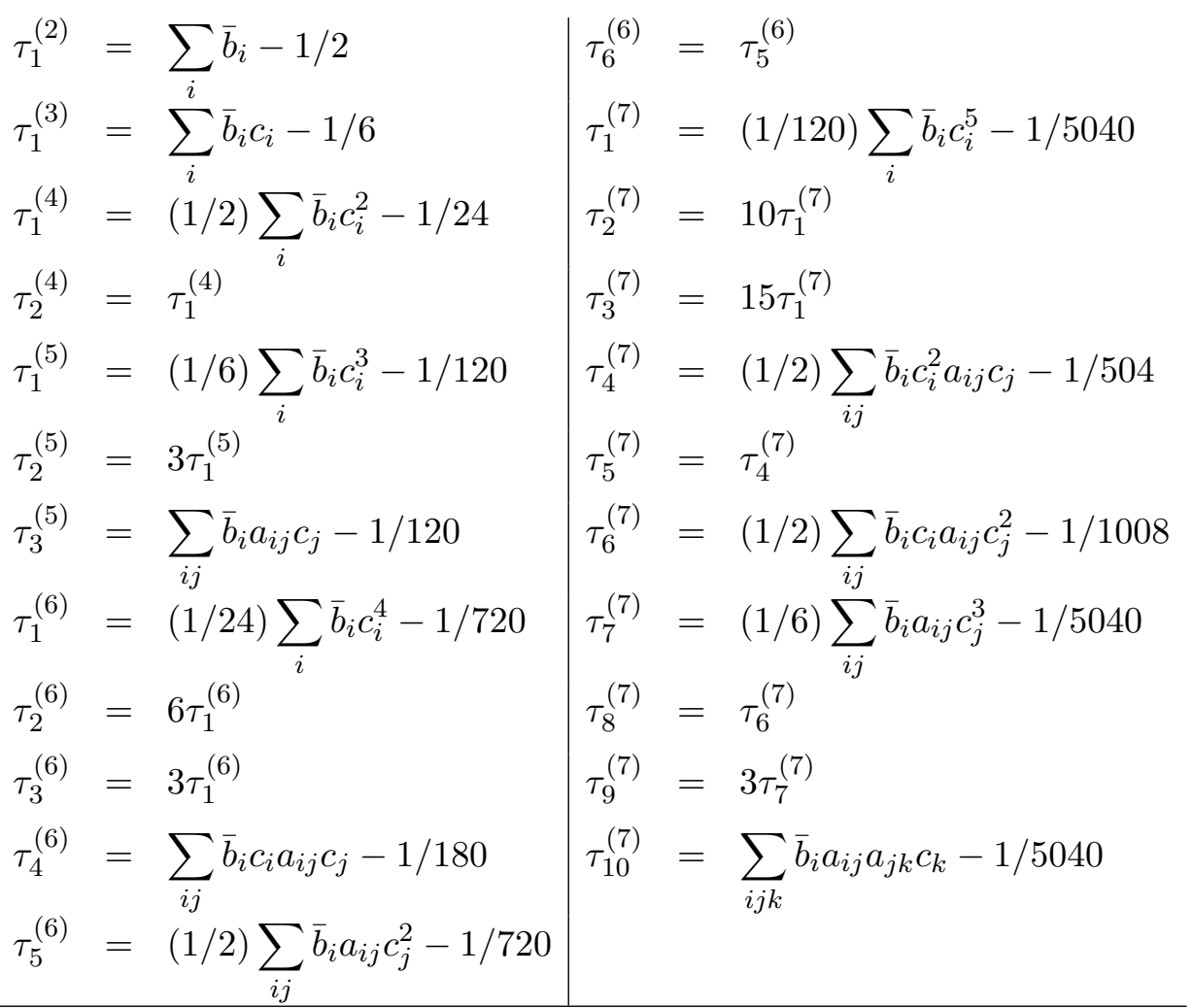

Tabla 2.4: Coeficientes de error 
donde designaremos por $\tau^{(i)}, \tau^{*(i)}, \tau^{\prime(i)}$ y $\tau^{\prime *(i)}$ los coeficientes de error que acompañan a la correspondiente diferencial elemental no nula. A la vista de las expresiones anteriores, podemos observar que multiplicando a las diferenciales elementales aparece un factor que consta de dos sumandos. En el caso de un RKN en este factor aparece sólo el primer sumando. Nosotros aprovecharemos el segundo sumando para intentar elevar el orden de un método $\mathrm{RKN} h^{2}$ para el problema no perturbado (como hicimos en las secciones precedentes), ya que fijado un número de etapas $s$ contamos con un mayor número de coeficientes de error que dependen de los parámetros del método.

Consideremos ahora el caso del oscilador perturbado. En este caso la función del problema $(2.2)$ es $f=-\omega^{2} y+\varepsilon g(x, y)$, entonces, los errores locales para un método $\mathrm{RKN} h^{2}$ vienen dados por

$$
\begin{aligned}
y_{1}-y\left(x_{0}+h\right) & =\sum_{i=2}^{\infty} h^{i}\left(\tau^{(i)}-\tau^{*(i-2)}\right) F_{o s c}^{(i)} \\
& +\varepsilon \sum_{i=2}^{\infty} h^{i}\left\{\sum_{j=1}^{n_{i}} \tau_{j}^{(i)} G_{j}^{(i)}+\omega^{2} \sum_{j=1}^{n_{i-2}} \tau_{j}^{*(i-2)} G_{j}^{(i-2)}\right\} \\
y_{1}^{\prime}-y^{\prime}\left(x_{0}+h\right)= & \sum_{i=1}^{\infty} h^{i}\left(\tau^{\prime}(i)-\tau^{\prime *(i-2)}\right) F_{o s c}^{(i+1)} \\
& +\varepsilon \sum_{i=1}^{\infty} h^{i}\left\{\sum_{j=1}^{n_{i+1}} \tau_{j}^{\prime(i)} G_{j}^{(i+1)}+\omega^{2} \sum_{j=1}^{n_{i-1}} \tau_{j}^{*(i-2)} G_{j}^{(i-1)}\right\}
\end{aligned}
$$

donde denotamos por $G_{j}^{(i)}$ las diferenciales elementales de la función $g$ y por $F_{o s c}^{(i)}$ las correspondientes a la parte no perturbada de $f$. Lógicamente los primeros sumatorios en ambas expresiones coinciden con los errores locales para el oscilador no perturbado (2.71) y (2.72). 


\subsubsection{Condiciones de orden para un método RKN $h^{2} p: q$}

Según la Definición 2.3.5 para obtener métodos $\mathrm{RKN} h^{2}$ de orden $p$ es necesario

$$
\begin{aligned}
& \tau_{j}^{(i)}=0\left\{\begin{array}{l}
i=2, \ldots, p \\
j=1, \ldots, n_{i}
\end{array} \tau_{j}^{\prime(i)}=0\left\{\begin{array}{l}
i=1, \ldots, p \\
j=1, \ldots, n_{i+1}
\end{array}\right.\right. \\
& \tau_{j}^{*(i)}=0\left\{\begin{array}{l}
i=2, \ldots, p-2 \\
j=1, \ldots, n_{i}
\end{array} \tau_{j}^{*(i)}=0\left\{\begin{array}{l}
i=1, \ldots, p-2 \\
j=1, \ldots, n_{i+1}
\end{array}\right.\right.
\end{aligned}
$$

Este conjunto de ecuaciones constituyen lo que se conoce como condiciones de orden.

Teniendo en cuenta (2.71) y (2.72), las condiciones de orden para un método $\mathrm{RKN} h^{2}$ de orden oscilatorio $q$ se concretan en

$$
\begin{gathered}
\tau^{(i)}-\tau^{*(i-2)}=0, \\
\tau^{\prime(i)}-\tau^{*(i-2)}=0,
\end{gathered}
$$

con $i=1, \ldots, q$.

Como ya mencionamos anteriormente, los coeficientes de error en $y$ e $y^{\prime}$ que dependen de $b_{i}$ y $\bar{b}_{i}$ se hallan relacionados (véase Dormand [19]) y basta conocer los de $y^{\prime}$. En cuanto a los que dependen de los coeficientes $\bar{b}_{i}^{*}$ y $b_{i}^{*}$ se pueden obtener de forma sencilla a partir de los anteriores como ya hemos visto. Pese a estas relaciones, la obtención de los coeficientes de error suele ser muy compleja, por lo que para obtener las condiciones de orden para métodos de orden elevado recurriremos a la conocida teoría de árboles especiales de Nyström con raíz. Dicha teoría es debida a Hairer y Wanner (véase Hairer et al. $[54,55]$ ), que continuaron los trabajos iniciados por Butcher (véase Butcher [6]) para ecuaciones de primer orden. Aunque en este momento no vamos a tratar la teoría de árboles, la adaptaremos más adelante a los métodos que venimos considerando.

Definición 2.3.6 Llamamos método $\mathrm{RKN}^{2} p: q$ a un código $\mathrm{RKN}^{2}$ con orden $p$ para un problema general y orden oscilatorio $q$.

Para obtener un método RKN $h^{2} p: q$ se han de satisfacer las condiciones de orden (2.75) para alcanzar orden $p$ para un problema general y, las condiciones $(2.76)$ y $(2.77)$ con $i=p+1, \ldots, q$, para conseguir orden oscilatorio $q$. 


\subsection{Método $\mathrm{RKN} h^{2} 4: 5$ óptimo}

Como ya hemos mencionado a lo largo de discusiones anteriores, el error de truncación local en la aproximación a la solución para un método RKN de orden $p$ se puede escribir (veáse Dormand [19]) como

$$
t_{n+1}=\sum_{i=p+1}^{\infty} h^{i}\left\{\sum_{j=1}^{n_{i}} \tau_{j}^{(i)} F_{j}^{(i)}\right\}
$$

y análogamente en la aproximación a la derivada

$$
t_{n+1}^{\prime}=\sum_{i=p+1}^{\infty} h^{i}\left\{\sum_{j=1}^{n_{i+1}} \tau_{j}^{\prime(i)} F_{j}^{(i+1)}\right\},
$$

donde $F_{j}^{(i)}$ son las anteriormente mencionadas diferenciales elementales $(2.5)$, y $\tau_{j}^{(i)}$ y $\tau_{j}^{(i)^{\prime}}$ son los correspondientes coeficientes de error para $y$ e $y^{\prime}$.

La influencia dominante en el error de truncación local si el tamaño de paso $h$ es suficientemente pequeño, viene dada por las funciones principales de error:

$$
\varphi_{p}=\sum_{j=1}^{n_{p+1}} \tau_{j}^{(p+1)} F_{j}^{(p+1)}
$$

para el error en la aproximación a la solución y

$$
\varphi_{p}^{\prime}=\sum_{j=1}^{n_{p+2}} \tau_{j}^{(p+1)} F_{j}^{(p+2)}
$$

para en el error en la derivada.

Cuando se tiene una familia de métodos con parámetros libres, éstos se pueden utilizar para obtener métodos óptimos, es decir, fijado un orden obtener el método que consiga minimizar la norma euclídea de los coeficientes de error en las funciones principales de error (Dormand [19]). Se trata por tanto de minimizar la función

$$
\Psi=\sqrt{\left(A^{(p+1)}\right)^{2}+\left(A^{\prime(p+1)}\right)^{2}},
$$


donde

$$
A^{(p+1)}=\sqrt{\sum_{j=1}^{n_{p+1}}\left(\tau_{j}^{(p+1)}\right)^{2}}, \quad A^{\prime(p+1)}=\sqrt{\sum_{j=1}^{n_{p+2}}\left(\tau_{j}^{\prime(p+1)}\right)^{2}} .
$$

Una idea similar utilizaremos en la presente sección para obtener un método $\mathrm{RKN} h^{2}$, como el discutido en la sección 2.2.1, que alcance orden oscilatorio 5 y sea óptimo en el sentido comentado. Recordemos que existía un único método $\mathrm{RKN} h^{2} 4: 6$ de tres etapas y por tanto, dicho esquema es el método óptimo.

La familia de métodos $\mathrm{RKN}^{2}$ que estamos considerando alcanza orden 4 cuando se trata de integrar un problema general y orden oscilatorio 5. Por tanto, las expresiones de los errores locales se obtienen a partir de (2.73) y (2.74) imponiendo orden 5 para la parte no perturbada y orden 4 para la parte perturbada de $f$, es decir,

$$
\begin{aligned}
y_{1}-y\left(x_{0}+h\right) & =\sum_{i=6}^{\infty} h^{i}\left(\tau^{(i)}-\tau^{*(i-2)}\right) F_{o s c}^{(i)} \\
& +\varepsilon \sum_{i=5}^{\infty} h^{i}\left\{\sum_{j=1}^{n_{i}} \tau_{j}^{(i)} G_{j}^{(i)}+\omega^{2} \sum_{j=1}^{n_{i-2}} \tau_{j}^{*(i-2)} G_{j}^{(i-2)}\right\} \\
y_{1}^{\prime}-y^{\prime}\left(x_{0}+h\right) & =\sum_{i=6}^{\infty} h^{i}\left(\tau^{\prime(i)}-\tau^{\prime *(i-2)}\right) F_{o s c}^{(i+1)} \\
& +\varepsilon \sum_{i=5}^{\infty} h^{i}\left\{\sum_{j=1}^{n_{i+1}} \tau_{j}^{(i)} G_{j}^{(i+1)}+\omega^{2} \sum_{j=1}^{n_{i-1}} \tau_{j}^{*(i-2)} G_{j}^{(i-1)}\right\}
\end{aligned}
$$

Observando (2.78) y (2.79), vemos que el término que predomina en el error de truncación local dependerá de los valores de $h$ y $\varepsilon$. Los términos en $h^{5}$ aparecen multiplicados por $\varepsilon$ y por tanto no en todos los casos es posible despreciar los términos en $h^{6}$, dependerá de si $h$ es mayor o menor que $\varepsilon$. Entonces en cada caso concreto convendría minimizar un término u otro dependiendo de en cuál de las situaciones mencionadas nos encontremos. Esto en principio nos llevaría a obtener dos métodos distintos que podríamos seleccionar según el problema. Sin embargo, en una implementación en paso variable el tamaño de paso puede variar notablemente y podemos pasar de una situación a otra numerosas veces a lo largo de la integración. Por ello, una solución de compromiso es tratar de minimizar simultáneamente 
ambos términos de error. Entonces, el método óptimo será aquel que haga mínima la función

$$
\Psi=\sqrt{\left(A^{(5)}\right)^{2}+\left(A^{\prime(5)}\right)^{2}+\left(A^{(6)}\right)^{2}+\left(A^{(6)}\right)^{2}},
$$

donde

$$
\begin{array}{ll}
A^{(5)}=\sqrt{\sum_{j=1}^{3}\left(\tau_{j}^{(5)}\right)^{2}+\left(\tau_{j}^{*(3)}\right)^{2}}, & A^{\prime(5)}=\sqrt{\sum_{j=1}^{6}\left(\tau_{j}^{\prime(5)}\right)^{2}+\left(\tau_{j}^{*(1)}\right)^{2}} \\
A^{(6)}=\sqrt{\left(\tau^{(6)}-\tau^{*(4)}\right)^{2}}, & A^{\prime(6)}=\sqrt{\left(\tau^{\prime}(6)-\tau^{\prime *(4)}\right)^{2}}
\end{array}
$$

Los coeficientes $\tau_{j}^{(5)}, \tau_{j}^{\prime(5)}, \tau_{j}^{*(3)}$ y $\tau_{j}^{\prime *(1)}$ se pueden obtener de forma sencilla a partir de Tabla 2.4. Los coeficientes $\tau^{(6)}, \tau^{\prime(6)}, \tau^{\prime *(4)}$ y $\tau_{j}^{\prime *(4)}$ son los correspondientes coeficientes de error no nulos para el caso concreto del oscilador no perturbado. Si a los métodos $\mathrm{RKN} h^{2}$ correspondientes al esquema (2.27) les exigimos orden oscilatorio 5 tenemos, como ya discutimos en la sección 2.2.1, una familia biparámetrica de códigos dependientes de los parámetros $\alpha=c_{3} \mathrm{y} \beta=\bar{b}_{3}^{*}$. Tras sustituir los coeficientes del método se obtienen:

$$
\begin{aligned}
& \tau_{1}^{(5)}=\frac{-1}{720} \frac{10 \alpha^{2}-12 \alpha+3}{3 \alpha-2}, \quad \tau_{2}^{(5)}=3 \tau_{1}^{(5)}, \\
& \tau_{3}^{(5)}=\frac{1}{30}-\frac{\alpha}{24}, \quad \tau_{1}^{*(3)}=\tau_{3}^{(5)}, \\
& \tau_{1}^{\prime(5)}=\frac{-1}{4} \tau_{1}^{(5)}, \quad \tau_{2}^{\prime(5)}=\frac{-3}{2} \tau_{1}^{(5)}, \\
& \tau_{3}^{\prime(5)}=\frac{-3}{4} \tau_{1}^{(5)}, \quad \tau_{4}^{\prime(5)}=-\tau_{3}^{(5)}, \\
& \tau_{5}^{\prime(5)}=\frac{1}{480} \frac{8 \alpha-7}{3 \alpha-2}, \quad \tau_{6}^{\prime(5)}=\tau_{5}^{\prime(5)}, \\
& \tau_{1}^{*(3)}=\tau_{5}^{\prime(5)} \quad \tau_{2}^{\prime *(3)}=\tau_{5}^{\prime(5)} .
\end{aligned}
$$

Los términos en $h^{6}$ en los errores locales son

$$
\begin{array}{r}
-h^{6}\left(\bar{b}_{3} a_{32} a_{21}-\frac{1}{720}-\left(\bar{b}_{2}^{*} a_{21}+\bar{b}_{3}^{*}\left(a_{31}+a_{32}\right)\right) \omega^{6} y_{0}\right. \\
h^{6}\left(b_{3}^{*} a_{32} c_{2}+\frac{1}{720}\right) \omega^{6} y_{0}^{\prime}
\end{array}
$$


y nos permiten obtener $\tau^{(6)}-\tau^{*(4)}$ y $\tau^{\prime(6)}-\tau^{\prime *(4)}$. Podemos expresarlos de nuevo en función de los parámetros del método

$$
\begin{aligned}
& \tau^{(6)}-\tau^{*(4)}=\frac{\alpha}{60}-\frac{19}{1440}, \\
& \tau^{\prime(6)}-\tau^{*(3)}= \\
& \frac{\alpha \beta\left(2160 \alpha^{3}-6660 \alpha^{2}+6120 \alpha-1890\right)+\alpha(60 \alpha-99)+40}{1440(3 \alpha-2)} .
\end{aligned}
$$

Sustituyendo los coeficientes de error en (2.80) se obtiene una función en los parámetros $\alpha$ y $\beta$ que buscamos minimizar

$$
\sqrt{\frac{103}{8}\left(\tau_{1}^{(5)}\right)^{2}+3\left(\tau_{3}^{(5)}\right)^{2}+4\left(\tau_{5}^{\prime(5)}\right)^{2}+\left(\tau^{(6)}-\tau^{*(4)}\right)^{2}+\left(\tau^{\prime(6)}-\tau^{\prime *(4)}\right)^{2}} .
$$

La minimización se ha realizado con la función extrema de Maple V4. Esta función tiene como parámetros la expresión de la cual se quieren obtener los extremos, las variables y ocasionalmente condiciones frontera. En nuestro caso, las condiciones frontera proceden de una serie de restricciones adicionales que imponemos sobre el tamaño de los coeficientes para evitar el excesivo crecimiento de los errores de redondeo. En concreto imponemos que $\alpha$ tome valores entre 0 y 1 y $\beta$ entre -1 y 1. Así el método óptimo es aquel en el que los parámetros libres toman los valores $\alpha=1047 / 1250$ y $\beta=-1183 / 200000$ y por tanto los coeficientes del método son los de la Tabla 2.5 .

Para estos coeficientes los valores de $A^{(5)}, A^{\prime(5)}$ y $A^{\prime(6)}$ son 0.0023 , 0.00262 y 0.0007 respectivamente. La norma $A^{(6)}$ es despreciable respecto al resto.

El método óptimo obtenido lo denotaremos por $\mathrm{RKN} h^{2} 4: 5 \mathrm{M}$. La letra M significa cerca del mínimo (véase Dormand [19]).

Con el fin de estudiar la eficiencia del método óptimo construido mostraremos los resultados obtenidos al integrar numéricamente tres de los problemas ya considerados en la sección 2.2.3: el problema del oscilador de Duffing para $\varepsilon=0.001$, el problema del satélite artificial ecuatorial con alta excentricidad y la perturbación debida al achatamiento terrestre y el sistema de osciladores. Compararemos el método óptimo RKN $h^{2} 4: 5 \mathrm{M}$ con el esquema de orden oscilatorio 5 de la Tabla 2.1, que denotaremos por RKN $h^{2} 4: 5$, y con el código $\mathrm{RKN} h^{2} 4: 6$, que acabamos de ver presentaba un buen comportamiento. 


\begin{tabular}{cc|ccc} 
& 0 & & \\
$c_{i}$ & $\frac{219}{641}$ & $\frac{47961}{821762}$ & & $a_{i j}$ \\
& $\frac{1047}{1250}$ & $\frac{11132259957}{285156250000}$ & $\frac{88896811293}{285156250000}$ & \\
\hline $\bar{b}_{i}$ & $\frac{143627}{1375758}$ & $\frac{86695891}{261076689}$ & $\frac{79296875}{1248161157}$ \\
$\bar{b}_{i}^{*}$ & $\frac{-657115973}{164250000000}$ & $\frac{1628654723}{164250000000}$ & $\frac{-1183}{200000}$ \\
\hline$b_{i}$ & $\frac{143627}{1375758}$ & $\frac{263374721}{522153378}$ & $\frac{488281250}{1248161157}$ \\
$b_{i}^{*}$ & $\frac{-23375}{2751516}$ & $\frac{14983375}{1044306756}$ & $\frac{-14609375}{2496322314}$
\end{tabular}

Tabla 2.5: Método $\mathrm{RKN} h^{2} 4: 5 \mathrm{M}$ 
Figura 2.6: Oscilador de Duffing. $\varepsilon=10^{-3}$.

En las Figuras 2.6 y 2.7 se presentan los resultados obtenidos al integrar el problema del oscilador de Duffing con $\varepsilon=10^{-3}$ y el problema del satélite con una órbita altamente excéntrica respectivamente. Los tamaños de paso considerados se han variado entre $1 / 2$ y $1 / 2^{7}$ para la integración numérica del primer problema, y entre $1 / 2$ y $1 / 2^{6}$ para el segundo. En ambos casos el método óptimo proporciona mejores resultados que el correspondiente método particular $\mathrm{RKN} h^{2} 4: 5$ y un excelente comportamiento comparado con el método RKN $h^{2} 4: 6$. Cuando la integración se realiza con los tamaños de paso más pequeños las diferencias entre los métodos $\mathrm{RKN} h^{2} 4: 6$ y $\mathrm{RKN} h^{2} 4: 5 \mathrm{M}$ no son significativas. Esto puede ser debido a que para valores de $h$ suficientemente pequeños, los términos que predominan en los errores de truncación local en ambos mtodos son los correspondientes a $h^{5}$.

Por último, en la Figura 2.8 se muestran los resultados de la integración numérica del sistema de osciladores. En este caso se han utilizado tamaños de paso que varían entre $1 / 2$ y $1 / 2^{6}$ para el método RKN $h^{2} 4: 6$ y entre $1 / 2$ y $1 / 2^{8}$ para los otros dos códigos. Como vi- 
mos en la sección 2.2.3, el método particular de orden oscilatorio 5 no proporcionaba tan buenos resultados como el de orden oscilatorio 6. Como se puede ver, el método $\mathrm{RKN} h^{2} 4: 5 \mathrm{M}$ mejora los resultados obtenidos aunque el esquema RKN $h^{2} 4: 6$ sigue siendo el más eficiente.

Figura 2.7: Problema del satélite. $e=0.99$. 
Figura 2.8: Sistema de osciladores. 
2. Nuevos métodos tipo Runge-Kutta-Nyström 


\section{Capítulo 3}

\section{Métodos de paso variable}

\section{$3.1 \quad$ Introducción}

En problemas del tipo (2.2) no excesivamente complejos la aproximación de las soluciones mediante la integración numérica con paso fijo, empleando pasos pequeños con el fin de conseguir gran precisión, conduce a soluciones numéricas suficientemente buenas. Sin embargo, en muchos problemas prácticos las derivadas de la función $f$ varían de forma significativa en magnitud, dando lugar a una gran variación en los errores locales que se producen paso a paso (recordemos que éstos dependen de las diferenciales elementales). Entonces sería más conveniente emplear métodos que permitieran mantener acotados los errores locales adecuando los tamaños de paso a lo largo de la integración. En este contexto surgen los métodos de paso variable, de los cuales los más empleados con esquemas Runge-Kutta y Runge-Kutta-Nyström son los denominados pares encajados (véase Dormand [19], Dormand et al. [20, 21] y Hairer et al. [54]). Una implementación eficiente de un método de paso variable requiere, por tanto, de un mecanismo de control de la amplitud del paso $h$, que será más pequeña en aquellas zonas en las que la solución varíe rápidamente y aumentará en las zonas más sencillas de integrar. Un par encajado $\operatorname{RKN} q(p) s$ consta de dos métodos RKN de $s$ etapas, de órdenes $p$ y $q$, con $p \neq q$, de forma que las etapas intermedias, $k_{i}$, son las mismas para ambos. La integración avanza con el método de orden $q$ mientras que el método de orden $p$ sólo se utiliza para obtener una estimación del error local necesaria, como veremos seguidamente, en el mecanismo de control de paso. Por lo general se consideran pares $\operatorname{RKN} q(p)$ con $p<q$, es decir, 
se aplica el modo conocido como extrapolación local (véase Dormand [19] y Dormand et al. [20, 21]). En este caso se utiliza la aproximación de orden más alto (en general más precisa) para avanzar en la integración, y el método auxiliar de orden $p$ para obtener una estimación del error local. Como veremos a continuación, dicha estimación no se refiere al error local cometido por el método con el que se efectúa la integración, sino al cometido por el método de orden más bajo. Esto resulta ventajoso, porque se pueden minimizar los coeficientes de error del método de orden $q$ (con el fin de obtener un método óptimo) sin que se perjudique con ello la estimación del error. A lo largo de esta sección veremos además qué criterios se han de seguir para que la estimación del error local conseguida con el método de orden $p$ sea lo más fiable posible. Consideremos un par $\operatorname{RKN} q(p) s$ explícito con $p<q$

$$
\begin{aligned}
& \hat{y}_{n+1}=\hat{y}_{n}+h_{n} \hat{\phi}\left(x_{n}, \hat{y}_{n}, \hat{y}_{n}^{\prime}, h_{n}\right), \\
& \hat{y}_{n+1}^{\prime}=\hat{y}_{n}^{\prime}+h_{n} \hat{\phi}^{\prime}\left(x_{n}, \hat{y}_{n}, \hat{y}_{n}^{\prime}, h_{n}\right), \\
& y_{n+1}=\hat{y}_{n}+h_{n} \phi\left(x_{n}, \hat{y}_{n}, \hat{y}_{n}^{\prime}, h_{n}\right), \\
& y_{n+1}^{\prime}=\hat{y}_{n}^{\prime}+h_{n} \phi\left(x_{n}, \hat{y}_{n}, \hat{y}_{n}^{\prime}, h_{n}\right),
\end{aligned}
$$

donde

$$
\begin{aligned}
\hat{\phi}\left(x_{n}, \hat{y}_{n}, \hat{y}_{n}^{\prime}, h_{n}\right) & =\hat{y}_{n}^{\prime}+h_{n} \sum_{i=1}^{s} \hat{\bar{b}}_{i} k_{i}, \\
\hat{\phi}^{\prime}\left(x_{n}, \hat{y}_{n}, \hat{y}_{n}^{\prime}, h_{n}\right) & =\sum_{i=1}^{s} \hat{b}_{i} k_{i}, \\
\phi\left(x_{n}, \hat{y}_{n}, \hat{y}_{n}^{\prime}, h_{n}\right) & =\hat{y}_{n}^{\prime}+h_{n} \sum_{i=1}^{s} \bar{b}_{i} k_{i}, \\
\phi^{\prime}\left(x_{n}, \hat{y}_{n}, \hat{y}_{n}^{\prime}, h_{n}\right) & =\sum_{i=1}^{s} b_{i} k_{i},
\end{aligned}
$$

siendo las $s$ etapas intermedias

$$
k_{i}=f\left(x_{n}+c_{i} h_{n}, \hat{y}_{n}+c_{i} h_{n} \hat{y}_{n}^{\prime}+h_{n}^{2} \sum_{j=1}^{i-1} a_{i j} k_{j}\right),
$$

con $i=1, \ldots, s$ y $c_{1}=0$. En las expresiones anteriores $\hat{y}_{n}$ y $\hat{y}_{n}^{\prime}$ son aproximaciones a la solución y su derivada en el punto $x_{n}$ propor- 
cionadas por las fórmulas de orden $q ; y_{n}$ e $y_{n}^{\prime}$ son las aproximaciones proporcionadas por las fórmulas de orden $p$ y $h_{n}=x_{n+1}-x_{n}$.

Si la magnitud del error local se intenta mantener por debajo de una tolerancia Tol, y el valor $E$ representa la estimación del máximo error local cometido en las aproximaciones a la solución y su derivada, el mecanismo de cambio de paso funciona como sigue

- Si $E \geq$ Tol, la longitud de paso $h_{n}$ resulta demasiado grande y se rechaza la última aproximación, $\hat{y}_{n+1}, \hat{y}_{n+1}^{\prime}$, que se calcula otra vez a partir de la anterior disminuyendo el tamaño de paso. La longitud del paso viene dada por la fórmula

$$
h_{n+1}=\mathrm{Fac} \cdot h_{n} \cdot\left(\frac{\mathrm{Tol}}{E}\right)^{1 /(p+1)},
$$

siendo Fac un factor de seguridad (véase Dormand [19], y Dormand et al. $[20,21])$ que oscila entre 0.75 y 0.95 y que modera los cambios de paso. También se suele introducir un paso máximo $h_{\text {máx }}$ y uno mínimo $h_{\text {mín }}$ de modo que la expresión anterior en la práctica se usa como sigue

$$
h_{n+1}=\operatorname{máx}\left(h_{m i n}, \operatorname{mín}\left(h_{m a ́ x}, \mathrm{Fac} \cdot h_{n} \cdot\left(\frac{\mathrm{Tol}}{E}\right)^{1 /(p+1)}\right)\right) \text {. }
$$

De esta forma se evitan tamaños de paso excesivamente grandes, que darían lugar a errores de desbordamiento, o excesivamente pequeños, que producirían un incremento inacceptable de los errores de redondeo. que llevarían a rechazos posteriores.

- Si $E<$ Tol, la longitud de paso $h_{n}$ es suficientemente pequeña y se acepta el último paso dado. Se avanza un paso, desde $x_{n+1}$ al punto $x_{n+2}=x_{n+1}+h_{n+1}$, con $h_{n+1}$ dado por (3.1).

Denotando por $\delta_{n+1}$ y $\delta_{n+1}^{\prime}$ las diferencias

$$
\begin{aligned}
& \delta_{n+1}=\hat{y}_{n+1}-y_{n+1}, \\
& \delta_{n+1}^{\prime}=\hat{y}_{n+1}^{\prime}-y_{n+1}^{\prime},
\end{aligned}
$$

la estimación $E$ se calcula como

$$
E=\operatorname{máx}\left(\left\|\delta_{n+1}\right\|,\left\|\delta_{n+1}^{\prime}\right\|\right) .
$$


Nota 3.1.1 Se puede elegir cualquier norma, aunque nosotros utilizaremos la norma euclídea

Nota 3.1.2 En cuanto a la estimación del error, se puede estimar el error absoluto, relativo o ponderarlo de manera distinta en cada una de las componentes según el problema considerado. También es posible estimar sólo el error cometido al aproximar la posición $E=\left\|\delta_{n+1}\right\|$ aunque los resultados suelen ser peores (véase Dormand et al. [20, 21]).

Nota 3.1.3 La fórmula de cambio de paso considerada en (3.1) se obtiene tratando de controlar que el error por paso se mantenga por debajo de la tolerancia fijada. Otra alternativa sería tratar de controlar el error por unidad de paso como se sugiere en Lambert [69].

En realidad $\delta_{n+1}$ y $\delta_{n+1}^{\prime}$ son estimaciones de los errores locales cometidos por el método de orden $p$ y no del método de orden $q$ con el que avanzamos en la integración pues

$$
\begin{aligned}
\delta_{n+1} & =\hat{y}_{n+1}-y_{n+1}=\left(y\left(x_{n}+h_{n}\right)-y_{n+1}\right)-\left(y\left(x_{n}+h_{n}\right)-\hat{y}_{n+1}\right) \\
& =e_{n+1}+O\left(h^{q+1}\right)=O\left(h^{p+1}\right) \\
\delta_{n+1}^{\prime} & =\hat{y}_{n+1}^{\prime}-y_{n+1}^{\prime}=\left(y^{\prime}\left(x_{n}+h_{n}\right)-y_{n+1}^{\prime}\right)-\left(y^{\prime}\left(x_{n}+h_{n}\right)-\hat{y}_{n+1}^{\prime}\right) \\
& =e_{n+1}^{\prime}+O\left(h^{q+1}\right)=O\left(h^{p+1}\right)
\end{aligned}
$$

donde $e_{n+1}$ y $e_{n+1}^{\prime}$ representan los errores locales para el método de orden $p$. Para un buen funcionamiento del código es deseable que las estimaciones del error del método de orden bajo sean mayores que la del método de orden alto. Teniendo en cuenta que los errores de truncación local para un método de orden $m$ para las aproximaciones a la solución y su derivada se pueden escribir (véase Dormand [19]) respectivamente

$$
\sum_{i=m+1}^{\infty} h^{i} \sum_{j=1}^{n_{i}} \tau_{j}^{(i)} F_{j}^{(i)}, \quad \sum_{i=m+1}^{\infty} h^{i} \sum_{j=1}^{n_{i}} \tau_{j}^{(i)} F_{j}^{(i+1)},
$$

es claro que

$$
\begin{aligned}
& \delta_{n+1}=\sum_{i=p+1}^{\infty} h^{i} \sum_{j=1}^{n_{i}}\left(\tau_{j}^{(i)}-\hat{\tau}_{j}^{(i)}\right) F_{j}^{(i)} \\
& \delta_{n+1}^{\prime}=\sum_{i=p+1}^{\infty} h^{i} \sum_{j=1}^{n_{i}}\left(\tau_{j}^{\prime(i)}-\hat{\tau}_{j}{ }^{(i)}\right) F_{j}^{(i+1)} .
\end{aligned}
$$


Una vez obtenido el par encajado $\operatorname{RKN} q(p)$ conviene establecer una serie de criterios para elegir los parámetros libres de forma que se obtenga un buen par encajado (véase Dormand et al. [20]). Nos interesa por un lado que el método de orden $q$ sea óptimo y por otro que la estimación del error sea lo más fiel posible. Esto se traduce en lo siguiente:

1. Puesto que la integración avanza con la fórmula de orden $q \mathrm{y}$ el correspondiente error local se comporta como $O\left(h^{q+1}\right)$ parece lógico intentar minimizar los coeficientes que acompañan a $h^{q+1}$. Considerando que todas las diferenciales elementales tienen la misma contribución al error lo que se trata de hacer es

$$
A^{(q+1)}=\left\|\tau^{(q+1)}\right\|, \quad A^{\prime(q+1)}=\left\|\tau^{\prime(q+1)}\right\|,
$$

tan pequeños como sea posible.

2. Puesto que la fórmula (3.1) se basa en que el estimativo sea proporcional a $h^{p+1}$, habrá que pedir que en las estimaciones del error, tanto para la solución como para la derivada, dominen los términos en $h^{p+1}$. Teniendo en cuenta (3.2) y (3.3) bastará con pedir que

$$
C^{(p+1)}=\frac{\left\|\tau^{(p+2)}-\hat{\tau}^{(p+2)}\right\|}{\left\|\tau^{(p+1)}\right\|}, \quad C^{(p+1)}=\frac{\left\|\tau^{\prime(p+2)}-\hat{\tau}^{\prime(p+2)}\right\|}{\left\|\tau^{\prime(p+1)}\right\|},
$$

sean lo más pequeños posibles.

3. Se ha de pedir además que en el método de orden $p$ dominen los términos en $h^{p+1}$ frente a aquellos en $h^{p+2}$. Esto se consigue minimizando

$$
B^{(p+2)}=\frac{\left\|\tau^{(p+2)}\right\|}{\left\|\tau^{(p+1)}\right\|}, \quad B^{\prime(p+2)}=\frac{\left\|\tau^{\prime(p+2)}\right\|}{\left\|\tau^{\prime(p+1)}\right\|} .
$$

De esta forma conseguimos que las dos fórmulas sean suficientemente distintas, ya que es muy habitual elegir $q=p+1$.

4. Por último como recomendación general, hay que tomar valores para los coeficientes no demasiado grandes para evitar los posibles errores de redondeo. 
En las siguientes secciones trataremos de extender las ideas expuestas para métodos RKN a los métodos de tipo $\mathrm{RKN} h^{2}$ que hemos introducido en el capítulo anterior. Siguiendo la notación introducida denotaremos estos pares por RKN $h^{2} q: q^{\prime}\left(p: p^{\prime}\right)$ siendo $q$ y $p$ el orden por las fórmulas de orden mayor y menor respectivamente conseguido para un problema general y $q^{\prime}$ y $p^{\prime}$ los órdenes oscilatorios correspondientes.

\subsection{Construcción de un par encajado RKN $h^{2} 4: 6(3: 4)$}

Utilizando las ideas de la sección anterior, construiremos un par que constará de dos fórmulas $\mathrm{RKN} h^{2}$ : una de ellas de orden 4 y orden oscilatorio 6 que será la que usaremos para hacer avanzar la integración y otra fórmula de orden $3 \mathrm{y}$ orden oscilatorio 4 que emplearemos para estimar el error. El método de orden 4 es el obtenido en la sección 2.2.2 cuyos coeficientes son los de la Tabla 2.2, que como vimos es el único método de tipo $\mathrm{RKN}^{2}$ con orden oscilatorio 6 . Teniendo en cuenta las condiciones de orden de la sección 2.3.3, para obtener la fórmula de orden 3 con orden oscilatorio 4 será necesario resolver el siguiente sistema de ecuaciones:

$$
\begin{aligned}
\bar{b}_{1}+\bar{b}_{2}+\bar{b}_{3} & =1 / 2, \\
\bar{b}_{2} c_{2}+\bar{b}_{3} c_{3} & =1 / 6, \\
\bar{b}_{1}^{*}+\bar{b}_{2}^{*}+\bar{b}_{3}^{*} & =\left(\bar{b}_{2} c_{2}^{2}+\bar{b}_{3} c_{3}^{2}-1 / 12\right) / 2, \\
b_{1}+b_{2}+b_{3} & =1, \\
b_{2} c_{2}+b_{3} c_{3} & =1 / 2, \\
b_{2} c_{2}^{2}+b_{3} c_{3}^{2} & =1 / 3, \\
b_{1}^{*}+b_{2}^{*}+b_{3}^{*} & =0, \\
b_{2}^{*} c_{2}+b_{3}^{*} c_{3} & =b_{3} a_{32} c_{2}-1 / 24 .
\end{aligned}
$$

Consideraremos las condiciones de suma de fila (2.17) y (2.18). Siguiendo la filosofía de los pares $\operatorname{RKN} q(p)$ de la sección precedente, los coeficientes $a_{i j}$ y $c_{i}$ coinciden con los de la fórmula de orden alto. Resolviendo el sistema anterior resulta una familia de métodos con cuatro parámetros libres. De forma análoga a los pares $\operatorname{RKN} q(p)$ trataremos ahora de que estos parámetros hagan mínimos los correspondientes 
$C^{(4)}, C^{\prime(4)}, B^{(5)}$ y $B^{\prime(5)}$ buscando que el estimativo sea proporcional a $h^{4}$ y que en la fórmula de orden menor dominen los términos en $h^{4}$ frente a los términos en $h^{5}$. Para los métodos considerados las constantes son las que siguen:

$$
\begin{aligned}
C^{(4)} & =\frac{\left\|\tau^{(5)}+\tau^{*(3)}-\left(\hat{\tau}^{(5)}+\hat{\tau}^{*(3)}\right)\right\|}{\left\|\tau^{(4)}+\tau^{*(2)}\right\|}, \\
C^{\prime(4)} & =\frac{\left\|\tau^{\prime(5)}+\tau^{\prime *(3)}-\left(\hat{\tau}^{\prime(5)}+\hat{\tau}^{\prime *(3)}\right)\right\|}{\left\|\tau^{\prime(4)}+\tau^{\prime *(2)}\right\|}, \\
B^{(5)} & =\frac{\left\|\tau^{(5)}+\tau^{*(3)}\right\|}{\left\|\tau^{(4)}+\tau^{*(2)}\right\|}, \\
B^{\prime(5)} & =\frac{\left\|\tau^{\prime(5)}+\tau^{\prime *(3)}\right\|}{\left\|\tau^{\prime(4)}+\tau^{\prime *(2)}\right\|} .
\end{aligned}
$$

Buscamos que los grados de libertad minimicen las funciones

$$
\begin{aligned}
& \Phi=\sqrt{C^{(4)^{2}}+B^{(5)^{2}}}, \\
& \varphi=\sqrt{C^{\prime}(4)^{2}+B^{\prime(5)^{2}}} .
\end{aligned}
$$

Empleando una vez más la función extrema de Maple V 4, los coeficientes del método son los de la Tabla 3.1. Para estos coeficientes

$$
C^{(4)}=0.65, \quad B^{(5)}=0.14, \quad C^{\prime(4)}=0.077, \quad B^{\prime(5)}=0.178 .
$$

Si se considera como referencia el método RKN4(3) propuesto en Dormand et al. [20] para el cual los valores de las constantes son

$$
C^{(4)}=1.02, \quad B^{(5)}=1.03, \quad C^{\prime(4)}=1.19, \quad B^{\prime(5)}=1.20,
$$

vemos que las minimizaciones en nuestro caso han sido hechas de forma eficiente.

\subsection{Experimentos numéricos}

Con el fin de ilustrar el buen comportamiento del par encajado construido mostraremos los resultados obtenidos al integrar numéricamente tres problemas oscilatorios: 


\begin{tabular}{cc|ccc} 
& 0 & & & $a_{i j}$ \\
$c_{i}$ & $\frac{2}{9}$ & $\frac{2}{81}$ & & \\
& $\frac{19}{24}$ & $\frac{-1235}{18432}$ & $\frac{779}{2048}$ & \\
\hline & $\bar{b}_{i}$ & $\frac{-296317}{19416860}$ & $\frac{17750961}{41899540}$ & $\frac{18231592}{199022815}$ \\
& $\bar{b}_{i}^{*}$ & $\frac{-386269}{117727488}$ & $\frac{1}{1280}$ & 0 \\
\hline & $b_{i}$ & $\frac{1}{76}$ & $\frac{81}{164}$ & $\frac{384}{779}$ \\
$b_{i}^{*}$ & $\frac{-2}{95}$ & $\frac{6}{205}$ & $\frac{-32}{3895}$
\end{tabular}

Tabla 3.1: Método de orden bajo para el par $\mathrm{RKN} h^{2} 4: 6(3: 4)$ 
1. El oscilador de Duffing (2.68) con $\varepsilon=10^{-3}$, que es un problema que hemos venido considerando en los experimentos anteriores. Éste no presenta serios problemas en su integración que den lugar a muchos rechazos y a la consecuente reducción de la amplitud de paso. Por ello el paso mínimo permitido a lo largo de la integración ha sido $10^{-3}$.

2. El problema del satélite artificial ya considerado (2.69), integrando una órbita ecuatorial con alta excentricidad y teniendo en cuenta, como anteriormente, sólo la perturbación debida al armónico zonal $J_{2}$. Este problema formulado en variables BF (Burdet-Ferrándiz) presenta gran regularidad ya que las derivadas no varían rápidamente. El paso mínimo utilizado coincide con el del problema anterior.

3. El problema de Bessel, que tiene por ecuación:

$$
y^{\prime \prime}+100 y=-\frac{y}{4 x^{2}},
$$

cuyos valores iniciales han sido tomados de la solución casi periódica $(y(x) \approx y(x+2 \pi / 10))$

$$
\sqrt{x} J_{0}(10 x)
$$

siendo $J_{0}$ la función de Bessel de primera especie. Este problema ha sido utilizado por varios autores con diversos propósitos como podemos ver en Gautschi [41], van der Houwen y Sommeijer [61] y Neta y Ford [90]. Nosotros lo utilizaremos como test de eficiencia para los pares encajados, pues a medida que el extremo inferior de integración se aproxima a cero aumenta la dificultad de integración, ya que en $x=0$ la función no es derivable y cerca de él los valores de la derivada difieren enormemente. En este sentido hemos considerado tres intervalos de integración $[1,10],[0.1,10]$ y $[0.01,10]$ para poner de manifiesto el comportamiento de los diferentes métodos a medida que el problema se hace más difícil de integrar. El paso mínimo permitido en todos los algoritmos ha sido de $10^{-4}$ en la integración en los dos primeros intervalos, salvo en el método RKGM4(3) para el que se ha considerado un valor de $10^{-5}$. En el último intervalo se ha 
realizado la integración con un tamaño de paso mínimo de $10^{-5}$ en todos los esquemas.

Los métodos utilizados en la integración numérica de los anteriores problemas han sido los que siguen:

- Los algoritmos desarrollados en el presente capítulo implementados tanto en paso fijo como en paso variable. En las gráficas nos referiremos a ellos como $\mathrm{RKN} h^{2} 4: 6$ para el método de paso fijo y como RKN $h^{2} 4: 6(3: 4)$ para el par encajado. En ambos casos se requieren tres evaluaciones de función por paso.

- El par encajado RKN4(3) con cuatro etapas desarrollado por Dormand et al. [20]. Dicho método tiene propiedad FSAL por lo que, salvo en el primero, el método avanza realizando sólo tres evaluaciones de función por paso.

- El par encajado diseñado especialmente para problemas oscilatorios y debido a González et al. [44, 47, 48] que requiere cuatro evaluaciones de función por paso. Lo denotaremos por RKGM4(3) en las gráficas.

Todas las integraciones han sido realizadas considerando un paso inicial $h=0.1$. Cuando la elección del tamaño de paso inicial resulte crítica, existen alternativas para seleccionar dicho tamaño de paso de forma adecuada al problema que se desee integrar (veáse Gladwell et al. [43] y Hairer et al. [54]), aunque en los problemas citados no ha sido necesario.

Las gráficas presentadas son gráficas de eficiencia en las que se representa el logaritmo decimal del error a lo largo de la integración frente al logaritmo decimal del número de evaluaciones necesarias. En algún caso incluiremos además gráficas de número de evaluaciones de función frente a tiempo de CPU empleado, que en el caso de integraciones con métodos de paso variable también es una buena forma de ilustrar la eficiencia de los métodos. Los cálculos han sido realizados con precisión aritmética de 18 dígitos.

La Figura 3.1 muestra la integración del oscilador de Duffing con $\varepsilon=10^{-3}$ al cabo de 10 revoluciones. Se han tomado tolerancias que varían de $10^{-3}$ a $10^{-7}$ para el método RKN4(3), de $10^{-4}$ a $10^{-14}$ para el esquema RKGM4(3) y de $10^{-3}$ a $10^{-13}$ para el código RKN $h^{2} 4: 6(3: 4)$. 
Figura 3.1: Oscilador de Duffing. $\varepsilon=10^{-3}$.

Para el algoritmo $\mathrm{RKN}^{2} 4: 6$ se han empleado tamaños de paso entre $1 / 2$ y $1 / 2^{8}$. La gráficas ponen de manifiesto que el par encajado RKN $h^{2} 4: 6(3: 4)$ resulta ser mucho más eficiente que los métodos RKGM4(3) y RKN4(3). Las diferencias son aún mayores si representamos el tiempo de CPU empleado en realizar las integraciones. Como vemos en la Figura 3.2, el esquema $\mathrm{RKN}^{2} 4: 6(3: 4)$ requiere un coste computacional en tiempo de CPU mucho menor que los otros dos algoritmos, especialmente que el método RKGM4(3). Este último, además de emplear una evaluación de función más por paso, tiene unos coeficientes que dependen de $h$ de forma no tan sencilla como el método que hemos desarrollado y recalcularlos cada vez que el tamaño de paso varía, supone un coste computacional nada despreciable para problemas con funciones no excesivamente caras de evaluar. Volviendo sobre la Figura 3.1, si comparamos los métodos RKNh $h^{2} 4: 6$ y RKN $h^{2} 4: 6(3: 4)$ observamos que el esquema implementado en paso fijo presenta unos resultados ligeramente más precisos que el par encajado. Esto puede ser debido, como ya apuntábamos anteriormente, al hecho de que este 
Figura 3.2: Oscilador de Duffing. $\varepsilon=10^{-3}$.

problema no presenta dificultades de integración y por ello un buen método implementado en paso fijo debería ser suficiente para obtener buenas aproximaciones.

En la Figura 3.3 observamos los resultados de integrar el problema del satélite artificial con $e=0.99$. En este problema las tolerancias empleadas varían de $10^{-3}$ a $10^{-8}$ para el método RKN4(3), de $10^{-3}$ a $10^{-14}$ para el esquema $\operatorname{RKGM} 4(3)$ y de $10^{-3}$ a $10^{-12}$ para el algoritmo $\mathrm{RKN}^{2}$ 4:6(3:4). Para el esquema en paso fijo hemos utilizado tamaños de paso que oscilan entre $1 / 2$ y $1 / 2^{7}$. En este caso el método $\mathrm{RKN} h^{2} 4: 6(3: 4)$ presenta los mejores resultados, proporcionando soluciones más precisas que los otros dos pares encajados. Cuando no se requiera mucha precisión, el método implementado en paso fijo emplea menos evaluaciones de función que el correspondiente método de paso variable. Sin embargo, éste último proporciona resultados más aproximados cuando se requiera una precisión mayor, aunque la diferencia sigue siendo mínima. De nuevo nos enfrentamos a un problema de no excesiva dificultad para ser integrado en paso fijo. La variación en 
Figura 3.3: Problema del satélite. $e=0.99$.

el tamaño de paso no es importante, de modo que los dos esquemas se comportan de modo muy similar. Como vemos en la Figura 3.4 la ventaja de nuestros métodos frente a los esquemas RKGM4(3) y RKN4(3) en tiempo de CPU es clara.

En las Figuras 3.5, 3.6 y 3.8 se tienen los resultados de integrar el problema de Bessel en los intervalos $[1,10],[0.1,10]$ y $[0.01,10]$. Las tolerancias para la integración en el primer intervalo varían de

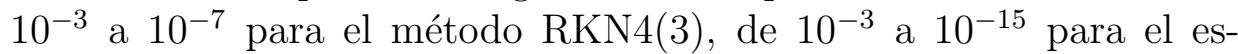
quema RKGM4(3) y de $10^{-3}$ a $10^{-12}$ para el método $\mathrm{RKN} h^{2} 4: 6(3: 4)$. En el segundo intervalo las tolerancias empleadas varían hasta $10^{-14}$ para el método RKGM4(3); para el resto de códigos las tolerancias utilizados coinciden con los del intervalo $[1,10]$. En el último intervalo se han utilizado valores de tolerancias entre $10^{-3}$ y $10^{-7}$ para el método RKN4(3), y entre $10^{-3}$ y $10^{-14}$ para los esquemas RKGM4(3) y RKN $h^{2} 4: 6(3: 4)$. En los tres casos, se han empleado tamaños de paso que oscilan entre $1 / 2^{5}$ y y $1 / 2^{11}$ para el código $\mathrm{RKN} h^{2} 4: 6$. 
Figura 3.4: Problema del satélite. $e=0.99$.

Como se puede apreciar en la Figura 3.5, en el intervalo $[1,10]$ los métodos RKNh $h^{2}$ 4:6(3:4) se muestran menos eficientes que el esquema RKGM4(3) cuando se requieran soluciones no demasiado precisas. Sin embargo, para errores menores los métodos RKN $h^{2} 4: 6(3: 4)$ y RKN $h^{2} 4: 6$ muestran un comportamiento muy similar, proporcionando los mejores resultados. En tiempo de CPU nuestros método aventajarán aún más, como hemos visto en los problemas anteriores, al método RKGM4(3). En la Figura 3.6 se pone de manifiesto que en el intervalo $[0.1,10]$ el método RKGM4(3) presenta unos resultados ligeramente mejores que los esquemas $\mathrm{RKN} h^{2} 4: 6(3: 4)$ y $\mathrm{RKN} h^{2} 4: 6$. No obstante, como se puede ver en la Figura 3.7, el método RKGM4(3) requiere un coste en tiempo de CPU mucho mayor. Además, este algoritmo requiere tamaños de paso menores para obtener resultados comparables.

En estos dos intervalos, podemos observar que el comportamiento del método de paso fijo es muy similar al del correspondiente método de paso variable cuando se requieran los soluciones más precisas. Esto 
Figura 3.5: Problema de Bessel. $x \in[1,10]$

puede ser debido a que el número de rechazos en la integración en paso variable es pequeña y por ello el comportamiento está próximo al de los métodos de paso fijo. Sin embargo, para obtener estos resultados, ha sido necesario integrar el problema en paso fijo con pasos menores que en los problemas anteriores. Por el contrario, como muestra la Figura 3.8 correspondiente a la integración del problema de Bessel en $[0.01,10]$, cuando el extremo inferior del intervalo está más próximo a cero, el método de paso fijo deja de tener un comportamiento comparable con los algoritmos de paso variable RKN4(3), RKN $h^{2} 4: 6(3: 4)$ y, especialmente, RKGM4(3). Éste último es el que proporciona los mejores resultados. No obstante, el par RKN $h^{2} 4: 6(3: 4)$ conserva la ventaja frente al par RKGM4(3) de que el coste en tiempo de CPU es bastante menor (Figura 3.9). 
Figura 3.6: Problema de Bessel. $x \in[0.1,10]$ 
Figura 3.7: Problema de Bessel. $x \in[0.1,10]$ 
3. Métodos de paso variable

Figura 3.8: Problema de Bessel. $x \in[0.01,10]$ 
Figura 3.9: Problema de Bessel. $x \in[0.01,10]$ 


\section{Capítulo 4}

\section{Métodos RKN $h^{2}$ de orden alto}

\subsection{Introducción}

En multitud de situaciones es necesario obtener la aproximación a la solución del problema con gran precisión. En estos casos el uso de métodos de orden no demasiado alto, como los expuestos en los capítulos precedentes, pueden llevar al uso de amplitudes de paso excesivamente pequeñas que provocan un gasto computacional considerable. Además, al reducir el paso excesivamente los errores de redondeo dominan a los de truncación local, por lo que hay ciertas barreras a la precisión que se puede alcanzar con un método de orden bajo (véase Dormand [19]). Solamente en aquellos casos en los que se requiera un moderado error puede utilizarse una fórmula de orden bajo. Si no, es preferible el uso de esquemas de orden alto. Más aún, como ya justificábamos en la sección 3.1, la integración numérica de muchos problemas hace necesaria la implementación de los métodos en paso variable. La obtención de los métodos de paso fijo $\mathrm{RKN} h^{2} p: q$ aunque teóricamente clara, nos lleva a tratar con fórmulas y cálculos cada vez más complicados. A medida que aumentamos el orden de los métodos resulta sumamente ventajoso el uso de representaciones gráficas que nos permitan obtener de forma sistemática las ecuaciones de orden y las correspondientes constantes de error. Con este fin, hemos tomado como referencia la teoría de árboles con raíz desarrollada tanto para métodos Runge-Kutta, como para Runge-Kutta-Nyström y de la que podemos encontrar un profundo análisis en Butcher [6], Hairer et al. [54] y Hairer y Wanner [55]. Esta teoría, modificada convenientemente para los métodos que nos ocupan, nos permitirá obtener las ecuaciones 
y constantes de error para conseguir un par encajado $\mathrm{RKN} h^{2}$ de orden elevado.

\subsection{Teoría de árboles para los métodos RKN $h^{2}$}

\subsection{1 Árboles especiales de Nyström}

Antes de comenzar con esta teoría trataremos de motivar la construcción de estos árboles con raíz. Partiendo del problema (4.3), considerábamos los métodos RKN $h^{2}$ de orden $p$ y tratábamos de aproximar las derivadas de la función $f$ mediante evaluaciones de función de la forma siguiente:

$$
\begin{aligned}
& \sum_{j=0}^{p-2} \frac{h^{j} f_{0}^{(j)}}{(j+2) !} \approx \sum_{i=1}^{s}\left(\bar{b}_{i}+h^{2} \omega^{2} \bar{b}_{i}^{*}\right) k_{i}, \\
& \sum_{j=0}^{p-1} \frac{h^{j} f_{0}^{(j)}}{(j+1) !} \approx \sum_{i=1}^{s}\left(b_{i}+h^{2} \omega^{2} b_{i}^{*}\right) k_{i},
\end{aligned}
$$

en las expresiones de la solución y la derivada respectivamente. Desarrollando en serie de Taylor la segunda parte de las expresiones anteriores e identificando términos semejantes, llegábamos a las ecuaciones de orden $p$ que habrían de ser resueltas. Debido a los coeficientes adicionales que presentan los métodos que venimos desarrollando frente a los códigos RKN clásicos, para el problema particular $f=-\omega^{2} y$ podíamos incrementar el orden del método hasta $q$, obteniéndose así algoritmos que integran el problema del oscilador perturbado (2.1) de forma más eficiente que los algoritmos clásicos. De lo que se trata ahora es de evitar el proceso anterior para llegar a tales ecuaciones y, por tanto, de dar una manera sistemática de obtener tanto las derivadas de la función $f$ como de los valores $k_{i}$. Esto lo haremos utilizando la ya mencionada teoría de árboles especiales de Nyström con raíz. Dichos árboles facilitarán así mismo la obtención de expresiones para el error de truncación local y las constantes de error. Una vez hecho esto para un problema general, concretaremos para el caso particular del oscilador no perturbado con el fin de obtener métodos con orden oscilatorio $q$. 
Comencemos recordando los árboles de Nyström con raíz. Para ello consideremos el sistema autónomo de ecuaciones diferenciales

$$
\left(y^{J}\right)^{\prime \prime}=f^{J}\left(y^{1}, \ldots, y^{n}\right),
$$

donde el superíndice $J$ denota la $J$-ésima componente del correspondiente vector. La variable $x$ se puede ajustar al sistema como $x^{\prime \prime}=0$, por ello no supone restricción de generalidad suponer (4.3) independiente de $x$.

Definición 4.2.1 Un árbol especial de Nyström $t$ con raíz de orden $q$ es un grafo con $q$ nodos de dos tipos, gordos y delgados, unidos entre sí. El del nivel anterior a uno fijo se llama padre de éste y él es su hijo. Además se verifican las siguientes condiciones:

- La raíz es siempre gorda.

- Un nodo delgado puede tener a lo sumo un hijo que debe ser gordo.

- Los nodos gordos sólo pueden tener hijos delgados.

Asociadas a todo árbol especial de Nyström tenemos las siguientes funciones

1. $\rho(t)$, conocida como orden de $t$, es el número de nodos.

2. $\alpha(t)$ es el número de etiquetados distintos para un mismo árbol.

3. $\gamma(t)$, conocida como función densidad, es el producto de $\rho(t)$ y todos los órdenes de los árboles que aparecen si las raíces, una detrás de otra, se van quitando de $t$.

4. $F^{J}(t)(y)$, también llamada diferencial elemental, es la suma de un producto de expresiones de la forma

$$
\frac{\partial^{r} f^{J}}{\partial y^{K} \ldots}(y), \quad y^{\prime K}
$$

En lo que sigue emplearemos para las derivadas parciales de $f^{J}$ la notación $f_{K L \ldots}^{J}$. Un factor del tipo $f_{K}^{J} \cdot f^{K}$ aparece si el nodo 
gordo $j$ está conectado vía uno delgado con su hijo $k$. Un factor del tipo $f_{K}^{J} \cdot y^{\prime K}$ aparece si $k$ es el índice de un nodo final delgado. Por tanto, la derivación con respecto a $x$ consiste en añadir una rama con un nodo delgado en cada nodo gordo y una rama con un nodo gordo en cada nodo delgado final. De este modo se van obteniendo sucesivamente los distintos árboles de orden $q$ a partir de los de orden $q-1$. Las etiquetas indican el orden de generación de los nodos siguiendo este procedimiento.

5. $\Phi_{j}(t)$, llamado también peso elemental, es la suma extendida a todos los nodos gordos salvo la raíz $j$ y con un término general que es un producto con factores que pueden ser de dos tipos: $a_{i j}$ si el nodo gordo $i$ tiene un nieto grueso $j$, y $c_{i}^{k}$ si el nodo gordo $i$ tiene $k$ hijos delgados finales.

Representaremos por $S N T_{q}$ (Special Nyström Trees) al conjunto de árboles especiales de Nyström con raíz de orden $q$ y por $L \mathrm{SNT}_{q}$ (Labelled Special Nyström Trees) al conjunto de árboles especiales con raíz etiquetados de orden $q$.

En la Figura 4.1 hemos representado todos $\operatorname{los} S N T_{q}$ con $q$ menor o igual que 5 y las correspondientes funciones asociadas.

\subsubsection{Derivadas de la función $f$}

Derivando sucesivamente respecto a $x$ cada componente $f^{J}$, vamos obteniendo

$$
\begin{aligned}
\left(f^{J}\right)^{(1)} & =\sum_{K} f_{K}^{J} y_{0}^{\prime K}, \\
\left(f^{J}\right)^{(2)} & =\sum_{K, L} f_{K L}^{J} y_{0}^{\prime K} y_{0}^{\prime L}+\sum_{K} f_{K}^{J} f_{0}^{K}, \\
\left(f^{J}\right)^{(3)} & =\sum_{K, L, M} f_{K L M}^{J} y_{0}^{\prime K} y_{0}^{\prime L} y_{0}^{\prime M}+3 \sum_{K, L} f_{K L}^{J} f^{K} y_{0}^{\prime L}+\sum_{K, L} f_{K}^{J} f_{L}^{K} y_{0}^{\prime L},
\end{aligned}
$$

Teorema 4.2.2 Se satisface la siguiente relación

$$
\left(f^{J}\right)^{(q)}=\sum_{t \in S N T_{q+1}} \alpha(t) F^{J}(t)(y)=\sum_{t \in L S N T_{q+1}} F^{J}(t)(y) .
$$




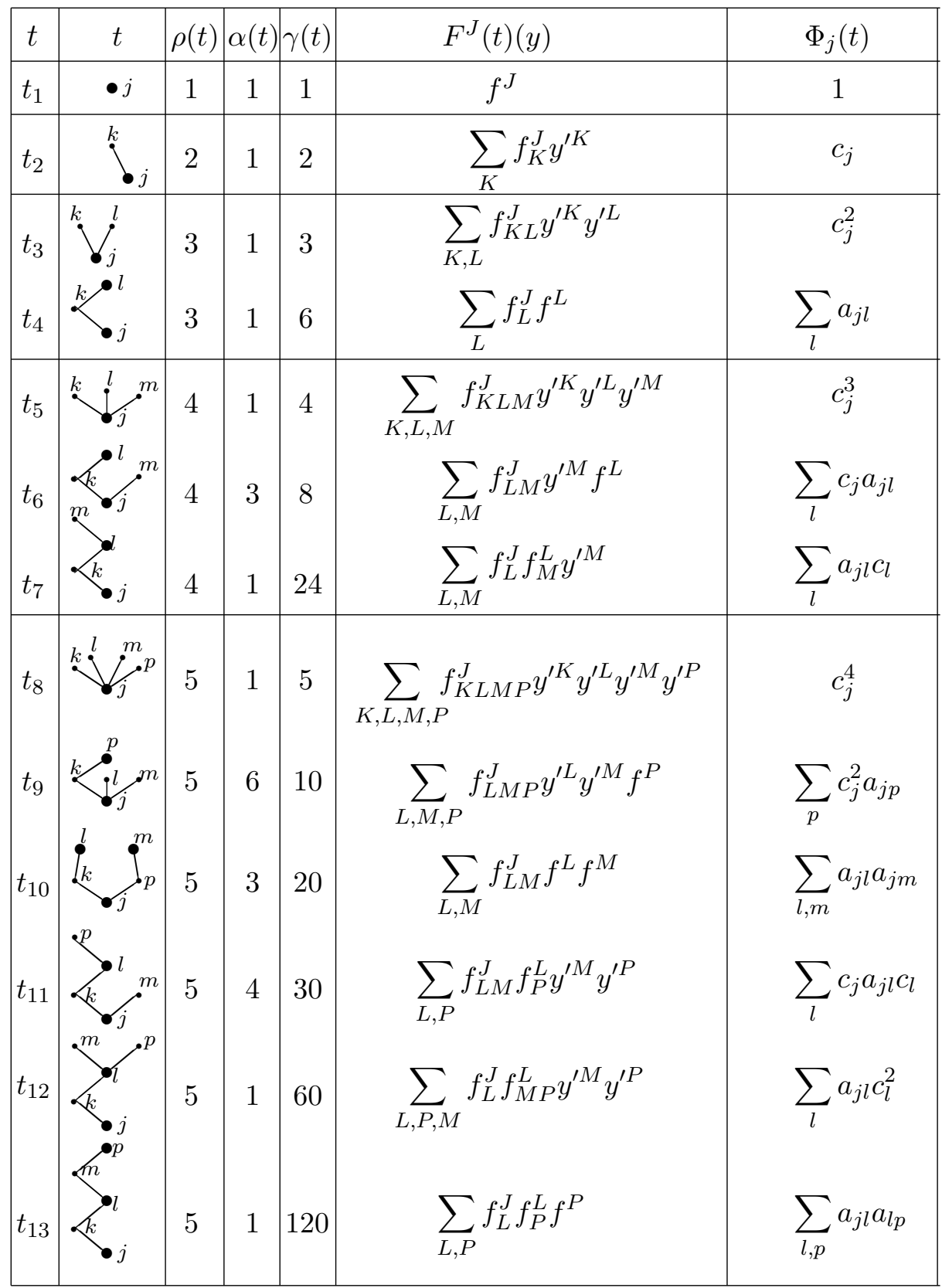

Figura 4.1: $S N T$ hasta orden 5 . 


\section{Demostración:}

Para $q=1,2,3$ basta comparar las derivadas obtenidas anteriormente con los correspondientes árboles de órdenes 2, 3 y 4 de la Figura 4.1. Para valores $q \geq 4$ bastará con seguir un sencillo razonamiento inductivo como el que se puede ver en Hairer et al. [54]. Para obtener la derivada de orden $q$ se han de derivar los correspondientes árboles de dicho orden y geométricamente implica añadir una nueva rama con una nueva letra en cada nodo del modo comentado anteriormente. Siguiendo este proceso es claro que todos los $L S N T$ de orden $q+1$ aparecen en la derivada $q$-ésima una sola vez. Agrupando todos los términos con idénticas diferenciales elementales obtenemos finalmente la expresión de la derivada $q$-ésima. Esto equivale a que los $S N T$ de orden $q+1$ aparezcan multiplicados por $\alpha(t)$.

El teorema anterior nos proporciona una regla general para obtener derivadas de cualquier orden para la función $f$.

\subsubsection{Derivadas de los valores $k_{i}$}

Comenzaremos por reescribir un método $\mathrm{RKN} h^{2}$ de la forma siguiente:

$$
\begin{aligned}
g_{1}^{J} & =y_{0}^{J}, \\
g_{i}^{J} & =y_{0}^{J}+c_{i} h y_{0}^{\prime J}+h^{2} \sum_{j} a_{i j} f^{J}\left(g_{j}\right), \quad i>1, \\
k_{i}^{J} & =f^{J}\left(g_{i}\right), \\
y_{1}^{J} & =y_{0}^{J}+h y_{0}^{\prime J}+\sum_{i=1}^{s}\left(\bar{b}_{i}+h^{2}\left(\omega^{J}\right)^{2} \bar{b}_{i}^{*}\right) k_{i}^{J}, \\
y_{1}^{\prime J} & =y_{0}^{\prime J}+h \sum_{i=1}^{s}\left(b_{i}+h^{2}\left(\omega^{J}\right)^{2} b_{i}^{*}\right) k_{i}^{J} .
\end{aligned}
$$

A la hora de calcular las derivadas de $k_{i}$ es necesario tener en cuenta que para cualquier función $\Phi(h)$ se verifican las relaciones

$$
\begin{aligned}
\left.(h \Phi(h))^{(q)}\right|_{h=0} & =\left.q(\Phi(h))^{(q-1)}\right|_{h=0}, \\
\left.\left(h^{2} \Phi(h)\right)^{(q)}\right|_{h=0} & =\left.q(q-1)(\Phi(h))^{(q-2)}\right|_{h=0},
\end{aligned}
$$


que se prueban fácilmente sin más que aplicar la fórmula de Leibniz. Con estas relaciones es sencillo comprobar que

$$
\begin{aligned}
& \left.\left(g_{i}^{J}\right)^{(1)}\right|_{h=0}=c_{i} y_{0}^{\prime J}, \\
& \left.\left(k_{i}^{J}\right)^{(1)}\right|_{h=0}=\left.\sum_{K} f_{K}^{J}\left(g_{i}\right)\left(g_{i}^{K}\right)^{(1)}\right|_{h=0}=c_{i} \sum_{K} f_{K}^{J} y_{0}^{\prime K}, \\
& \left.\left(g_{i}^{J}\right)^{(2)}\right|_{h=0}=2 \sum_{j} a_{i j} f^{J}, \\
& \left.\left(k_{i}^{J}\right)^{(2)}\right|_{h=0}=c_{i}^{2} \sum_{K, L} f_{K L}^{J} y_{0}^{\prime K} y_{0}^{\prime L}+2 \sum_{J, K} a_{i j} f_{K}^{J} f^{K}, \\
& \left.\left(g_{i}^{J}\right)^{(3)}\right|_{h=0}=6 \sum_{j, K} a_{i j} c_{j} f_{K}^{J} y_{0}^{\prime K} \\
& \left.\left(k_{i}^{J}\right)^{(3)}\right|_{h=0}=c_{i}^{3} \sum_{K, L, M} f_{K L M}^{J} y_{0}^{\prime K} y_{0}^{\prime L} y_{0}^{\prime M}+6 c_{i} \sum_{j, K, L} a_{i j} f_{K L}^{J} f^{K} y_{0}^{\prime L} \\
& +6 \sum_{j, K, L} a_{i j} c_{j} f_{K}^{J} f_{L}^{K} y_{0}^{L L}, \\
& \left.\left(g_{i}^{J}\right)^{(4)}\right|_{h=0}=12 \sum_{j, K, L} a_{i j} c_{j}^{2} f_{K L}^{J} y_{0}^{\prime K} y_{0}^{\prime L}+24 \sum_{j, l, K} a_{i j} a_{j l} c_{j}^{2} f_{K}^{J} f^{K}, \\
& \left.\left(k_{i}^{J}\right)^{(4)}\right|_{h=0}=c_{i}^{4} \sum_{K, L, M, N} f_{K L M N}^{J} y_{0}^{\prime K} y_{0}^{\prime L} y_{0}^{\prime N} y_{0}^{\prime M} \\
& +12 c_{i, K, L, M}^{2} \sum_{i j} f_{K L M}^{J} f^{K} y_{0}^{\prime L} y_{0}^{\prime M} \\
& +24 c_{i, K, L, M} a_{i j} c_{j} f_{K L}^{J} f_{M}^{K} y_{0}^{\prime M} y_{0}^{\prime L}
\end{aligned}
$$

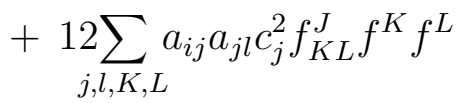

$$
\begin{aligned}
& +12 \sum_{j, K, L, M} a_{i j} c_{j}^{2} f_{K}^{J} f_{K}^{J} f_{L M}^{K} y_{0}^{\prime L} y_{0}^{\prime M}
\end{aligned}
$$

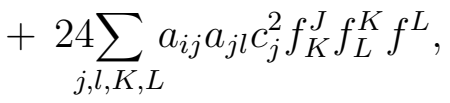

Aunque lo hemos omitido por abreviar la notación, se entiende que cada $f^{J}$ y $f_{K L \ldots}^{J}$ están evaluadas en $h=0$. En lo sucesivo también evitaremos especificarlo en las derivadas de $g_{i}^{J}$ y $k_{i}^{J}$.

Teorema 4.2.3 Se satisface la siguiente relación

$$
\left(g_{i}^{J}\right)^{(q+1)}=(q+1) \sum_{t \in S N T_{q}} \alpha(t) \gamma(t) \sum_{j} a_{i j} \Phi_{j}(t) F^{J}(t)(y)
$$




$$
=(q+1) \sum_{t \in L S N T_{q}} \gamma(t) \sum_{j} a_{i j} \Phi_{j}(t) F^{J}(t)(y) .
$$

\section{Demostración:}

Para demostrar la relación anterior procederemos por inducción sobre $q$. La fórmula ha quedado probada hasta $q=3$. Aplicando (4.7) para derivar $g_{i}^{J}$ obtenemos que

$$
\left(g_{i}^{J}\right)^{(q+1)}=(q+1) q \sum_{j} a_{i j}\left(f^{J}\left(g_{j}\right)\right)^{(q-1)} .
$$

Ahora utilizaremos la fórmula de Faà di Bruno (véase Hairer et al. [54]) que nos proporciona la siguiente relación. Para $q \geq 1$ resulta

$$
\left.\left(f^{J}(g)\right)^{(q-1)}\right|_{h=0}=\sum_{t \in L S_{q}} \sum_{K_{1} \ldots K_{m}} f_{K_{1} \ldots K_{m}}^{J}(g)\left(g^{K_{1}}\right)^{\left(\delta_{1}\right)} \ldots\left(g^{K_{m}}\right)^{\left(\delta_{m}\right)} .
$$

donde $L S_{q}$ es el conjunto de todos los árboles con raíz y $q$ nodos que sólo tienen ramificaciones en la raíz, $m$ es el número de ramas que salen de la raíz y $\delta_{1}, \ldots, \delta_{m}$ son los números de nodos de cada rama, tales que $q=1+\delta_{1}+\cdots+\delta_{m}$.

Utilizando (4.9) e insertando las derivadas $\left(g_{j}^{K_{s}}\right)^{\left(\delta_{s}\right)}$ con $\delta_{s}<q$ obtenemos, tras reagrupar términos

$$
\begin{aligned}
\left(g_{i}^{J}\right)^{(q+1)}= & (q+1) q \sum_{t \in L S_{q}} \sum_{t_{1} \in L S N T_{\delta_{1}-1}} \cdots \sum_{t_{m} \in L S N T_{\delta_{m}-1}} \delta_{1} \cdots \delta_{m} \gamma\left(t_{1}\right) \cdots \gamma\left(t_{m}\right) . \\
& \sum_{j} a_{i j} \sum_{k_{1}} a_{j k_{1}} \Phi_{k_{1}}\left(t_{1}\right) \cdots \sum_{k_{m}} a_{j k_{m}} \Phi_{k_{m}}\left(t_{m}\right) \\
& \sum_{K_{1} \ldots K_{m}} f_{K_{1} \ldots K_{m}}^{J}(y) F^{K_{1}}\left(t_{1}\right)(y) \cdots F^{K_{m}}\left(t_{m}\right)(y) .
\end{aligned}
$$

La principal dificultad con que nos encontramos ahora es ver que a cada $(m+1)$-upla $\left(t, t_{1}, \ldots, t_{m}\right)$ con $t \in L S_{q}$ y $t_{s} \in L S N T_{\delta_{s}-1}$ le corresponde un árbol etiquetado $\tau \in L S N T_{q}$ tal que

$$
\begin{aligned}
\gamma(\tau) & =q \cdot \delta_{1} \cdots \delta_{m} \cdot \gamma\left(t_{1}\right) \cdots \gamma\left(t_{m}\right) \\
F^{J}(\tau)(y) & =\sum_{K_{1}, \ldots, K_{m}} f_{K_{1} \ldots K_{m}}^{J}(y) F^{K_{1}}\left(t_{1}\right)(y) \cdots F^{K_{m}}\left(t_{m}\right)(y) \\
\Phi_{j}(\tau) & =\sum_{k_{1}, \ldots, k_{m}} a_{j k_{1}} \ldots a_{j k_{m}} \Phi_{k_{1}}\left(t_{1}\right) \cdots \Phi_{k_{m}}\left(t_{m}\right)
\end{aligned}
$$


Esta correspondencia puede verse considerando que el árbol $\tau$ se obtiene si las ramas de $t$ se reeemplazan por los árboles $t_{1}, \ldots, t_{m}$ y las correspondientes etiquetas se toman de forma natural, por ejemplo, conservando el orden. De esta forma, todos los árboles $t \in L T_{q}$ aparecen exactamente una vez. Entonces, insertando (4.11)-(4.13) en (4.10) se obtiene el resultado buscado.

Teorema 4.2.4 Las derivadas de $k_{i}$ satisfacen la siguiente relación

$$
\left(k_{i}^{J}\right)^{(q-1)}=\frac{1}{q} \sum_{t \in S N T_{q}} \alpha(t) \gamma(t) \Phi_{j}(t) F^{J}(t)(y)=\frac{1}{q} \sum_{t \in L S N T_{q}} \gamma(t) \Phi_{j}(t) F^{J}(t)(y) .
$$

\section{Demostración:}

Teniendo en cuenta que $k_{i}^{J}=f^{J}\left(g_{i}\right)$, llevando (4.8) a (4.9) resulta

$$
\begin{aligned}
\left(f^{J}(g)\right)^{(q-1)=} & \sum_{t \in L S_{q}} \sum_{t_{1} \in L S N T_{\delta_{1}-1}} \cdots \sum_{t_{m} \in L S N T_{\delta_{m}-1}} \delta_{1} \cdots \delta_{m} \gamma\left(t_{1}\right) \cdots \gamma\left(t_{m}\right) . \\
& \sum_{k_{1}} a_{j k_{1}} \Phi_{k_{1}}\left(t_{1}\right) \cdots \sum_{k_{m}} a_{j k_{m}} \Phi_{k_{m}}\left(t_{m}\right) \\
& \sum_{K_{1} \ldots K_{m}} f_{K_{1} \ldots K_{m}}^{J}(y) F^{K_{1}}\left(t_{1}\right)(y) \cdots F^{K_{m}}\left(t_{m}\right)(y) .
\end{aligned}
$$

La misma correspondencia a la que hicimos referencia en el Teorema 4.2.3 nos permite concluir la demostración.

\subsubsection{Condiciones de orden. Error de truncación local}

En la presente sección aplicaremos la teoría de árboles anteriormente expuesta a los métodos de $\mathrm{RKN} h^{2}$ con el fin de encontrar las condiciones de orden para conseguir orden $p$. Así mismo, obtendremos una expresión para el error de truncación local en términos de las constantes de error que pueden calcularse gracias a los ya mencionados árboles especiales de Nyström con raíz.

Teorema 4.2.5 Un método $\mathrm{RKN} h^{2}$ es de orden $p$ cuando integra el problema (4.3) si se satisfacen las siguientes condiciones de orden:

$$
\sum_{i} \bar{b}_{i} \Phi_{i}(t)=\frac{1}{(\rho(t)+1) \gamma(t)}, \quad \forall t \in S N T_{\rho}, \quad \rho(t) \leq p-1
$$




$$
\begin{aligned}
& \sum \bar{b}_{i}^{*} \Phi_{i}(t)=0, \quad \forall t \in S N T_{\rho}, \quad \rho(t) \leq p-3, \\
& \sum_{i} b_{i} \Phi_{i}(t)=\frac{1}{\gamma(t)}, \quad \forall t \in S N T_{\rho}, \quad \rho(t) \leq p, \\
& \sum_{i} b_{i}^{*} \Phi_{i}(t)=0, \quad \forall t \in S N T_{\rho}, \quad \rho(t) \leq p-2 .
\end{aligned}
$$

\section{Demostración:}

Según la Definición 2.3.5, para que un método $\mathrm{RKN} h^{2}$ sea de orden $p$ es preciso que, tanto la solución exacta como su derivada, coincidan con las correspondientes aproximaciones numéricas hasta el término en $h^{p}$.

Aplicando el Teorema 4.2.2 al desarrollo de Taylor de la solución exacta se obtiene

$$
\begin{aligned}
& y^{J}\left(x_{0}+h\right)= \\
& =y_{0}^{J}+h y_{0}^{\prime J}+h^{2} \sum_{j=0}^{\infty} \frac{h^{j}}{(j+2) !} f_{0}^{(j)} \\
& =y_{0}^{J}+h y_{0}^{\prime J}+h^{2} \sum_{j=0}^{p-2} \frac{h^{j}}{(j+2) !} \sum_{t \in S N T_{j+1}} \alpha(t) F^{J}(t)\left(y_{0}\right)+O\left(h^{p+1}\right) .
\end{aligned}
$$

Según (4.4) para alcanzar orden $p$ bastará entonces obtener el desarrollo en serie de Taylor en $h=0$ de $\sum_{i=1}^{s}\left(\bar{b}_{i}+h^{2}\left(\omega^{J}\right)^{2} \bar{b}_{i}^{*}\right) k_{i}^{J}$ e identificar términos semejantes para $j=0, \ldots, p-2$.

$$
\begin{aligned}
& \sum_{i}\left(\bar{b}_{i}+h^{2}\left(\omega^{J}\right)^{2} \bar{b}_{i}^{*}\right) k_{i}^{J}= \\
& \sum_{j=0}^{p-2} \frac{h^{j}}{j !}\left\{\left(\sum_{i} \bar{b}_{i} k_{i}^{J}\right)^{(j)}+\left(\omega^{J}\right)^{2}\left(h^{2} \sum_{i} \bar{b}_{i}^{*} k_{i}^{J}\right)^{(j)}\right\}+O\left(h^{p-1}\right) .
\end{aligned}
$$

Aplicando el Teorema 4.2.4

$$
\left(\sum_{i} \bar{b}_{i} k_{i}^{J}\right)^{(j)}=\frac{1}{j+1} \sum_{i} \bar{b}_{i} \sum_{t \in S N T_{j+1}} \alpha(t) \gamma(t) \Phi_{i}(t) F^{J}(t)\left(y_{0}\right) .
$$


Para obtener el segundo término, aplicamos primero (4.7) y de nuevo el resultado del Teorema 4.2.4:

$$
\begin{aligned}
\left(h^{2} \sum_{i} \bar{b}_{i}^{*} k_{i}^{J}\right)^{(j)} & =j(j-1)\left(\sum_{i} \bar{b}_{i}^{*} k_{i}^{J}\right)^{(j-2)} \\
& =j(j-1) \sum_{i} \bar{b}_{i}^{*} \frac{1}{j-1} \sum_{t \in S N T_{j-1}} \alpha(t) \gamma(t) \Phi_{i}(t) F^{J}(t)\left(y_{0}\right) \\
& =j \sum_{i} \bar{b}_{i}^{*} \sum_{t \in S N T_{j-1}} \alpha(t) \gamma(t) \Phi_{i}(t) F^{J}(t)\left(y_{0}\right) .
\end{aligned}
$$

Sustituyendo los resultados anteriores en (4.18) se tiene

$$
\begin{aligned}
& \sum_{i}\left(\bar{b}_{i}+h^{2}\left(\omega^{J}\right)^{2} \bar{b}_{i}^{*}\right) k_{i}^{J}= \\
& \sum_{j=0}^{p-2} h^{j}\left\{\frac{1}{(j+1) !} \sum_{i} \bar{b}_{i} \sum_{t \in S N T_{j+1}} \alpha(t) \gamma(t) \Phi_{i}(t) F^{J}(t)\left(y_{0}\right)\right. \\
& \left.+\frac{\left(\omega^{J}\right)^{2}}{(j-1) !} \sum_{i} \bar{b}_{i}^{*} \sum_{t \in S N T_{j-1}} \alpha(t) \gamma(t) \Phi_{i}(t) F^{J}(t)\left(y_{0}\right)\right\}+O\left(h^{p-1}\right) .
\end{aligned}
$$

Análogamente, la derivada de la solución exacta se puede expresar

$$
y^{\prime J}\left(x_{0}+h\right)=y_{0}^{\prime J}+h \sum_{j=0}^{p-1} \frac{h^{j}}{(j+1) !} \sum_{t \in S N T_{j+1}} \alpha(t) F^{J}(t)\left(y_{0}\right)+O\left(h^{p+1}\right) .
$$

Siguiendo el razonamiento anterior para buscar orden $p$ bastará con desarrollar ahora $\sum_{i=1}^{s}\left(b_{i}+h^{2}\left(\omega^{J}\right)^{2} b_{i}^{*}\right) k_{i}^{J}$ en serie de Taylor en $h=0$ :

$$
\begin{aligned}
& \sum_{i=1}^{s}\left(b_{i}+h^{2}\left(\omega^{J}\right)^{2} b_{i}^{*}\right) k_{i}^{J}= \\
& \quad \sum_{j=0}^{p-1} h^{j}\left\{\frac{1}{(j+1) !} \sum_{i} b_{i} \sum_{t \in S N T_{j+1}} \alpha(t) \gamma(t) \Phi_{i}(t) F^{J}(t)\left(y_{0}\right)\right. \\
& \left.+\frac{\left(\omega^{J}\right)^{2}}{(j-1) !} \sum_{i} b_{i}^{*} \sum_{t \in S N T_{j-1}} \alpha(t) \gamma(t) \Phi_{i}(t) F^{J}(t)\left(y_{0}\right)\right\}+O\left(h^{p-1}\right) .
\end{aligned}
$$

Identificando términos semejantes, tanto en la solución exacta como en la derivada de la misma, con los desarrollos obtenidos para las 
aproximaciones, llegamos a los sistemas

$$
\frac{\gamma(t)}{(j+1) !} \sum_{i} \bar{b}_{i} \Phi_{i}(t)=\frac{1}{(j+2) !}
$$

para $t \in S N T_{j+1}$ con $j=0, \ldots, p-2$,

$$
\sum \bar{b}_{i}^{*} \Phi_{i}(t)=0
$$

para $t \in S N T_{j-1}$ con $j=0, \ldots, p-2$,

$$
\gamma(t) \sum_{i} b_{i} \Phi_{i}(t)=1
$$

para $t \in S N T_{j+1}$ con $j=0, \ldots, p-1$,

$$
\sum_{i} b_{i}^{*} \Phi_{i}(t)=0
$$

para $t \in S N T_{j-1}$ con $j=0, \ldots, p-1$.

Teniendo en cuenta que $\rho(t)=j+1$ para $t \in S N T_{j+1}$, de forma inmediata se obtienen las condiciones de orden buscadas.

Teorema 4.2.6 Consideremos un método $\mathrm{RKN} h^{2}$ de orden $p$ con $f$ suficientemente regular, el error de truncación local cometido es

$$
\begin{aligned}
y_{1}^{J}-y^{J}\left(x_{0}+h\right) & =h^{p+1}\left\{\sum_{t \in S N T_{p}} \tau^{(p)} F^{J}(t)\left(y_{0}\right)\right. \\
& \left.+\left(\omega^{J}\right)^{2} \sum_{t \in S N T_{p-2}} \tau^{*(p-2)} F^{J}(t)\left(y_{0}\right)\right\}+O\left(h^{p+2}\right), \\
y_{1}^{\prime J}-y^{\prime J}\left(x_{0}+h\right) & =h^{p+1}\left\{\sum_{t \in S N T_{p+1}} \tau^{\prime(p+1)} F^{J}(t)\left(y_{0}\right)\right. \\
& \left.+\left(\omega^{J}\right)^{2} \sum_{t \in S N T_{p-1}} \tau^{\prime *(p-1)} F^{J}(t)\left(y_{0}\right)\right\}+O\left(h^{p+2}\right)
\end{aligned}
$$

donde

$$
\tau^{(p)}=\frac{\alpha(t) \gamma(t)}{p !}\left(\sum_{i} \bar{b}_{i} \Phi_{i}(t)-\frac{1}{(p+1) \gamma(t)}\right), \quad t \in S N T_{p}
$$




$$
\begin{aligned}
\tau^{*(p-2)} & =\frac{\alpha(t) \gamma(t)}{(p-2) !} \sum_{i} \bar{b}_{i}^{*} \Phi_{i}(t), \quad t \in S N T_{p-2}, \\
\tau^{\prime(p+1)} & =\frac{\alpha(t) \gamma(t)}{(p+1) !}\left(\sum_{i} b_{i} \Phi_{i}(t)-\frac{1}{\gamma(t)}\right), \quad t \in S N T_{p+1} \\
\tau^{\prime(p-1)} & =\frac{\alpha(t) \gamma(t)}{(p-1) !} \sum_{i} b_{i}^{*} \Phi_{i}(t), \quad t \in S N T_{p-1} .
\end{aligned}
$$

\section{Demostración:}

Usando los desarrollos de Taylor obtenidos previamente, podemos escribir $y_{1}^{J}-y^{J}\left(x_{0}+h\right)$ e $y_{1}^{\prime J}-y^{\prime J}\left(x_{0}+h\right)$ de la siguiente forma:

$$
\begin{aligned}
y_{1}^{J}-y^{J}\left(x_{0}+h\right) & =\sum_{j=2}^{\infty} h^{j}\left\{\sum_{t \in S N T_{j-1}} \tau^{(j-1)} F^{J}(t)\left(y_{0}\right)\right. \\
& \left.+\left(\omega^{J}\right)^{2} \sum_{t \in S N T_{j-3}} \tau^{*(j-3)} F^{J}(t)\left(y_{0}\right)\right\}+O\left(h^{p+2}\right), \\
y_{1}^{\prime J}-y^{\prime J}\left(x_{0}+h\right) & =\sum_{j=1}^{\infty} h^{j}\left\{\sum_{t \in S N T_{j}} \tau^{\prime(j)} F^{J}(t)\left(y_{0}\right)\right. \\
& \left.+\left(\omega^{J}\right)^{2} \sum_{t \in S N T_{j-2}} \tau^{*(j-2)} F^{J}(t)\left(y_{0}\right)\right\}+O\left(h^{p+2}\right),
\end{aligned}
$$

donde

$$
\begin{aligned}
\tau^{(j-1)} & =\frac{\alpha(t) \gamma(t)}{(j-1) !}\left(\sum_{i} \bar{b}_{i} \Phi_{i}(t)-\frac{1}{j \gamma(t)}\right), \quad t \in S N T_{j-1} \\
\tau^{*(j-3)} & =\frac{\alpha(t) \gamma(t)}{(j-3) !} \sum_{i} \bar{b}_{i}^{*} \Phi_{i}(t), \quad t \in S N T_{j-3}, \\
\tau^{\prime(j)} & =\frac{\alpha(t) \gamma(t)}{j !}\left(\sum_{i} b_{i} \Phi_{i}(t)-\frac{1}{\gamma(t)}\right), \quad t \in S N T_{j} \\
\tau^{\prime *(j-2)} & =\frac{\alpha(t) \gamma(t)}{(j-2) !} \sum_{i} b_{i}^{*} \Phi_{i}(t), \quad t \in S N T_{j-2} .
\end{aligned}
$$

tomando $\tau^{*(-1)}=\tau^{*(0)}=\tau^{*(-2)}=\tau^{*(-1)}=0$.

Los coeficientes $\tau^{(j)}, \tau^{\prime(j)}, \tau^{*(j)}$ y $\tau^{\prime *(j)}$ se denominan constantes de error. Anulando los términos en $h^{j}$ con $j=0, \ldots, p$ en (4.19) y (4.20), se obtienen las expresiones del teorema. 


\subsection{5 Árboles para un oscilador no perturbado. Condiciones de orden}

Consideremos de nuevo el problema de un oscilador armónico no perturbado:

$$
\left(y^{J}\right)^{\prime \prime}+\left(\omega^{J}\right)^{2} y^{J}=0 .
$$

En este caso $f^{J}=-\left(\omega^{J}\right)^{2} y^{J}$ y se cumple

$$
\begin{aligned}
f_{K}^{J} & =0, \quad \text { si } J \neq K, \\
f_{J}^{J} & =-\left(\omega^{J}\right)^{2}, \\
f_{K L M \ldots}^{J} & =0, \quad \forall J, K, L, M \ldots
\end{aligned}
$$

De esta forma los árboles especiales de Nyström se simplifican considerablemente, quedando un único árbol para cada orden q. Dicho árbol, que denotaremos por $t^{q}$, es el que sólo tiene una ramificación por cada nodo. Las correspondientes diferenciales elementales serán entonces:

$$
\begin{aligned}
f^{J} & =-\left(\omega^{J}\right)^{2} y^{J} \\
\sum_{K} f_{K}^{J} y^{\prime K} & =-\left(\omega^{J}\right)^{2} y^{\prime J}, \\
\sum_{K} f_{K}^{J} f^{K} & =\left(\omega^{J}\right)^{4} y^{J} \\
\sum_{K L} f_{K}^{J} f_{L}^{K} y^{\prime L} & =\left(\omega^{J}\right)^{4} y^{\prime}, \\
& \ldots
\end{aligned}
$$

Es sencillo comprobar mediante un razonamiento inductivo que

$$
\begin{aligned}
F^{J}\left(t^{2 n}\right)(y) & =(-1)^{n}\left(\omega^{J}\right)^{2 n} y^{J}, \\
F^{J}\left(t^{2 n-1}\right)(y) & =(-1)^{n}\left(\omega^{J}\right)^{2 n} y^{J} .
\end{aligned}
$$

y por lo tanto se satisface la siguiente relación:

$$
F^{J}\left(t^{q}\right)(y)=-\left(\omega^{J}\right)^{2} F^{J}\left(t^{q-2}\right)(y) .
$$

Como veremos en los lemas enunciados a continuación, para los árboles $t^{q}$ las funciones $\alpha\left(t^{q}\right)$ y $\gamma\left(t^{q}\right)$ son sencillas y esto simplificará, como veremos posteriormente, las expresiones de las condiciones de orden. 
Lema 4.2.7 Para todo árbol $t^{q}$ con $q \geq 1$, se satisface la siguiente relación

$$
\gamma\left(t^{q}\right)=q !
$$

\section{Demostración:}

Por definición, un árbol $t^{q}$ es aquel con $q$ nodos que tiene sólo una ramificación por nodo. Para calcular $\gamma\left(t^{q}\right)$ basta multiplicar $\rho\left(t^{q}\right)=q$ por los órdenes de los árboles que aparecen si las raíces, una detrás de otra se van quitando de $t^{q}$, y así resultarían sucesivamente $t^{q-1}, t^{q-2}, \ldots, t^{1}$. Entonces, es claro que

$$
\gamma\left(t^{q}\right)=q \cdot(q-1) \cdot(q-2) \cdots 1=q !
$$

y el resultado quedaría demostrado.

Lema 4.2.8 Para todo árbol $t^{q}$ con $q \geq 1$, se tiene

$$
\alpha\left(t^{q}\right)=1 .
$$

\section{Demostración:}

Por construcción, los árboles con una sola ramificación por nodo sólo aparecen una vez por cada orden $q$ y, por tanto, $\alpha\left(t^{q}\right)=1$.

Teorema 4.2.9 Un método $\mathrm{RKN} h^{2}$ es de orden $p$ cuando se integra el problema (4.21), si se satisfacen las siguientes condiciones de orden

$$
\begin{aligned}
\sum_{i} \bar{b}_{i}^{*} \Phi\left(t^{j-2}\right) & =\sum_{i} \bar{b}_{i} \Phi\left(t^{j}\right)-\frac{1}{(j+1) !}, \quad j=1, \ldots, p-1, \\
\sum_{i} b_{i}^{*} \Phi\left(t^{j-2}\right) & =\sum_{i} b_{i} \Phi\left(t^{j}\right)-\frac{1}{j !}, \quad j=1, \ldots, p .
\end{aligned}
$$

\section{Demostración:}

Como ya hemos mencionado con anterioridad, si consideramos el problema del oscilador armónico, para cada conjunto de árboles $S N T_{j}$ con $j=1 \cdots p$ sólo resulta un árbol, $t^{j}$. Por lo tanto, si sustituimos la relación (4.22) en (4.19) y (4.20), cuando un método RKNh $h^{2}$ integra 
el problema (4.21) se tiene lo siguiente

$$
\begin{aligned}
y_{1}^{J}-y^{J}\left(x_{0}+h\right) & =\sum_{j=2}^{\infty} h^{j}\left(\tau_{o s c}^{(j-1)}-\tau_{o s c}^{*(j-3)}\right) F^{J}\left(t^{j-1}\right)\left(y_{0}\right), \\
y_{1}^{\prime J}-y^{\prime J}\left(x_{0}+h\right) & =\sum_{j=1}^{\infty} h^{j}\left(\tau_{o s c}^{\prime(j)}-\tau_{o s c}^{\prime *(j-2)}\right) F^{J}\left(t^{j}\right)\left(y_{0}\right)
\end{aligned}
$$

donde

$$
\begin{aligned}
\tau_{\text {osc }}^{(j-1)} & =\frac{\alpha\left(t^{j-1}\right) \gamma\left(t^{j-1}\right)}{(j-1) !}\left(\sum_{i} \bar{b}_{i} \Phi_{i}\left(t^{j-1}\right)-\frac{1}{j \gamma\left(t^{j-1}\right)}\right) \\
\tau_{\text {osc }}^{*(j-3)} & =\frac{\alpha\left(t^{j-3}\right) \gamma\left(t^{j-3}\right)}{(j-3) !} \sum_{i} \bar{b}_{i}^{*} \Phi_{i}\left(t^{j-3}\right), \\
\tau_{\text {osc }}^{\prime(j)} & =\frac{\alpha\left(t^{j}\right) \gamma\left(t^{j}\right)}{j !}\left(\sum_{i} b_{i} \Phi_{i}\left(t^{j}\right)-\frac{1}{\gamma\left(t^{j}\right)}\right) \\
\tau_{\text {osc }}^{*(j-2)} & =\frac{\alpha\left(t^{j-2}\right) \gamma\left(t^{j-2}\right)}{(j-2) !} \sum_{i} b_{i}^{*} \Phi_{i}\left(t^{j-2}\right) .
\end{aligned}
$$

Entonces, para obtener un método $\mathrm{RKN} h^{2}$ de orden $p$ para este problema particular las condiciones de orden que se han de satisfacer son las que siguen:

$$
\begin{aligned}
\tau_{o s c}^{(j)}-\tau_{o s c}^{*(j-2)} & =0, \quad j=1, \ldots, p-1, \\
\tau_{o s c}^{\prime(j)}-\tau_{o s c}^{\prime *(j-2)} & =0, \quad j=1, \ldots, p .
\end{aligned}
$$

Sustituyendo en las ecuaciones anteriores los resultados de los Lemas 4.2.7 y 4.2.8 se obtienen directamente las condiciones de orden del teorema.

Teorema 4.2.10 El error de truncación local para un método RKN $h^{2}$ de orden $p$ cuando se integra el problema (4.21) es

$$
\begin{aligned}
y_{1}^{J}-y^{J}\left(x_{0}+h\right) & =h^{p+1}\left(\tau_{o s c}^{(p)}-\tau_{o s c}^{*(p-2)}\right) F^{J}\left(t^{p}\right)\left(y_{0}\right)+O\left(h^{p+2}\right), \\
y_{1}^{\prime J}-y^{\prime J}\left(x_{0}+h\right) & =h^{p+1}\left(\tau_{\text {osc }}^{\prime(p+1)}-\tau_{\text {osc }}^{\prime *(p-1)}\right) F^{J}\left(t^{p+1}\right)\left(y_{0}\right)+O\left(h^{p+2}\right),
\end{aligned}
$$

donde

$$
\tau_{\text {osc }}^{(p)}=\sum_{i} \bar{b}_{i} \Phi_{i}\left(t^{p}\right)-\frac{1}{(p+1) !}
$$




$$
\begin{aligned}
\tau_{\text {osc }}^{*(p-2)} & =\sum_{i} \bar{b}_{i}^{*} \Phi_{i}\left(t^{p-2}\right), \\
\tau_{\text {osc }}^{(p+1)} & =\sum_{i} b_{i} \Phi_{i}\left(t^{p+1}\right)-\frac{1}{(p+1) !} \\
\tau_{\text {osc }}^{*(p-1)} & =\sum_{i} b_{i}^{*} \Phi_{i}\left(t^{p-1}\right) .
\end{aligned}
$$

\section{Demostración:}

Es una consecuencia inmediata de imponer las condiciones de orden del Teorema 4.2 .9 en las expresiones (4.23) y (4.24)

\subsubsection{Condiciones de orden para métodos $\mathbf{R K N} h^{2} p: q$}

El siguiente teorema nos proporciona las condiciones de orden para obtener métodos del tipo $\mathrm{RKN} h^{2}$ que integren un problema general con orden $p$ y además alcancen orden oscilatorio $q$. Como ya hemos comentado en diversas ocasiones a lo largo de la memoria, este aumento de orden para el problema del oscilador no perturbado se traducirá en un buen comportamiento de estos métodos cuando integren problemas oscilatorios.

Teorema 4.2.11 Las condiciones de orden para obtener un método $\mathrm{RKN} h^{2} p: q$, con $p<q$, son las que siguen:

$$
\begin{aligned}
\sum_{i} \bar{b}_{i} \Phi_{i}(t) & =\frac{1}{(\rho(t)+1) \gamma(t)}, \quad \forall t \in S N T_{\rho}, \quad \rho(t) \leq p-1,(4) \\
\sum_{i} \bar{b}_{i}^{*} \Phi_{i}(t) & =0, \quad \forall t \in S N T_{\rho}, \quad \rho(t) \leq p-3 \\
\sum_{i} b_{i} \Phi_{i}(t) & =\frac{1}{\gamma(t)}, \quad \forall t \in S N T_{\rho}, \quad \rho(t) \leq p \\
\sum_{i} b_{i}^{*} \Phi_{i}(t) & =0, \quad \forall t \in S N T_{\rho}, \quad \rho(t) \leq p-2 \\
\sum_{i} \bar{b}_{i}^{*} \Phi\left(t^{j-2}\right) & =\sum_{i} \bar{b}_{i} \Phi\left(t^{j}\right)-\frac{1}{(j+1) !}, \quad j=p, \ldots, q-1 \\
\sum_{i} b_{i}^{*} \Phi\left(t^{j-2}\right) & =\sum_{i} b_{i} \Phi\left(t^{j}\right)-\frac{1}{j !}, \quad j=p+1, \ldots, q
\end{aligned}
$$




\section{Demostración:}

Basta aplicar los Teoremas 4.2.5 y 4.2.9 que anulan los términos hasta $h^{p}$ en el error de truncación local para una función $f$ general, y los términos hasta $h^{q}$ cuando se integra un oscilador no perturbado. Obviamente, los términos en $h^{j}$ con $j=0, \ldots, p$ del error de truncación local se anulan también en este caso al imponer las condiciones generales de orden $p$ y sólo es necesario satisfacer las condiciones del Teorema 4.2 .9 para $j=p, \ldots, q-1$ o $j=p+1, \ldots, q$ según corresponda.

Nota 4.2.12 Un caso particular de problema oscilatorio es el ya mencionado oscilador perturbado, para el que es muy sencillo obtener una expresión para el error de truncación local para un método $\mathrm{RKN}^{2} p: q$, sin más que tener en cuenta que la función que corresponde a dicho problema, $f^{J}=\varepsilon^{J} g^{J}-\left(\omega^{J}\right)^{2} y^{J}$, consta de dos sumandos, el segundo de los cuales es la función correspondiente al oscilador no perturbado. Bastará considerar (4.19) y (4.20) para la parte perturbada y el Teorema 4.2.10 para la no perturbada obteniendo finalmente la siguiente expresión:

$$
\begin{aligned}
y_{1}^{J}-y^{J}\left(x_{0}+h\right) & =\varepsilon^{J} \sum_{j=p+1}^{\infty} h^{j}\left\{\sum_{t \in S N T_{j-1}} \tau^{(j-1)} G^{J}(t)\left(y_{0}\right)\right. \\
& \left.+\left(\omega^{J}\right)^{2} \sum_{t \in S N T_{j-3}} \tau^{*(j-3)} G^{J}(t)\left(y_{0}\right)\right\} \\
& +\sum_{j=q+1}^{\infty} h^{j}\left(\tau_{o s c}^{(j-1)}-\tau_{o s c}^{*(j-3)}\right) F_{o s c}^{J}\left(t^{j-1}\right)\left(y_{0}\right), \\
y_{1}^{\prime J}-y^{\prime J}\left(x_{0}+h\right) & =\varepsilon^{J} \sum_{j=p+1}^{\infty} h^{j}\left\{\sum_{t \in S N T_{j}} \tau^{\prime(j)} G^{J}(t)\left(y_{0}\right)\right. \\
& \left.+\left(\omega^{J}\right)^{2} \sum_{t \in S N T_{j-2}} \tau^{\prime *(j-1)} G^{J}(t)\left(y_{0}\right)\right\} \\
& +\sum_{j=q+1}^{\infty} h^{j}\left(\tau_{o s c}^{\prime(j)}-\tau_{o s c}^{*(j-2)}\right) F_{o s c}^{J}\left(t^{j}\right)\left(y_{0}\right),
\end{aligned}
$$


donde

$$
\begin{aligned}
\tau^{(j-1)} & =\frac{\alpha(t)}{j !}\left(j \gamma(t) \sum_{i} \bar{b}_{i} \Phi_{i}(t)-1\right), \quad t \in S N T_{j-1}, \\
\tau^{*(j-3)} & =\frac{\alpha(t) \gamma(t)}{(j-3) !} \sum_{i} \bar{b}_{i}^{*} \Phi_{i}(t), \quad t \in S N T_{j-3}, \\
\tau^{\prime(j)} & =\frac{\alpha(t)}{(j) !}\left(\gamma(t) \sum_{i} b_{i} \Phi_{i}(t)-1\right), \quad t \in S N T_{j}, \\
\tau^{\prime *(j-1)} & =\frac{\alpha(t) \gamma(t)}{(j-2) !} \sum_{i} b_{i}^{*} \Phi_{i}(t), \quad t \in S N T_{j-1}, \\
\tau_{o s c}^{(j-1)} & =\frac{\alpha\left(t^{j-1}\right)}{j !}\left(j \gamma\left(t^{j-1}\right) \sum_{i} \bar{b}_{i} \Phi_{i}\left(t^{j-1}\right)-1\right), \\
\tau_{o s c}^{*(j-3)} & =\frac{\alpha\left(t^{j-3}\right) \gamma\left(t^{j-3}\right)}{(j-3) !} \sum_{i} \bar{b}_{i}^{*} \Phi_{i}\left(t^{j-3}\right), \\
\tau_{o s c}^{\prime(j)} & =\frac{\alpha\left(t^{j}\right)}{j !}\left(\gamma\left(t^{j-1}\right) \sum_{i} b_{i} \Phi_{i}\left(t^{j}\right)-1\right), \\
\tau_{\text {osc }}^{\prime *(j-2)} & =\frac{\alpha\left(t^{j-2}\right) \gamma\left(t^{j-2}\right)}{(j-2) !} \sum_{i} b_{i}^{*} \Phi_{i}\left(t^{j-2}\right),
\end{aligned}
$$

siendo $G^{J}(t)(y)$ las diferenciales elementales correspondientes a la función $g^{J}$ y $F_{o s c}^{J}\left(t^{j}\right)(y)$ las correspondientes a la parte no perturbada.

\subsubsection{Condiciones simplificadoras}

Para obtener un método $\mathrm{RKN} h^{2} p: q$ con órdenes elevados se requiere resolver un sistema con un gran número de ecuaciones. Afortunadamente no todas ellas son independientes, con lo que el número final de ecuaciones a satisfacer para imponer un determinado orden $p$, se reduce notablemente. Las condiciones simplificadoras que emplearemos para obtener métodos de orden alto ya han sido ampliamente utilizadas por otros autores tales como Calvo [7], Calvo y Sanz-Serna [8], Dormand et al. [20, 21], González [44], González et al. [50], Hairer [52, 53], Hairer y Wanner [55] o Hairer et al. [54]. Estas condiciones son las que se obtienen de los lemas que presentamos a continuación. 
Lema 4.2.13 $\mathrm{Si}$

$$
\bar{b}_{i}=b_{i}\left(1-c_{i}\right) \quad i=1, \ldots, s,
$$

las condiciones (4.25) implican (4.27). 


\section{Demostración:}

Sea $t \in S N T_{\rho}$ con orden $\rho(t) \leq p-1$. Sea $u \in S N T_{\rho}$ con orden $\rho(u)=\rho(t)+1$ obtenido al añadir a $t$ un hijo delgado a la raíz de $t$. Como ya indicamos en la definición de $\Phi_{i}$, la expresión $c_{k}^{m}$ se obtiene cuando un nodo grueso $k$ tiene $m$ hijos delgados finales. Por tanto, para los árboles $t$ y $u$ se cumple que $\Phi_{i}(u)=c_{i} \Phi_{i}(t)$. Además, teniendo en cuenta (4.11), es fácil ver que $\gamma(u)=(\rho(t)+1) \gamma(t) / \rho(t)$. Entonces, bajo la suposición del enunciado la conclusión es clara

$$
\sum_{i=1}^{s} \bar{b}_{i} \Phi_{i}(t)=\sum_{i=1}^{s} b_{i} \Phi_{i}(t)-\sum_{i=1}^{s} b_{i} \Phi_{i}(u)=\frac{1}{\gamma(t)}-\frac{1}{\gamma(u)}=\frac{1}{(\rho(t)+1) \gamma(t)} .
$$

Nota 4.2.14 Gracias al lema anterior, para encontrar los coeficientes de un método RKN resolviendo el sistema correspondiente a las condiciones de orden para los coeficientes $b_{i}$ podemos obtener los coeficientes $\bar{b}_{i}$ a partir de las relaciones del Lema 4.2.13.

Lema 4.2.15 Sean $t, u \in S N T$ como los de la Figura 4.2, donde las partes dentro del círculo se suponen idénticas. Entonces, si

$$
\sum_{j=1}^{i-1} a_{i j}=\frac{c_{i}^{2}}{2} \quad i=2, \ldots, s
$$

las condiciones de orden para $t$ y $u$ son las mismas.

\section{Demostración:}

A partir de la definición de $\Phi_{i}(t)$ se sigue que $\Phi_{i}(t)=\Phi_{i}(u) / 2$ y considerando (4.11) se llega a que $\gamma(t)=2 \gamma(u)$. Además ambos árboles tienen el mismo orden. Como consecuencia, bajo la suposición del enunciado, se obtienen condiciones de orden equivalentes.

Lema 4.2.16 Sean $t, u \in S N T$ como los de la Figura 4.3, donde las partes dentro de los círculos se suponen idénticas. Entonces, si

$$
\sum_{j=1}^{i-1} a_{i j} c_{j}=\frac{c_{i}^{3}}{6} \quad i=3, \ldots, s
$$

las condiciones de orden para $t$ y $u$ son las mismas. 


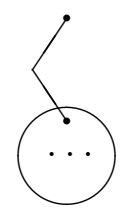

$t$

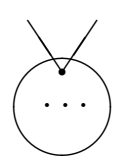

$u$

Figura 4.2: $S N T$ del Lema 4.2.15.

\section{Demostración:}

Siguiendo la demostración del Teorema 4.2 .15 se tiene que $\Phi_{i}(t)=$ $\Phi_{i}(u) / 6$ y $\gamma(t)=6 \gamma(u)$. A partir de esto la demostración del teorema es inmediata.
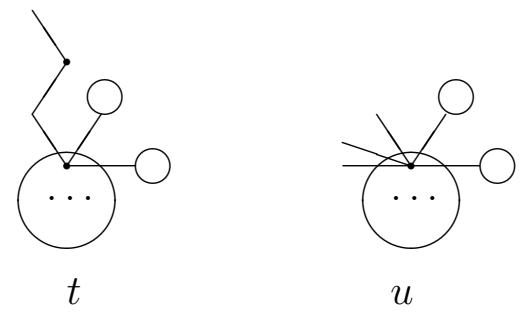

Figura 4.3: $S N T$ del Lema 4.2.16.

Lema 4.2.17 Sean $t, u \in S N T$ como los de la Figura 4.4, donde las partes dentro de los círculos se suponen idénticas. Entonces, si

$$
\sum_{j=1}^{i-1} a_{i j} c_{j}^{2}=\frac{c_{i}^{4}}{12} \quad i=3, \ldots, s,
$$

las condiciones de orden para $t$ y $u$ son las mismas.

Demostración:

Siguiendo la demostración del Teorema 4.2 .15 se tiene que $\Phi_{i}(t)=$ $\Phi_{i}(u) / 12$ y $\gamma(t)=12 \gamma(u)$. Y de forma trivial se obtiene el resultado del teorema. 


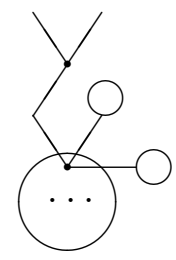

$t$

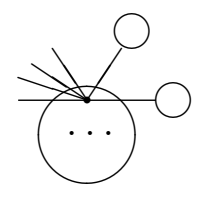

$u$

Figura 4.4: SNT del Lema 4.2.17.

Nota 4.2.18 Para órdenes elevados puede ser útil para la generalización de los lemas anteriores,

$$
\sum_{j=1}^{i-1} a_{i j} c_{j}^{q}=\frac{c_{i}^{q+2}}{(q+2)(q+1)}
$$

para $q>2$ (véase Hairer [52, 53], Hairer et al. [54] y Hairer y Wanner [55] para más detalles).

Las condiciones de los lemas 4.2.15, 4.2.16 y 4.2.17 no se pueden aplicar directamente cuando se trata de un método explícito debido a que ciertos coeficientes son nulos por la propia definición de método explícito. En ese caso los lemas anteriores se aplican bajo algunas condiciones adicionales como se expone en los lemas que siguen (véase Hairer $[52,53])$.

Lema 4.2.19 Sean $t, u \in S N T$ como los de la Figura 4.5, donde las partes dentro de los círculos se suponen idénticas. Entonces, si

$$
\sum_{j=1}^{i-1} a_{i j} c_{j}=\frac{c_{i}^{3}}{6} \quad i=3, \ldots, s,
$$

y $b_{2}=0$, las condiciones de orden para $t$ y $u$ son las mismas.

Lema 4.2.20 Sean $t, u \in S N T$ como los de la Figura 4.6, donde las partes dentro de los círculos se suponen idénticas. Entonces, si

$$
\sum_{j=1}^{i-1} a_{i j} c_{j}^{2}=\frac{c_{i}^{4}}{12} \quad i=3, \ldots, s,
$$

y $b_{2}=0$, las condiciones de orden para $t$ y $u$ son las mismas. 


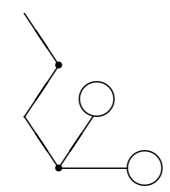

$t$

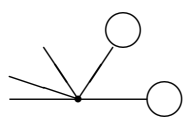

$u$

Figura 4.5: SNT del Lema 4.2.19.

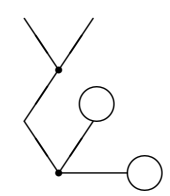

$t$

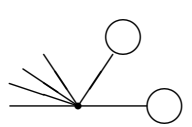

$u$

Figura 4.6: $S N T$ del Lema 4.2.20.

Lema 4.2.21 Sean $t, u \in S N T$ como los de la Figura 4.7 con $k+1$ ramas saliendo de la raíz y con idénticas partes dentro de los círculos. Entonces, si

$$
\sum_{j=1}^{i-1} a_{i j} c_{j}=\frac{c_{i}^{3}}{6} \quad i=3, \ldots, s
$$

y

$$
\sum_{i=3}^{s} b_{i} c_{i}^{k} a_{i 2}=0
$$

con $k=0,1,2$, las condiciones de orden para $t$ y $u$ son las mismas.

Lema 4.2.22 Sean $t, u \in S N T$ como los de la Figura 4.8 con $k+1$ ramas saliendo de la raíz y con idénticas partes dentro de los círculos. Entonces, si

$$
\sum_{j=1}^{i-1} a_{i j} c_{j}^{2}=\frac{c_{i}^{4}}{12} \quad i=3, \ldots, s
$$



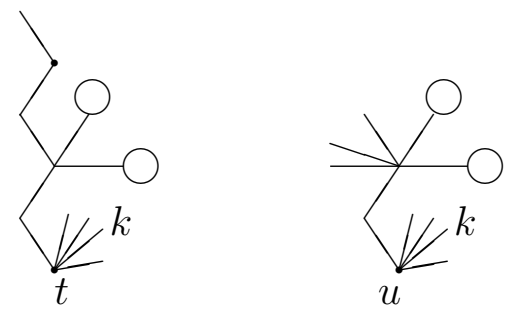

Figura 4.7: SNT del Lema 4.2.21.

$\mathrm{y}$

$$
\sum_{i=3}^{s} b_{i} c_{i}^{k} a_{i 2}=0
$$

con $k=0,1,2$, las condiciones de orden para $t$ y $u$ son las mismas.
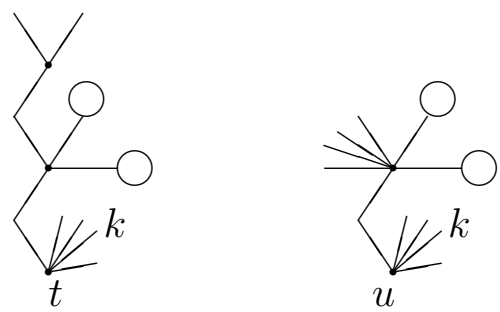

Figura 4.8: $S N T$ del Lema 4.2.22.

Utilizaremos además las condiciones simplificadoras que nos proporciona el siguiente lema que se emplean con frecuencia en la obtención de métodos Runge-Kutta-Nyström de orden elevado (véase Hairer $[52,53])$.

Lema 4.2.23 Sean $t, u, v, w \in S N T$ como los de la Figura 4.9. Entonces, si

$$
\sum_{i=j+1}^{s} b_{i} a_{i j}=b_{j}\left(\frac{c_{j}^{2}}{2}-c_{j}+\frac{1}{2}\right) \text {, }
$$


con $j=1, \ldots, s-1$ y se cumplen las condiciones de orden para los árboles $t, u$ y $v$, entonces se satisfacen también las condiciones de orden correspondientes al árbol $w$.

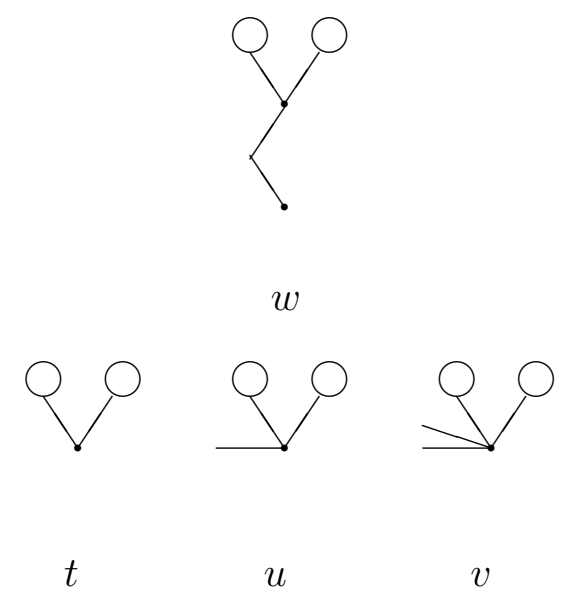

Figura 4.9: $S N T$ del Lema 4.2.23.

\section{Demostración:}

Antes de probar el resultado del teorema establezcamos algunas relaciones entre los árboles $t, u, v$ y $w$. Llamemos $p$ al orden del árbol $t$, entonces es claro que

$$
\rho(u)=p+1, \quad \rho(v)=p+2, \quad \rho(w)=p+2 .
$$

La función densidad de $t$ la podemos escribir como $\gamma(t)=p \cdot c$, donde $c$ representa el producto de los órdenes de todos los árboles que aparecen si las raíces, una detrás de otra, se van quitando en $t$. Como consecuencia, se tiene para los árboles $u, v$ y $w$

$$
\begin{aligned}
\gamma(u) & =(p+1) \cdot c, \\
\gamma(v) & =(p+2) \cdot c, \\
\gamma(w) & =(p+2) \cdot(p+1) \cdot p \cdot c .
\end{aligned}
$$


Teniendo en cuenta este resultado, las condiciones de orden para los árboles considerados se pueden escribir como sigue

$$
\begin{aligned}
\sum_{j} b_{j} \Phi_{j}(t) & =\frac{1}{p \cdot c}, \\
\sum_{j} b_{j} \Phi_{j}(u) & =\frac{1}{(p+1) \cdot c}, \\
\sum_{j} b_{j} \Phi_{j}(v) & =\frac{1}{(p+2) \cdot c}, \\
\sum_{j} b_{j} \Phi_{j}(w) & =\frac{1}{(p+2) \cdot(p+1) \cdot p \cdot c} .
\end{aligned}
$$

Por otro lado, si $\Phi_{j}(t)$ es la función peso elementalpara el árbol $t$ es sencillo ver que

$$
\begin{aligned}
\Phi_{j}(u) & =c_{j} \Phi_{j}(t), \\
\Phi_{j}(v) & =c_{j}^{2} \Phi_{j}(t), \\
\Phi_{j}(w) & =\sum_{k} a_{j k} \Phi_{k}(t) .
\end{aligned}
$$

Tras multiplicar la expresión (4.31) por $\Phi_{j}(t)$ realizamos la suma en $j$. Teniendo en cuenta (4.36) y (4.37), y que se satisfacen las condiciones de orden (4.32), (4.33) y (4.34) llegamos a

$$
\begin{aligned}
\sum_{j} \sum_{i=j+1}^{s} b_{i} a_{i j} \Phi_{j}(t) & =\frac{1}{2} \frac{1}{(p+2) \cdot c}-\frac{1}{(p+1) \cdot c}+\frac{1}{2} \frac{1}{p \cdot c}= \\
& =\frac{1}{(p+2) \cdot(p+1) \cdot p \cdot c} .
\end{aligned}
$$

A partir del anterior resultado y considerando (4.38) obtenemos finalmente

$$
\sum_{i=j+1}^{s} b_{i} \Phi_{i}(t)=\frac{1}{(p+2) \cdot(p+1) \cdot p \cdot c} .
$$

Como $a_{i j}=0$ siempre que $i \geq j$, el anterior sumatorio está extendido para los valores de $i$ para los que tiene sentido, entonces (4.35) nos permite afirmar que se satisface también la condición de orden para el árbol $w$. 
Nota 4.2.24 Como se puede ver en Hairer [53], para métodos con órdenes muy elevados el Lema 4.2.23 se puede generalizar imponiendo

$$
\sum_{i=j+1}^{s} b_{i} c_{i}^{k} a_{i j}=b_{j}\left(\frac{c_{j}^{k+2}}{(k+2)(k+1)}-\frac{c_{j}}{(k+1)}+\frac{1}{(k+2)}\right)
$$

para $j=1, \ldots, s-1$ y $k=1,2, \ldots$

\subsection{Un método $\mathrm{RKN} h^{2}$ de paso variable y orden 8}

A lo largo de esta sección seguiremos la construcción de un par encajado 8(6) a partir de dos fórmulas $\mathrm{RKN} h^{2}$ utilizándolas en modo de extrapolación local. De este modo avanzaremos con el esquema de orden 8 , utilizando el método de orden 6 para estimar el error local. Como ya hicimos en los pares 4(3) del capítulo anterior, buscaremos que el par obtenido alcance órdenes mayores cuando se trata de integrar un oscilador no perturbado. Comencemos obteniendo el método de orden 8. Emplearemos la misma filosofía y notación que en los pares 4(3) obtenidos previamente.

\subsubsection{Construcción del método de orden 8}

Aunque se pueden obtener métodos $\mathrm{RKN} h^{2}$ de orden 8 con $s=8$ etapas, no debemos perder de vista que perseguimos encajar esta fórmula con un método de orden menor para conseguir el par. Por ello, nosotros hemos considerado $s=9$ para conseguir que los dos métodos del par encajado fuesen lo más diferentes posible. Habitualmente en pares encajados RK y RKN, se construye el par utilizando la propiedad FSAL debida a Dormand y Prince (véase Dormand [19], Dormand et al. [20, 21], Hairer et al. [54] o Lambert [69]). Ésta consiste en añadir una etapa más sólo al método de orden bajo imponiendo que la última evaluación de cada paso sea la misma que la primera en el siguiente. Así, salvo en el primer paso, siempre calcularemos sólo $s$ evaluaciones de función, y no $s+1$, que podremos usar en ambas fórmulas. Es decir, para un método RKN de $s+1$ etapas se ha de 
cumplir

$$
\begin{aligned}
\left(k_{1}\right)_{n+1} & =f\left(x_{n+1}, \hat{y}_{n+1}\right)=f\left(x_{n}+h, \hat{y}_{n}+h \hat{y}_{n}^{\prime}+h^{2} \sum_{j=1}^{s+1} \hat{\bar{b}}_{j} k_{j}\right) \\
& =f\left(x_{n}+h c_{s+1}, \hat{y}_{n}+h c_{s+1} \hat{y}_{n}^{\prime}+h^{2} \sum_{j=1}^{s} a_{s+1, j} k_{j}\right)=\left(k_{s+1}\right)_{n} .
\end{aligned}
$$

Esto nos lleva a las siguientes condiciones para algunos coeficientes

$$
c_{s+1}=1, \quad \hat{\bar{b}}_{s+1}=0, \quad a_{s+1, j}=\hat{\bar{b}}_{j} \quad j=1, \ldots, s .
$$

Sin embargo, observando (4.39) la propiedad FSAL implicaría para los métodos RKNh $h^{2}$ lo siguiente

$$
\sum_{j=1}^{s} a_{s+1, j} k_{j}=\sum_{j=1}^{s+1}\left(\hat{\bar{b}}_{j}+h^{2} \omega^{2}{\hat{\overline{b^{*}}}}_{j}\right) k_{j}
$$

y entonces los coeficientes $a_{s+1, j}$ dependerían de $h^{2}$.

Buscamos por tanto un método $\mathrm{RKN}^{2}$ con $s=9$ etapas que alcance orden 8 al integrar numéricamente el problema (2.2). Impondremos $\hat{\bar{b}}_{9}=\hat{b}_{9}=0$. En un primer intento obtuvimos un método con orden oscilatorio 9, pero no proporcionaba resultados satisfactorios comparado con ciertos métodos RKN de orden 8 que mencionaremos más adelante. Este motivo nos llevó a buscar métodos con el mayor orden oscilatorio posible, que resultó ser 11. Para ello fue preciso resolver el conjunto de ecuaciones del Teorema 4.2 .11 con $p=8$ y $q=11$. Teniendo en cuenta las condiciones simplificadoras de los Lemas 4.2.13, $4.2 .15,4.2 .19,4.2 .20,4.2 .21,4.2 .22$ y 4.2 .23 los coeficientes buscados se obtienen resolviendo las siguientes ecuaciones:

$$
\begin{aligned}
\hat{\bar{b}}_{i} & =\hat{b}_{i}\left(1-c_{i}\right), \quad i=1, \ldots, 9, \\
\sum_{j=1}^{i-1} a_{i j} & =\frac{c_{i}^{2}}{2}, \quad i=2, \ldots, 9, \\
\sum_{j=1}^{i-1} a_{i j} c_{j} & =\frac{c_{i}^{3}}{6}, \quad i=3, \ldots, 9, \\
\sum_{j=1}^{i-1} a_{i j} c_{j}^{2} & =\frac{c_{i}^{4}}{12}, \quad i=3, \ldots, 9, \\
\sum_{i=j+1}^{9} \hat{b}_{i} a_{i j} & =\hat{b}_{j}\left(\frac{c_{j}^{2}}{2}-c_{j}+\frac{1}{2}\right), \quad j=1, \ldots, 8,
\end{aligned}
$$




$$
\begin{aligned}
& \sum_{i=1}^{9} \hat{b}_{i} c_{i}^{k}=\frac{1}{k+1}, \quad k=0, \ldots, 7, \\
& \sum_{i=3}^{9} \hat{b}_{i} c_{i}^{k} \sum_{j=2}^{i-1} a_{i j} c_{j}^{3}=\frac{1}{(k+6) \cdot 5 \cdot 4}, \quad k=0,1,2 \\
& \sum_{i=3}^{9} \hat{b}_{i} c_{i}^{k} \sum_{j=2}^{i-1} a_{i j} c_{j}^{4}=\frac{1}{(k+7) \cdot 6 \cdot 5}, \quad k=0,1 \\
& \sum_{i=3}^{9} \hat{b}_{i} c_{i}^{k} \sum_{j=2}^{i-1} a_{i j} c_{j}^{5}=\frac{1}{(k+8) \cdot 7 \cdot 6}, \quad k=0, \\
& \sum_{i=3}^{9} \hat{b}_{i} c_{i}^{k} a_{i 2}=0, \quad k=0,1,2, \\
& \sum_{i=1}^{9} \hat{\overline{b_{i}^{*}}} c_{i}^{k}=0, \quad k=0, \ldots, 4, \\
& \sum_{i=1}^{9} \hat{b_{i}^{*}} c_{i}^{k}=0, \quad k=0, \ldots, 5, \\
& \sum_{i=3}^{9} \hat{b_{i}^{*}} \sum_{j=2}^{i-1} a_{i j} c_{j}^{3}=0 \\
& \sum_{i=3}^{9} \hat{b_{i}^{*}} a_{i 2}=0 \\
& \sum_{i=4}^{9} \hat{\overline{b_{i}^{*}}} \sum_{j=3}^{i-1} a_{i j} c_{j}^{3}=\sum_{i=5}^{9} \hat{\bar{b}}_{i} \sum_{j=4}^{i-1} a_{i j} \sum_{k=3}^{j-1} a_{j k} c_{k}^{3}-\frac{1}{60480}, \\
& \sum_{i=4}^{9} \hat{b}_{i}^{*} \sum_{j=3}^{i-1} a_{i j} c_{j}^{4}=\sum_{i=5}^{9} \hat{b}_{i} \sum_{j=4}^{i-1} a_{i j} \sum_{k=3}^{j-1} a_{j k} c_{k}^{4}-\frac{1}{15120} \\
& \sum_{i=4}^{9} \hat{\hat{b}_{i}^{*}} \sum_{j=3}^{i-1} a_{i j} c_{j}^{4}=\sum_{i=5}^{9} \hat{\bar{b}}_{i} \sum_{j=4}^{i-1} a_{i j} \sum_{k=3}^{j-1} a_{j k} c_{k}^{4}-\frac{1}{151200} \\
& \sum_{i=5}^{9} \hat{b_{i}^{*}} \sum_{j=4}^{i-1} a_{i j} \sum_{k=3}^{j-1} a_{j k} c_{k}^{3}=\sum_{i=6}^{9} \hat{b}_{i} \sum_{j=5}^{i-1} a_{i j} \sum_{k=4}^{j-1} a_{j k} \sum_{l=3}^{k-1} a_{k l} c_{l}^{3}-\frac{1}{604800}, \\
& \sum_{i=5}^{9} \hat{\hat{b}_{i}^{*}} \sum_{j=4}^{i-1} a_{i j} \sum_{k=3}^{j-1} a_{j k} c_{k}^{3}=\sum_{i=6}^{9} \hat{\hat{b}_{i}} \sum_{j=5}^{i-1} a_{i j} \sum_{k=4}^{j-1} a_{j k} \sum_{l=3}^{k-1} a_{k l} c_{l}^{3}-\frac{1}{6652800}
\end{aligned}
$$




$$
\sum_{i=5}^{9} \hat{b_{i}^{*}} \sum_{j=4}^{i-1} a_{i j} \sum_{k=3}^{j-1} a_{j k} c_{k}^{4}=\sum_{i=6}^{9} \hat{b}_{i} \sum_{j=5}^{i-1} a_{i j} \sum_{k=4}^{j-1} a_{j k} \sum_{l=3}^{k-1} a_{k l} c_{l}^{4}-\frac{1}{1663200} .
$$

Las ecuaciones (4.40)-(4.44) son las condiciones simplificadoras utilizadas. Las ecuaciones (4.45)-(4.48) y (4.50)-(4.52) son las condiciones de orden 8 para los coeficientes $\hat{b}_{i}, \hat{\bar{b}}_{i}^{*}$ y $\hat{b}_{i}^{*}$ respectivamente. Por último, las ecuaciones (4.54)-(4.59) son las condiciones para alcanzar orden oscilatorio 11. Para poder aplicar las correspondientes condiciones simplificadoras y reducir así el número de árboles a considerar, es necesario que se cumplan (4.49) y (4.53). Por idéntica razón es preciso que $\hat{b}_{2}=\hat{\bar{b}}_{2}=\hat{b}_{2}^{*}=\hat{\bar{b}}_{2}^{*}=0$.

Si consideramos las ecuaciones (4.41), (4.42) y (4.43) para $i=3$ resulta un sistema lineal de tres ecuaciones para $a_{31}$ y $a_{32}$. Dicho sistema tiene solución no nula si

$$
c_{2}=\frac{c_{3}}{2}
$$

Esto nos proporciona una condición más que relaciona los coeficientes $c_{2}$ y $c_{3}$.

Las condiciones simplificadoras empleadas hacen que los árboles de Nyström considerados para obtener las ecuaciones para un método de orden 8 sean los de la Figura 4.10. Pero no todos ellos son independientes: los árboles correspondientes a las ecuaciones (4.46), (4.47) y (4.48) para $k=0$ ya están considerados en virtud del Lema 4.2.23, pero los incluiremos porque nos serán útiles a la hora de buscar los coeficientes del método siguiendo el trabajo debido a Hairer [53]. Las condiciones de orden oscilatorio 11 son las proporcionadas por los árboles $t^{8}, \ldots, t^{11}$ que se obtienen de forma sencilla como ya comentamos en la sección 4.2.5.

Pese a la enorme reducción del número de ecuaciones que ha tenido lugar tras la imposición de las condiciones simplificadoras, el intento por resolver de forma simbólica estas ecuaciones con Maple V 4 y empleando un ordenador Pentium III 500 con 128 Mb de RAM ejecutando Windows 2000 5.0 SP2 resultó fallido. Este hecho motivó la búsqueda de vías alternativas para la resolución de los correspondi- 


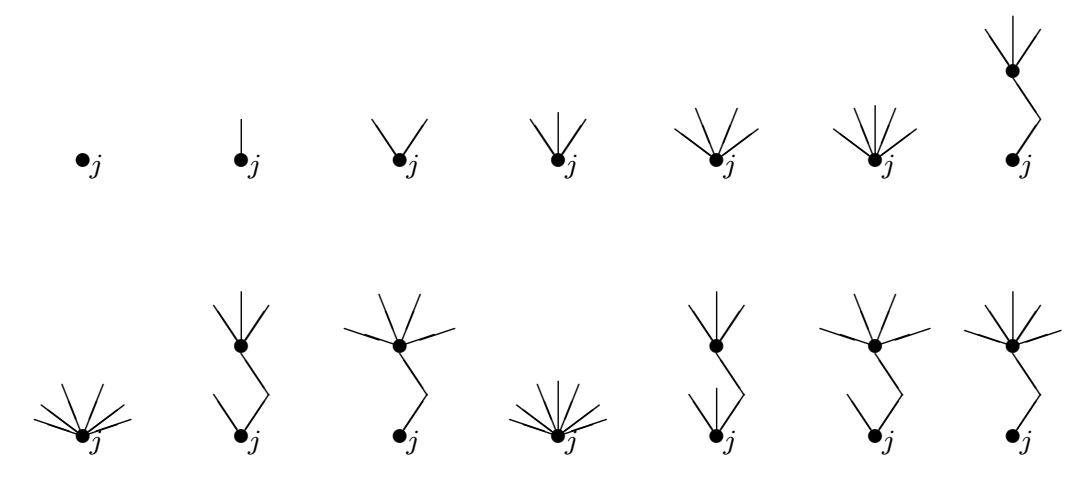

Figura 4.10: $S N T$ para un método de orden 8 .

entes sistemas de ecuaciones, como ya han hecho otros autores: Calvo [7], González [44], Hairer [52, 53],...

Tras diversos intentos nos decidimos a seguir los pasos del trabajo de Hairer [53] eligiendo de forma adecuada los coeficientes $c_{i}$. Así impusimos las siguientes condiciones:

$$
c_{1}=0, \quad c_{8}=1, \quad c_{3}=c_{7}, \quad \hat{b}_{3}+\hat{b}_{7}=0,
$$

y tomamos $c_{4}, c_{5}$ y $c_{6}$ como una de las permutaciones de los nodos de cuadratura de Lobatto De esta forma, $\hat{b}_{1}, \hat{b}_{4}, \hat{b}_{5}, \hat{b}_{6}$ y $\hat{b}_{8}$ son los correspondientes pesos que satisfacen

$$
\sum_{i=1}^{5} \omega_{i} \alpha_{i}^{k}=\frac{1}{k+1}, \quad k=0, \ldots, 4
$$

siendo $\alpha_{1}=0, \alpha_{2}=1 / 2-\sqrt{21} / 14, \alpha_{3}=1 / 2, \alpha_{4}=1 / 2+\sqrt{21} / 14, \alpha_{5}=$ 1 los mencionados nodos. Ciertos aspectos de cuadratura numérica pueden encontrarse en Stoer y Burlisch [104] y más en concreto en Gander y Hřebíček [42] cuyas rutinas numéricas ligeramente modificadas nos proporcionaron los nodos y pesos de la cuadratura de Lobatto con aritmética exacta. La elección de este tipo de nodos es la empleada en los mencionados trabajos de Hairer [52, 53] y González [44]. Sin embargo, siguiendo esta idea, la búsqueda de los parámetros 


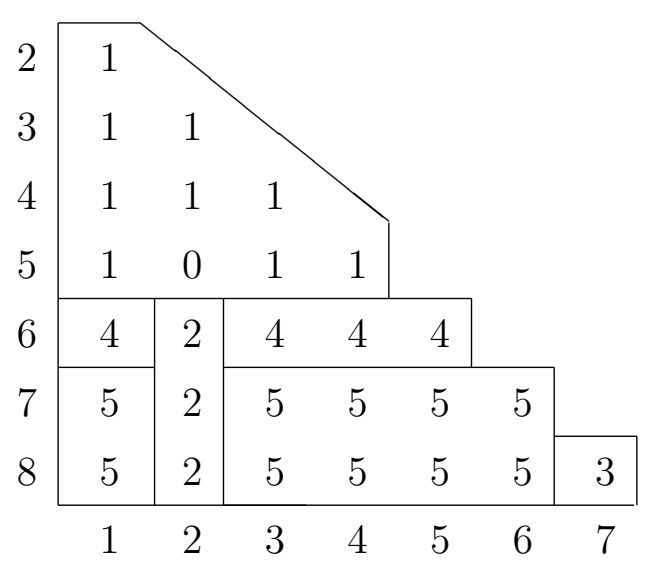

Figura 4.11: Pasos seguidos para la obtención de los $a_{i, j}$ hasta $i=8$.

libres desemboca en funciones que Maple V 4 no es capaz de minimizar. Finalmente nos decidimos por los $c_{i}$ del método RKN8(6) debido a Dormand et al. [21], esquema que presenta un buen comportamiento. Estos coeficientes son los que siguen:

$$
\begin{aligned}
& c_{1}=0, \quad c_{2}=\frac{1}{20}, \quad c_{3}=\frac{1}{10}, \quad c_{4}=\frac{3}{10}, \\
& c_{5}=\frac{1}{2}, \quad c_{6}=\frac{7}{10}, \quad c_{7}=\frac{9}{10}, \quad c_{8}=1, \quad c_{9}=1 .
\end{aligned}
$$

Nótese que $c_{2}$ y $c_{3}$ satisfacen la condición (4.60).

Fijados los coeficientes $c_{i}$, las ecuaciones (4.45) constituyen un sistema lineal en las variables $\hat{b}_{1}, \hat{b}_{3}, \ldots, \hat{b}_{8}$. Obtenidos los coeficientes $\hat{b}_{i}$, de forma inmediata (4.40) nos permite calcular los $\hat{\bar{b}}_{i}$. Obviamente todos ellos toman los mismos valores que los correspondientes a la fórmula de orden 8 del par debido a Dormand et al. [21].

Para obtener los coeficientes $a_{i j}$ con $i=2, \ldots, 8, j=1, \ldots, i-1$, seguiremos los siguientes pasos, que de forma esquemática se ilustran en la Figura 4.11.

Paso 1: A partir de la condición (4.41) para $i=2$, calculamos $a_{21}$. Con (4.41) y (4.42) para $i=3$, obtuvimos $a_{31}$ y $a_{32}$, y con (4.41), (4.42) y (4.43) para $i=4 \operatorname{los}$ coeficientes $a_{41}, a_{42}$ y $a_{43}$. Imponiendo 
$a_{52}=0$, de idéntica forma los ecuaciones $(4.41),(4.42)$ y (4.43) para $i=5$, nos proporcionan $a_{51}, a_{53}$ y $a_{54}$.

Paso 2: Empleando las ecuaciones (4.49) obtuvimos $a_{62}, a_{72}$ y $a_{82}$.

Paso 3: Gracias a la relación (4.44) para $j=7$ pudimos obtener $a_{87}$. Paso 4: Primero calculamos las expresiones

$$
\sum_{j=2}^{i-1} a_{i j} c_{j}^{3}
$$

para $i=2,3,4,5$, ya que todos los coeficientes han sido calculados en pasos anteriores. Posteriormente resolvimos el sistema (4.46) en las variables

$$
\begin{aligned}
& A_{1}=\sum_{j=2}^{i-1} a_{6 j} c_{j}^{3}, \\
& A_{2}=\sum_{j=2}^{i-1} a_{7 j} c_{j}^{3}, \\
& A_{3}=\sum_{j=2}^{i-1} a_{8 j} c_{j}^{3} .
\end{aligned}
$$

De forma similar a como hicimos en el paso $1, a_{61}, a_{63}, a_{64}$ y $a_{65}$ se obtuvieron a partir de las condiciones simplificadoras (4.41)-(4.43) añadiendo además (4.61).

Paso 5: Es similar al paso anterior. En este caso se calculan previamente las expresiones

$$
\sum_{j=2}^{i-1} a_{i j} c_{j}^{4}
$$

para $i=2,3,4,5,6$ y se resuelve el sistema formado por las ecuaciones (4.47) en las variables

$$
\begin{aligned}
& B_{1}=\sum_{j=2}^{i-1} a_{7 j} c_{j}^{4}, \\
& B_{2}=\sum_{j=2}^{i-1} a_{8 j} c_{j}^{4} .
\end{aligned}
$$

Con las ecuaciones (4.41)-(4.43) para $i=7$ junto con (4.62) y (4.64) obtuvimos los coeficientes $a_{71}, a_{73}, a_{74}, a_{75}$ y $a_{76}$. Análogamente a partir de (4.41)-(4.43) para $i=8$ añadiendo (4.63) y (4.65) calculamos los restantes coeficientes $a_{81}, a_{83}, a_{84}, a_{85}$ y $a_{86}$. 
Queda ahora comentar cómo hemos obtenido los coeficientes $\hat{b_{i}^{*}}$ y $\hat{\hat{b}_{i}^{*}} \operatorname{con} i=1, \ldots, 9 \mathrm{y} \operatorname{los} a_{9 j}$.

A partir de (4.51)-(4.53), (4.55), (4.57) y (4.59) se pudieron conseguir los valores de $\hat{b_{1}^{*}}$ y $\hat{b}_{i}^{*}$ para $i=3, \ldots, 8$ y $a_{9 j}$ para $j=2, \ldots, 5$. Resolviendo (4.52), (4.54), (4.56) y (4.58) calculamos los coeficientes $\hat{b_{1}^{*}}$ y $\hat{b_{i}^{*}}$ para $i=3, \ldots, 8$. Finalmente las condiciones simplificadoras (4.41)-(4.43) para $i=9$ nos proporcionaron los valores de $a_{91}, a_{96}$ y $a_{98}$.

De esta forma resolvimos todas las ecuaciones quedando libres las indeterminadas $a_{97}$ y $\hat{b_{9}^{*}}$, que posteriormente utilizamos para minimizar la norma euclídea de los términos de error correspondientes a las aproximaciones tanto a la solución, como a la derivada, con el fin de que el esquema de orden 8 fuese óptimo, tarea que llevamos a cabo de forma similar a como hicimos en el caso del método RKN $h^{2} 4: 5 M$ de la sección 2.4, utilizando la función extrema de Maple V 4.

Nota 4.3.1 En la búsqueda de los coeficientes del método de orden 8 se han utilizado gran parte de las ecuaciones de orden mostradas en el inicio de la sección, aunque no todas porque no consituyen un conjunto de ecuaciones independientes. Esto es debido, como ya hemos comentado, a que hemos añadido árboles redundantes con el fin de que la resolución de los sistemas fuese más sencilla, similar a los trabajos de Hairer [53]. Por otro lado, cuando $c_{i} \neq c_{j}$ si $i \neq j$ e $i=1, \ldots, 8$, se puede demostrar que las condiciones de orden (4.45)-(4.49) implican directamente (4.44). Así podríamos haber buscado el método de orden 8 empleando las funciones $Q_{i k}$ y $R_{k j}$ de los trabajos de Dormand et al. [20, 21].

\subsubsection{Construcción del método de orden 6}

Inicialmente tratamos de buscar un método de orden 7 con orden oscilatorio 8, pero los coeficientes obtenidos coincidían con los del método de orden alto. Este hecho nos llevó a considerar un esquema de orden 6. La búsqueda de este método fue una tarea más sencilla debido a que el número de ecuaciones era menor y gran parte de los coeficientes del método ya venían determinados por la fórmula de orden alto. Para encontrar métodos de orden 6 y orden oscilatorio 
7 es preciso que los coeficientes del método satisfagan las siguientes ecuaciones:

$$
\begin{aligned}
\sum_{i=1}^{9} b_{i} c_{i}^{k} & =\frac{1}{k+1}, \quad k=0, \ldots, 5, \\
\sum_{i=3}^{9} b_{i} \sum_{j=2}^{i-1} a_{i j} c_{j}^{3} & =\frac{1}{6 \cdot 5 \cdot 4}, \\
\sum_{i=1}^{9} \bar{b}_{i}^{*} c_{i}^{k} & =0, \quad k=0,1,2, \\
\sum_{i=1}^{9} b_{i}^{*} c_{i}^{k} & =0, \quad k=0, \ldots, 3, \\
\sum_{i=3}^{9} \bar{b}_{i}^{*} c_{i}^{3} & =\sum_{i=4}^{9} \hat{\bar{b}}_{i} \sum_{j=3}^{i-1} a_{i j} c_{j}^{3}-\frac{1}{840} \\
\sum_{i=3}^{9} b_{i}^{*} c_{i}^{4} & =\sum_{i=4}^{9} b_{i} \sum_{j=3}^{i-1} a_{i j} c_{j}^{4}-\frac{1}{210}
\end{aligned}
$$

correspondientes a los coeficientes $b_{i}, \bar{b}_{i}^{*}$ y $b_{i}^{*}$. Una vez calculados los $b_{i}$, los coeficientes $\bar{b}_{i}$ se encuentran de forma análoga al método de orden alto con las relaciones:

$$
\bar{b}_{i}=b_{i}\left(1-c_{i}\right), \quad i=1, \ldots, 9 .
$$

De nuevo las ecuaciones (4.66)-(4.69) son las correspondientes condiciones de orden 6 , y (4.70) y (4.71) las ecuaciones para alcanzar orden oscilatorio 7 . Resolviendo las ecuaciones anteriores resultaron 9 parámetros libres, que como ya hicimos para el método $\mathrm{RKN} h^{2} 4: 6(3: 4)$ desarrollado en el capítulo precedente, elegimos para que los correspondientes coeficientes $B^{(8)}, B^{\prime(8)}, C^{(7)}$ y $C^{\prime(7)}$ fuesen pequeños y así la estimación del error lo más fiel posible. Previamente fijamos ciertos valores de los parámetros libres de modo que no se cumpliesen las correspondientes ecuaciones de orden 7 y orden oscilatorio 8 y así el método de orden bajo tuviese el orden deseado. Los valores elegidos garantizaban además que los coeficientes $\bar{b}_{i}, b_{i}, \bar{b}_{i}^{*}$ y $b_{i}^{*}$ restantes estuviesen en el intervalo $[-1,1]$.

A continuación se incluyen los coeficientes del método obtenido que, siguiendo la notación establecida, llamaremos $\mathrm{RKN} h^{2} 8: 11(6: 7)$. 


$$
\begin{aligned}
& a_{2,1}=\frac{1}{800}, \quad a_{3,1}=\frac{1}{600}, \quad a_{3,2}=\frac{1}{300}, \\
& a_{4,1}=\frac{9}{200}, \quad a_{4,2}=\frac{-9}{100}, \quad a_{4,3}=\frac{9}{100}, \\
& a_{5,1}=\frac{1}{48}, \quad a_{5,2}=0, \quad a_{5,3}=\frac{5}{96}, \\
& a_{5,4}=\frac{5}{96}, \quad a_{6,1}=\frac{-56791}{222000}, \quad a_{6,2}=\frac{1666}{2775}, \\
& a_{6,3}=\frac{-6713}{29600}, \quad a_{6,4}=\frac{245}{3552}, \quad a_{6,5}=\frac{539}{9250}, \\
& a_{7,1}=\frac{127179}{164500}, \quad a_{7,2}=\frac{-7569}{4700}, \quad a_{7,3}=\frac{18303}{18800}, \\
& a_{7,4}=\frac{819}{3760}, \quad a_{7,5}=\frac{-108}{5875}, \quad a_{7,6}=\frac{114}{1645}, \\
& a_{8,1}=\frac{-52691}{21408}, \quad a_{8,2}=\frac{28325}{5352}, \quad a_{8,3}=\frac{-145695}{57088}, \\
& a_{8,4}=\frac{-805}{3568}, \quad a_{8,5}=\frac{13335}{28544}, \quad a_{8,6}=\frac{-705}{14272}, \\
& a_{8,7}=\frac{1645}{57088}, \quad a_{9,1}=\frac{994504107}{25000000}, \\
& a_{9,2}=\frac{-33212673736579434846689079566967852067}{1660899109075482077058488451189750000}, \\
& a_{9,3}=\frac{-70553478436066909868143867546115131611791947}{1657444438928605074338206795211275320000000}, \\
& a_{9,4}=\frac{3471068868153604904036771637389582336269}{179044923958336967906905055038255050000}, \\
& a_{9,5}=\frac{2670944043902080461381447103732604997741233}{153467077678574543920204332889932900000000}, \\
& a_{9,6}=\frac{-949664280542831457337540361787622800545249}{138120369910717089528183899600939610000000}, \\
& a_{9,7}=\frac{-43694959368739267015472991075414815984221}{1860207002164539926305507065332520000000}, \\
& a_{9,8}=\frac{6294421983065912825000000000}{373365757088517101462732871},
\end{aligned}
$$




$$
\begin{aligned}
& \hat{\bar{b}}_{1}=\frac{223}{7938}, \quad \hat{\bar{b}}_{2}=0, \quad \hat{\bar{b}}_{3}=\frac{1175}{8064}, \\
& \hat{\bar{b}}_{4}=\frac{925}{6048}, \quad \hat{\bar{b}}_{5}=\frac{41}{448}, \hat{\bar{b}}_{6}=\frac{925}{14112}, \\
& \hat{\bar{b}}_{7}=\frac{1175}{72576}, \hat{\bar{b}}_{8}=0, \quad \hat{\bar{b}}_{9}=0 \text {, } \\
& \hat{b}_{1}=\frac{223}{7938}, \quad \hat{b}_{2}=0, \quad \hat{b}_{3}=\frac{5875}{36288}, \\
& \hat{b}_{4}=\frac{4625}{21168}, \quad \hat{b}_{5}=\frac{41}{224}, \quad \hat{b}_{6}=\frac{4625}{21168}, \\
& \hat{b}_{7}=\frac{5875}{36288}, \hat{b}_{8}=\frac{223}{7938}, \quad \hat{b}_{9}=0 \text {, } \\
& \hat{b_{1}^{*}}=\frac{120517713150354725873809026321001395360437}{109972661365416627627100586542968750000000000}, \\
& \hat{b_{2}^{*}}=0 \text {, } \\
& \hat{b_{3}^{*}}=\frac{-46106911575464960046030898669085052853952717}{177363908250143937036987825976500000000000000000}, \\
& \hat{b_{4}^{*}}=\frac{10674703909260670639131044930710642617984239}{34487426604194654423858743939875000000000000000} \text {, } \\
& \hat{b_{5}^{*}}=\frac{-17941311880099063788755370915178802853952717}{821129204861777748628235104618750000000000000000} \text {, } \\
& \hat{b_{6}^{*}}=\frac{551216004630873665731086719746525407707531}{14780325687511994753082318831375000000000000000} \text {, } \\
& \hat{\overline{b_{7}^{*}}}=\frac{1136031979474092496502239648747494127338587}{1970710091668265967077642510850000000000000000}, \\
& \hat{b_{8}^{*}}=\frac{-17190153161813383124503313207109745796828533}{484979436621487327835513586654492187500000000000} \text {, } \\
& \hat{b_{9}^{*}}=\frac{-38937}{250000000000}, \\
& \hat{b_{1}^{*}}=\frac{-158141506376075320050497204938384646047283}{10038266444956224677912955524840625000000000000000}, \\
& \hat{b_{2}^{*}}=0 \text {, } \\
& \hat{b_{3}^{*}}=\frac{158141506376075320050497204938384646047283}{36711374444113359649388077656000000000000000000},
\end{aligned}
$$




$$
\begin{aligned}
& \hat{b_{4}^{*}}=\frac{-158141506376075320050497204938384646047283}{21414968425732793128809711966000000000000000000}, \\
& \hat{b_{5}^{*}}=\frac{158141506376075320050497204938384646047283}{16996006687089518356198184100000000000000000000}, \\
& \hat{b_{6}^{*}}=\frac{-158141506376075320050497204938384646047283}{21414968425732793128809711966000000000000000000}, \\
& \hat{b_{7}^{*}}=\frac{158141506376075320050497204938384646047283}{36711374444113359649388077656000000000000000000}, \\
& \hat{b_{8}^{*}}=\frac{-82606929081151911714771634844120796828533}{100382664495622467791295524840625000000000000000}, \\
& \hat{b_{9}^{*}}=\frac{-10421875559551203}{13850287844000000000000}, \\
& \bar{b}_{1}=\frac{1397094195674}{53806306640625}, \quad \bar{b}_{2}=0, \\
& \bar{b}_{3}=\frac{6600563561777}{43728300000000}, \quad \bar{b}_{4}=\frac{4787014563223}{32796225000000}, \\
& \bar{b}_{5}=\frac{1187958687259}{12146750000000}, \quad \bar{b}_{6}=\frac{4787014563223}{76524525000000}, \\
& \bar{b}_{7}=\frac{6600563561777}{393554700000000}, \quad \bar{b}_{8}=0 \text {, } \\
& \bar{b}_{9}=0, \quad b_{1}=\frac{1397094195674}{53806306640625}, \\
& b_{2}=0, \quad b_{3}=\frac{6600563561777}{39355470000000}, \\
& b_{4}=\frac{4787014563223}{22957357500000}, \quad b_{5}=\frac{1187958687259}{6073375000000}, \\
& b_{6}=\frac{4787014563223}{22957357500000}, \quad b_{7}=\frac{6600563561777}{39355470000000}, \\
& b_{8}=\frac{132021343833695039162708094251727321527425437987353}{4893047949788074911936883248078900129140001978515625}, \\
& b_{9}=\frac{-291547127602519717045485560625231427629}{286909978502885356388337367211387900103035}, \\
& \overline{b_{1}^{*}}=\frac{35525087}{600000000000}, \quad \overline{b_{2}^{*}}=0, \\
& \overline{b_{3}^{*}}=\frac{-399134801}{4000000000000}, \quad \overline{b_{4}^{*}}=\frac{537055727}{12000000000000},
\end{aligned}
$$




$$
\begin{gathered}
\overline{b_{5}^{*}}=\frac{-2089711}{2000000000000}, \quad \overline{b_{6}^{*}}=\frac{-2089711}{4000000000000}, \\
\overline{b_{7}^{*}}=\frac{-2089711}{4000000000000}, \quad \overline{b_{8}^{*}}=\frac{-2089711}{2000000000000}, \\
\overline{b_{9}^{*}}=\frac{-2089711}{2000000000000}, \\
b_{1}^{*}=\frac{-439812717071188382219965958478072428887174134539657993}{1721459871017312138330024203268327400618210000000000000}, \\
b_{2}^{*}=0, \\
b_{3}^{*}=\frac{1644895610209920851750144102090022430736356271063992759}{2754335793627699421328038725229323840989136000000000000}, \\
b_{4}^{*}=\frac{-1946469977391889261781846757465178924765077375948521437}{2754335793627699421328038725229323840989136000000000000}, \\
b_{5}^{*}=\frac{2489302974954561554208903720714890733362442403153416103}{4590559656046165702213397875382206401648560000000000000}, \\
b_{6}^{*}=\frac{-536558220976427323667426360780059868913439198483303423}{2754335793627699421328038725229323840989136000000000000}, \\
b_{7}^{*}=\frac{-2089711}{4000000000000}, \quad b_{8}^{*}=\frac{875991}{50000000}, \quad b_{9}^{*}=\frac{-2089711}{2000000000000} .
\end{gathered}
$$

\subsubsection{Experimentos numéricos.}

Con el propósito de estudiar el comportamiento del esquema de orden 8 construido en las secciones precedentes, tanto en paso fijo como variable, se han realizado diversos experimentos numéricos integrando varios de los problemas anteriormente considerados con distintos métodos del mismo orden y el par de orden 4 obtenido en el capítulo anterior.

Consideraremos, por tanto, dos problemas oscilatorios

1. El problema de Bessel (3.4), que es un test muy empleado para valorar la eficiencia de esquemas de paso variable, integrándolo en los intervalos $[1,10],[0.1,10]$ y $[0.01,10]$.

2. El problema del satélite artificial ecuatorial (2.69) integrando una órbita circular y otra altamente excéntrica.

La integración numérica de dichos problemas se ha llevado a cabo con los siguientes esquemas: 
- El par encajado RKN8(6) con nueve etapas y verificando la propiedad FSAL (que hace que el método requiera ocho evaluaciones por paso) presentado Dormand et al. [21].

- El esquema de paso variable debido a González [44] y González et al. [47], que es un par de órdenes ocho y siete que requiere diez evaluaciones de función por paso. Este esquema ha sido especialmente desarrollado para integrar problemas oscilatorios y tiene orden oscilatorio infinito. Nos referiremos a este código como RKGM8(7).

- Los métodos desarrollados a lo largo del presente capítulo implementados tanto en paso fijo como en paso variable. En ambos casos se necesitan nueve evaluciones de función. Siguiendo la notación introducida nos referiremos a ellos como $\mathrm{RKN} h^{2} 8: 11$ para el método de paso fijo y $\mathrm{RKN} h^{2} 8: 11(6: 7)$ para el par encajado.

De nuevo hemos considerado una práctica muy común para estudiar la eficiencia de un método numérico, midiendo el coste computacional del método mediante el logaritmo decimal de las evaluaciones de función necesarias en toda la integración frente al logaritmo decimal del máximo error cometido a lo largo misma. Presentaremos en algunos casos, gráficas en las que se representa el coste computacional medido en tiempo de CPU. Todas las integraciones se realizaron en cuádruple precisión. Para los algoritmos en paso variable se ha considerado un tamaño de paso inicial de 0.1. Para los algoritmos de paso variable el tamaño de paso mínimo se ha tomado $10^{-5}$ para el primer problema en todos los métodos e intervalos, y $10^{-3}$ para el segundo problema en todos los esquemas, salvo en el de menor orden para el que se considera $10^{-5}$. No obstante, la integración en la mayoría de los casos se realiza con pasos alejados del tamaño mínimo. El tamaño de paso máximo permitido en todos los casos fue de 1.5.

Los distintos algoritmos se han implementado modificando convenientemente el programa DOPRIN que puede encontrarse en Hairer et al. [54] en la edición de 1986.

La Figura 4.12 corresponde a la integración del problema de Bessel en el intervalo $[1,10]$. Las tolerancias empleadas varían entre $10^{-2}$ y $10^{-15}$ para el método $\operatorname{RKN} 8(6), 10^{-3}$ y $10^{-16}$ para el esquema RKGM8(7), $10^{-2}$ y $10^{-10}$ para el algoritmo $R K N h^{2} 4: 6(3: 4)$ y $10^{-3}$ 
y $10^{-13}$ para el par $\mathrm{RKN} h^{2} 8: 11(6: 7)$. Para el método de paso fijo $\mathrm{RKN} h^{2} 8: 11$ se han considerado tamaños de paso que oscilan entre $1 / 2^{2}$ y $1 / 2^{7}$. Como era de esperar, los métodos de orden alto se muestran tanto más eficientes cuanto mayor sea la precisión requerida. De entre ellos los métodos RKN $h^{2}$ son los que presentan el mejor comportamiento, siendo mínima la diferencia entre el de paso fijo y el de paso variable. Estos, comparados con el método clásico y el método RKGM8(7), proporcionan resultados mejores. La Figura 4.13 pone de manifiesto que considerando el coste computacional en tiempo de CPU, la eficiencia del método RKN $h^{2} 8: 11(6: 7)$ es muy superior al esquema RKGM8(7), que ha sido desarrollado para integrar este tipo de problemas a costa de un importante gasto computacional. Frente al método RKN8(6) también presenta un ahorro en tiempo de CPU.

Figura 4.12: Problema de Bessel. $x \in[1,10]$

En las Figuras 4.14 y 4.15 se muestran los resultados de integrar el problema anterior en el intervalo $[0.1,10]$. En este caso se han considerado tolerancias entre $10^{-3}$ y $10^{-14}$ para el par RKN $h^{2} 8: 11(6: 7)$ y tamaños de paso entre $1 / 2^{2}$ y $1 / 2^{8}$ para el esquema $R K N h^{2} 8: 11$. En 
Figura 4.13: Problema de Bessel. $x \in[1,10]$

el resto de algoritmos se han empleado los mismos valores que en los intervalos anteriores. Podemos apreciar que, a pesar del excelente comportamiento que presentaba el método de paso fijo en el intervalo $[1,10]$, en este intervalo el método $\mathrm{RKN} h^{2} 8: 11$ ha empeorado, aunque sigue siendo más eficiente que el método RKN8(6). Esto es debido a que el extremo inferior del intervalo se encuentra más cercano a 0 , donde el problema de Bessel presenta serios problemas de integración. El método $\mathrm{RKN} h^{2} 8: 11(6: 7)$ sigue presentando los mejores resultados y, como se aprecia en la Figura 4.15, considerando el tiempo de CPU empleado en la integración, la ventaja frente al método RKGM8(7) es clara.

Las Figuras 4.16 y 4.17 corresponden a la integración del problema que nos ocupa en el intervalo [0.01,10]. Se han empleado los mismos valores que en el intervalo anterior, salvo en el esquema $\mathrm{RKN} h^{2} 4: 6(3: 4)$ para el que se han usado tolerancias entre $10^{-3}$ y $10^{-11}$. En este caso se aprecia claramente el pésimo comportamiento del método de paso fijo debido, como ya comentábamos para el caso anterior, a que la 
Figura 4.14: Problema de Bessel. $x \in[0.1,10]$

integración se inicia muy próxima a 0 . No obstante, como en los casos anteriores, el método $\mathrm{RKN} h^{2} 8: 11(6: 7)$ es el que se muestra más eficiente. En este intervalo, la diferencia con el esquema RKGM8(7) se hace menor aunque en tiempo de CPU, como podemos apreciar en la Figura 4.17, la eficiencia sigue siendo muy superior.

Para finalizar esta sección integraremos el problema del satélite artificial, que como ya hemos mencionado, no pretende ser un fiel test de eficiencia tanto como una aplicación del tipo de esquemas que hemos desarrollado a lo largo de la memoria.

En la Figuras 4.18 y 4.19 se presentan las gráficas de eficiencia correspondientes a la integración de 10 revoluciones para un satélite ecuatorial con excentricidades $e=0$ y $e=0.99$ respectivamente. Los valores de tolerancias empleados en ambos casos oscilan entre $10^{-6}$ y $10^{-17}$ para el método RKN8(6), $10^{-6}$ y $10^{-17}$ para el esquema RKGM8(7), $10^{-2}$ y $10^{-12}$ para el algoritmo RKNh $h^{2} 4: 6(3: 4)$ y $10^{-7}$ y $10^{-16}$ para el par $\mathrm{RKN} h^{2} 8: 11(6: 7)$. Para el método de paso fijo $\mathrm{RKN} h^{2} 8: 11$ se han considerado tamaños entre $1 / 2$ y $1 / 2^{4}$. De nuevo, 
Figura 4.15: Problema de Bessel. $x \in[0.1,10]$

los métodos RKNh $h^{2}$ manifiestan un excelente comportamiento. Como cabía esperar, el algoritmo en paso fijo proporciona unos resultados ligeramente superiores a los correspondientes para el esquema en paso variable. Esto puede ser debido a que el problema del satélite considerado es bastante regular y la integración en paso fijo con un método adecuado puede resultar suficiente.

Como conclusiones de los experimentos realizados podemos decir lo siguiente:

- Los métodos RKN $h^{2}$ de orden alto, implementados tanto en paso variable como en paso fijo, son más eficientes que los de orden bajo cuando se busca precisión elevada.

- Los esquemas RKNh $h^{2} 8: 11(6: 7)$ y RKNh $h^{2} 8: 11$ muestran una gran eficiencia comparados no sólo con un método clásico de propósito general como el algoritmo RKN8(6), sino también frente a los métodos RKGM desarrollados para integrar eficientemente problemas oscilatorios. 
Figura 4.16: Problema de Bessel. $x \in[0.01,10]$

- Los esquemas $\mathrm{RKN} h^{2}$ de paso fijo, tanto para orden alto como para órdenes menores (como pudimos ver en los experimentos del capítulo precedente) tienen un comportamiento excelente aún comparándolos con las correspondientes implementaciones en paso variable. Sin embargo, cuando la dificultad del problema así lo requiere, los métodos de paso variable proporcionan aproximaciones mucho más precisas.

- Los métodos RKNh $h^{2}$, además de mostrarse más precisos que los métodos RKGM, requieren un gasto computacional en tiempo de CPU comparable al de los esquemas clásicos. Esto se debe a la sencilla dependencia de sus coeficientes del tamaño de paso y al menor tiempo invertido en recalcularlos cuando la amplitud de paso cambia.

- Los métodos RKNh $h^{2}$ implementados en paso variable, se muestran como los algoritmos más eficientes de todos los comparados. 
Figura 4.17: Problema de Bessel. $x \in[0.01,10]$ 
Figura 4.18: Problema del satélite. $e=0$. 
Figura 4.19: Problema del satélite. $e=0.99$. 


\section{Bibliografía}

[1] Bettis, D.G., Numerical integration of products of Fourier and ordinary polinomials. Numer. Math. 14, 421-434 (1970).

[2] Bettis, D.G., Stabilization of finite difference methods of numerical integration. Celest. Mech. 2, 282-295 (1970).

[3] Bettis, D.G., A Runge-Kutta-Nyström algorithm. Celest. Mech. \& Dyn. Astron. 8, 229-233 (1973).

[4] Brock, P. y Murray, F.J., The use of exponential sums in stepby-step integration. M.T.A.C. 6, 138-150 (1952).

[5] Butcher, J.C., Coefficients for the study of Runge-Kutta integration processes. J. Aust. Math. Soc. 3, 185-201 (1963).

[6] Butcher, J.C., The Numerical Analysis of Ordinary Differential Equations. John Wiley \& Sons, Chichester (1987).

[7] Calvo, M.P., Métodos Runge-Kutta-Nyström simplécticos. Secretariado de Publicaciones e Intercambio Científico. Universidad de Valladolid, Valladolid (1992).

[8] Calvo, M.P. y Sanz-Serna, J.M, High-order symplectic RungeKutta-Nyström methods. SIAM J. Sci. Comput. 14, 936-952 (1992).

[9] Calvo, M.P. y Sanz-Serna, J.M., Order conditions for canonical Runge-Kutta-Nyström methods. BIT 32, 131-142 (1992).

[10] Cash, J.R., High order P-stable formulae for the numerical integration of periodic initial value problems. Numer. Math. 37, 355-370 (1981). 
[11] Cash, J.R., Efficient P-stable methods for periodic initial value problems. BIT 24, 248-252 (1984).

[12] Chan, R.P.K. y Murua, A., Extrapolation of symplectic methods for Hamiltonian problems. Appl. Numer. Math. 34, 189-205 (2000).

[13] Chawla, M.M., Two-step fourth order P-stable methods for second order differential equations. BIT 21, 190-193 (1981).

[14] Collatz, L., The Numerical Treatment of Differential Equations. Springer, Berlin (1966).

[15] Coleman, J.P., Characterisation of a class of P-stable methods for differential equations of second order. J. Comput. Appl. Math. 22, 137-141 (1988).

[16] Coleman, J.P. e Ixaru, L.Gr., P-stability and exponential-fitting methods for $y^{\prime \prime}=f(x, y)$. IMA J. Numer. Anal. 16, 179-199 (1996).

[17] Costabile, F. y Costabile, C., Two-step fourth-order P-stable methods for second order differential equations. BIT 22, 384386 (1982).

[18] De Meyer, H., Vanthournout, J., y Vanden Berghe, G., On a new type of mixed interpolation. J. Comput. Appl. Math. 30, 55-69 (1990).

[19] Dormand, J.R., Numerical Methods for Diferencial Equations. A computational Approach. CRC Press, Boca Ratón (1996).

[20] Dormand, J.R., El-Mikkawy, M.E.A. y Prince, P.J., Families of Runge-Kutta-Nyström formulae. IMA J. Numer. Anal. 7, 235250 (1987).

[21] Dormand, J.R., El-Mikkawy, M.E.A. y Prince, P.J., Highorder embedded Runge-Kutta-Nyström formulae. IMA J. Numer. Anal. 7, 423-430 (1987). 
[22] Fairén, V., Martín, P. y Ferrándiz, J.M., Numerical tracking of small deviations from analitically known periodic orbits. Computers in Physics 8, 455-461 (1994).

[23] Falkner, V.M., A Method of Numerical Solution of Differential Equations. Phil. Mag. S. 7, Vol. 21, N. 141, 624-640 (1936).

[24] Farto, J.M., González, A.B. y Martín, P., An algorithm for the systematic construction of solutions to perturbed problems. Comput. Phys. Commun. 111, 110-132 (1998).

[25] Farto, J.M. y Martín, P., A systematic approach to the integration of perturbed problems. Actes des IV èmes Journées Zaragoza-Pau de Mathématiques Appliquées, Publications de l'Université de Pau et des Pays de l'Adour, 169-176 (1995).

[26] Fatunla, S.O., Ikhile, M.N.O. y Otunta, F.O., A class of P-stable linear multistep numerical methods. Int. J. Comput. Math. 72, 1-13 (1999).

[27] Fehlberg, E., Classical seventh, sixth and fifth order RungeKutta-Nyström formulas with step-size control for general second order differential systems. NASA Technical Report 315 (1974).

[28] Ferrándiz, J.M., A new set of canonical variables for orbit calculation. ESA SP-255, 361-364, Darmstadt (1986).

[29] Ferrándiz, J.M., A general canonical transformation increasing the number of variables with application to the two-body problem. Celest. Mech. \& Dyn. Astron. 41, 345-357 (1988).

[30] Ferrándiz, J.M. y Martín, P., Acondicionamiento de problemas para la integración numérica. Aplicación al oscilador de Morse. Actas de las XIV Jornadas Hispano-Lusas de Matemáticas. Vol. 3, 1213-1217 (1989).

[31] Ferrándiz, J.M. y Martín, P., Special algorithms for the numerical integration of problems in orbital dynamics. Proceedings of the First International Colloquium on Numerical Analysis. D. Bainov and V. Covachev, eds., VSP, Zeist, 51-60 (1993). 
[32] Ferrándiz, J.M., Martín, P. y Vigo, J., Special algorithms to limit the error growth in long-term computation of satellite orbits. Advances in the Astronautical Sciences 79, 1167-1183 (1992).

[33] Ferrándiz, J.M. y Novo, S., Improved Bettis method for longterm prediction. Predictability, Stability and Chaos in N-Body Dynamical Systems, A.E. Roy Ed., Plenum Publishing Corporation, NATO ASI Series B, Vol. 272, 515-522 (1991).

[34] Ferrándiz, J.M., Sansaturio, M.E. y Pojman, J.R., Increased accuracy of computations in the main satellite problem through linearization methods. Celest. Mech. \& Dyn. Astron. 53, 347363 (1992).

[35] Ferrándiz, J.M., Vigo, J. y Martín, P., Reducing the error growth in the numerical propagation of satellite orbits. ESA SP-326, 4954, Darmstadt (1991).

[36] Fine, J.M., Interpolants for Runge-Kutta-Nyström methods. Computing 39, 27-42 (1987).

[37] Franco, J.M, Correas, F. y Pétriz, F., Métodos adaptados de tipo Störmer-Cowell de orden elevado. Rev. Internac. Métod. Numér. Cálc. Diseñ. Ingr. 7, 193-216 (1991).

[38] Franco, J.M. y Palacios, M., High order P-stable multistep methods. J. Comput. Appl. Math. 30, 1-10 (1990).

[39] García, A., Martín, P. y González, A.B., New methods for oscillatory problems based on classical codes. Appl. Numer. Math. (en prensa).

[40] García, A., Velasco, A. y Martín, P., Reducing the error growth in the numerical integration of oscillatory problems. Int. J. Appl. Sci. Comput. 6, 114-119 (1999).

[41] Gautschi, W., Numerical integration of ordinary differential equations based on trigonometric polynomials. Numer. Math. 3, 381-397 (1961). 
[42] Gander, W. y Hřebíček, J., Solving Problems in Scientific Computing Using Maple and Matlab. Springer, Berlin (1997).

[43] Gladwell, I., Shampine, L.F. y Brankin, R.W., Automatic selection of the initial step size for an ODE solver. J. Comput. Appl. Math. 18, 175-192 (1987).

[44] González, A.B., Métodos numéricos tipo Runge-Kutta para la integración de osciladores perturbados. Secretariado de Publicaciones e Intercambio Científico. Universidad de Valladolid. Valladolid (1999).

[45] González, A.B., Farto, J.M. y López, D.L., Reformulation of the RKGM methods using Scheifele expansions. Appl. Math. Lett. 13, 63-66 (2000).

[46] González, A.B. y Martín, P., A note concerning Gauss-Jackson method. Extracta Math. 11, 255-260 (1996).

[47] González, A.B., Martín, P. y Farto, J.M.: A new family of Runge-Kutta type methods for the numerical integration of perturbed oscillators. Numer. Math. 82, 635-646 (1999).

[48] González, A.B., Martín, P. y López, D.J., Embedded RungeKutta type methods based on the Scheifele $G$-functions. Proceedings of the $2^{\text {nd }}$ Meeting on Numerical Methods for Differential Equations. Coimbra, 67-73 (1998),

[49] González, A.B., Martín P. y López, D.J., Behaviour of a new type of Runge-Kutta methods when integrating satellite orbits. Celest. Mech. \& Dyn. Astron. 75, 29-38 (1999)

[50] González, A.B., Martín, P. y López, D.J., On the numerical integration of orbital problems with high order Runge-KuttaNyström methods. Appl. Numer. Math. 35, 1-10 (2000).

[51] Graf, O.F., Multirevolution methods for orbit integration. Lecture Notes in Mathematics 362, 471-490, Springer, Berlin (1973).

[52] Hairer, E., Méthodes de Nyström pour l'équation différentielle $y^{\prime \prime}=f(x, y)$. Numer. Math. 27 283-300 (1977). 
[53] Hairer, E., A one-step method of order 10 for $\ddot{y}=f(x, y)$. IMA J. Numer. Anal. 2, 83-94 (1982).

[54] Hairer, E. , Nørsett, S. P. y Wanner, G., Solving Ordinary Differential Equations I, Nonstiff Problems. Springer, Berlín (1993).

[55] Hairer, E. y Wanner, G., A theory for Nyström methods. Numer. Math. 25, 383-400 (1975).

[56] Hardy, D.J., Okunbor, D.I. y Skeel, R.D., Symplectic variable step size integration for $N$-body problems. Appl. Numer. Math. 29, 19-30 (1999).

[57] Henrici, P., Discrete Variable Methods in Ordinary Differential Equations. John Wiley \& Sons, New York (1962).

[58] Herrick, S., Astrodynamics. Vol. 2: Orbit Correction, Perturbation Theory Integration. Van Nostrand Reinhold, London (1972).

[59] van der Houwen, P.J. y Sommeijer, B.P., Linear multistep methods with reduced truncation error for periodic initial-value problems. IMA J. Numer. Anal. 4, 479-489 (1984).

[60] van der Houwen, P.J. y Sommeijer, B.P., Predictor-corrector methods for periodic second-order initial-value problems. IMA J. Numer. Anal. 7, 407-422 (1987).

[61] van der Houwen, P.J y Sommeijer, B.P., Explicit Runge-Kutta (-Nyström) methods wtih reduced phase errors for computing oscillating solutions. SIAM J. Numer. Anal. 24, 595-617 (1987).

[62] van der Houwen, P.J. y Sommeijer, B.P., Diagonally implicit Runge-Kutta-Nyström methods for oscillatory problems. SIAM J. Numer. Anal. 26, 414-429 (1989).

[63] Ixaru, L.Gr. y Paternoster, B., A conditionally P-stable fourthorder exponential-fitting method for $y^{\prime \prime}=f(x, y)$. J. Comput. Appl. Math. 106, 87-98 (1999).

[64] Jackson, J., Note on the numerical integration of $d^{2} x / d t^{2}=$ $f(x, t)$. Monthly Notices of the R. Astr. Soc. 84, 602-612 (1924). 
[65] Jain, M.K., Jain, U. y Anantha Krishnaiah, U., P-stable methods for periodic second order differential equations. BIT 19, 347355 (1979).

[66] Janin, G., Mission analysis for terrestrial satellites and planetary orbiters: software design and algorithm description. ESA STM208, Darmstadt (1979).

[67] Kirchgraber, U., An ODE-solver based on the method of averaging. Numer. Math. 53, 621-652 (1988).

[68] Kryloff, N. y Bogoliuboff, N., Introduction to Non-Linear Mechanics. Princeton University Press, Princeton (1947).

[69] Lambert, J.D., Numerical Methods for Ordinary Differential Systems. John Wiley 6 Sons, Chichester (1991).

[70] Lambert, J.D. y Watson, I.A., Symmetric multistep methods for periodic initial value problems. Journal of the Institute of Mathematics and Its Applications 18, 189-202 (1976).

[71] Li, S., Order properties and construction of symplectic RungeKutta methods. J. Comput. Math. 18, 645-656 (2000).

[72] López, D.J., Métodos Multipaso para la Integración Numérica de Problemas Lineales Perturbados. Secretariado de Publicaciones e Intercambio científico. Universidad de Valladolid. Valladolid (1999).

[73] López, D.J. y Martín, P., A numerical method for the integration of perturbed linear problems. Appl. Math. Comput. 96, 65-73 (1998).

[74] López, D., Martín, P. y Farto, J.M., Generalization of the Störmer method for perturbed oscillators without explicit first derivatives. J. Comput. Appl. Math. 111, 123-132 (1999).

[75] López, D.J., Martín, P. y García, A., A variable-stepsize variable-order multistep method for the integration of perturbed linear problems. Appl. Numer. Math. (en prensa). 
[76] Marchal, C., The three-body problem. Elsevier, Amsterdam (1990).

[77] Martín, P., Extensiones del Método de Scheifele para la Integración Numérica de Osciladores y Sistemas Lineales Perturbados. Secretariado de Publicaciones e Intercambio científico. Universidad de Valladolid. Valladolid (1995).

[78] Martín, P. y Farto, J.M., Increasing the order of the SMF method for a special type of problem. SIAM J. Numer. Anal. 35, 773-777 (1998).

[79] Martín, P. y Farto, J.M., Improved numerical integration of perturbed oscillators via average. Appl. Math. Comput. 99, 129139 (1999).

[80] Martín, P. y Ferrándiz, J.M., Relative behaviour of special algorithms for the numerical integration of satellite orbits. Advances in the Astronautical Sciences 82, 765-782 (1993).

[81] Martín, P. y Ferrándiz, J.M., Behaviour of the SMF method for the numerical integration of satellite orbits. Celest. Mech. $\&$ Dyn. Astron. 63, 29-40 (1995).

[82] Martín, P. y Ferrándiz, J.M., Multistep numerical methods based on the Scheifele $G$-functions with application to satellite dynamics. SIAM J. Numer. Anal. 34, 359-375 (1997).

[83] Martín, P. y Ferrándiz, J.M., Numerical integration of perturbed linear systems. Appl. Numer. Math. 31, 183-189 (1999).

[84] Martín, P., García, A. y López, D.J, Modified Taylor approximation of functions with periodic behaviour. J. Comput. Appl. Math. 130, 91-97 (2001).

[85] Martín, P., López, D.J. y García, A., Implementation of Falkner method for problems of the form $y^{\prime \prime}=f(x, y)$. Appl. Math. Comput. 109, 183-187 (2000).

[86] Martín, P. y Velasco, A., Numerical calculation of the frequency of an oscillatory problem. Appl. Math. Lett. 13, 91-96 (2000). 
[87] Melendo, B. y Palacios, M., A new approach to the construction of multirevolution methods and their implementation. Appl. Numer. Math. 23, 259-274 (1997).

[88] Melendo, B. y Palacios, M., Algorithms of multirevolution type for the large rate integration of almost periodic problems. Actas del XV CEDYA/V CMA, Volumen I, 23-26, Servicio de Publicacións da Universidade de Vigo (1998).

[89] Merson, R.H., Numerical integration of the differential equations of celestial mechanics. Royal Aircraft Establishment Technical Report 74184 (1974).

[90] Neta, B. y Ford, C.H., Families of methods for ordinary differential equations based on trigonometric polinomials. Celest. Mech. \&6 Dyn. Astron. 63, 29-40 (1984).

[91] Novo, S. y Rojo, J., Some remarks on an ODE-solver of Kirchgraber. Numer. Math. 61, 261-264 (1992).

[92] Novo, S. y Rojo, J., A long-term integrator based on Kirchgraber's LISP code. Proceedings of International Conference on Differential Equations (EQUADIFF 91), World Scientific, Singapore, 790-794 (1993).

[93] Ozawa, K., A four-stage implicit Runge-Kutta-Nyström method with variable coefficients for solving periodic initial value problems. Japan J. Indust. Appl. Math. 16, 25-46 (1999).

[94] Paternoster, B., Runge-Kutta(-Nyström) methods for ODEs with periodics solutions based on trigonometric polynomials. Appl. Numer. Math. 28, 401-412 (1998).

[95] Petzold, L., An efficient numerical method for highly oscillatory ordinary differential equations. SIAM J. Numer. Anal. 18, 455479 (1981).

[96] Poincaré, H., Les Méthodes Nouvelles de la Mécanique Céleste, tome II. Gauthier-Villars, Paris 1893. Reimpresión: Blanchard, Paris 1987. 
[97] Sanz-Serna, J.M. y Calvo, M.P, Numerical Hamiltonian Problems. Chapman \& Hall, London (1994).

[98] Scheifele, G., On numerical integration of perturbed linear oscillating systems. ZAMP 22, 186-210 (1971).

[99] Sharp, P.W. y Fine, J.M., Some Nyström pairs for the general 2nd order initial value problem. J. Comput. Appl. Math. 42, 279-291 (1992).

[100] Sharp, P.W. y Fine, J.M., A contrast of direct and transformed Nyström pairs. J. Comput. Appl. Math. 42, 293-308 (1992).

[101] Sheffield, C., Generalized multi-step methods with an application to orbit computation. Celest. Mech. 1, 46-58 (1969).

[102] Stiefel, E.L. y Bettis, D.G., Stabilization of Cowell's method. Numer. Math. 13, 154-175 (1969).

[103] Stiefel, E.L. y Scheifele, G., Linear and Regular Celestial Mechanics. Springer, New York (1971).

[104] Stoer, J. y Burlirsch, R., Introduction to Numerical Analysis. Springer, New York (1983).

[105] Sun, G., A simple way constructing symplectic Runge-Kutta methods. J. Comput. Math. 18, 61-68 (2000).

[106] Szebehely, V.G., Theory of Orbits. Academic Press, New York (1967).

[107] Van Daele, M., Vanden Berghe, G. y De Meyer, H., Properties and implementation of r-Adams methods based on mixed-type interpolation. Computers Math. Applic. 30, 37-54 (1995).

[108] Van Daele, M., Vanden Berghe, G., De Meyer, H. e Ixaru, L.Gr., Exponential-fitted four-step methods for $y^{\prime \prime}=f(x, y)$. Int. J. Comput. Math. 66, 299-309 (1998).

[109] Vanden Berghe, G., De Meyer, H., Van Daele, M. y Van Hecke, T., Exponentially-fitted explicit Runge-Kutta methods. Comput. Phys. Commun. 123, 7-15 (1999). 
[110] Vanden Berghe, G., De Meyer, H., Van Daele, M. y Van Hecke, T., Exponentially fitted Runge-Kutta methods. J. Comput. Appl. Math. 125, 107-115 (2000).

[111] Vanthournout, J., De Meyer, H. y Vanden Berghe, G., Multistep methods for ordinary differential equations based on algebraic and first order trigonometric polynomials. Computational Ordinary Differential Equations, pp. 61-72, Cash, J.R. y Gladwell, I. (Eds.). Clarendon Press, Oxford (1992).

[112] Vanthournout, J., Vanden Berghe, G. y De Meyer, H., Families of backward differentiation methods based on a new type of mixed interpolation. Computers Math. Applic. 20, 19-30 (1990).

[113] Verhulst, F., Nonlinear Differential Equations and Dynamical Systems. Springer, New York (1990).

[114] Vigo-Aguiar, J. y Ferrándiz, J.M., A general procedure for the adaptation of multistep algorithms to the integration of oscillatory problems. SIAM J. Numer. Anal. 35, 1684-1708 (1998). 